ASC Report No. 04/2012

Indefinite Hamiltonian systems whose Titchmarsh-Weyl coefficients have no finite generalized poles of non-negativity type

M. Langer, H. Woracek 


\section{Most recent ASC Reports}

03/2012 M. Aurada, M. Feischl, J. Kemetmüller, M. Page, D. Praetorius

Adaptive FEM with inhomogeneous dirichlet data: Convergence and quasioptimality in Rd

02/2012 P. Goldenits, G. Hrkac, D. Praetorius, D. Suess

An Effective Integrator for the Landau-Lifshitz-Gilbert Equation

01/2012 A. Arnold, L. Neumann, W. Hochhauser

Stability of glued and embedded Glass Panes: Dunkerley straight Line as a conservative Estimate of superimposed buckling Coefficients

43/2011 G. Hastermann, P. Lima, L. Morgado, E. Weinmüller

Numerical Solution of the Density Profile Equation with p-Laplacians

42/2011 M. Aurada, J.M. Melenk, D. Praetorius

Mixed Conforming elements for the large-body limit in micromagnetics: a finite element approach

41/2011 P. Amodio, T. Levitina, G. Settanni, E.B. Weinmüller

On the Calculation of the Finite Hankel Transform Eigenfunctions

40/2011 D.P. Hewett, S. Langdon, J.M. Melenk

A high frequency hp boundary element method for scattering by convex polygons

39/2011 A. Jüngel

Semiconductor Device Problems

38/2011 L. Neumann, A. Arnold, W. Hochhauser

Zur Stabilität von geklebten und geklotzten Glasscheiben: Beurteilung der Dunkerley'schen Geraden zur Beulwertbestimmung

37/2011 A. Dick, O. Koch, R. März, E. Weinmüller

Collocation Schemes for Nonlinear Index 1 DAEs with a Singular Point

Institute for Analysis and Scientific Computing

Vienna University of Technology

Wiedner Hauptstraße 8-10

1040 Wien, Austria

E-Mail: admin@asc.tuwien.ac.at

WWW: http://www.asc.tuwien.ac.at

FAX: $\quad+43-1-58801-10196$

ISBN 978-3-902627-05-6

(C) Alle Rechte vorbehalten. Nachdruck nur mit Genehmigung des Autors.

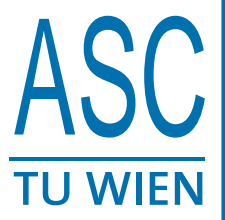




\title{
Indefinite Hamiltonian systems whose Titchmarsh-Weyl coefficients have no finite generalized poles of non-negative type
}

\author{
Matthias Langer, Harald WoraceK
}

\begin{abstract}
The two-dimensional Hamiltonian system

$(*) \quad y^{\prime}(x)=z J H(x) y(x), \quad x \in(a, b)$,

where the Hamiltonian $H$ takes non-negative $2 \times 2$-matrices as values, and $J:=\left(\begin{array}{cc}0 & -1 \\ 1 & 0\end{array}\right)$, has attracted a lot of interest over the past decades. Special emphasis has been put on operator models and direct and inverse spectral theorems. Weyl theory plays a prominent role in the spectral theory of the equation, relating the class of all equations $(*)$ to the class $\mathcal{N}_{0}$ of all Nevanlinna functions via the construction of Titchmarsh-Weyl coefficients.

In connection with the study of singular potentials, an indefinite (Pontryagin space) analogue of equation $(*)$ was proposed, where the 'general Hamiltonian' is allowed to have a finite number of inner singularities. Direct and inverse spectral theorems, relating the class of all general Hamiltonians to the class $\mathcal{N}_{<\infty}$ of all generalized Nevanlinna functions, were established.

In the present paper, we investigate the spectral theory of general Hamiltonians having a particular form, namely, such which have only one singularity and no non-trivial potential is present to the left of it. Our results can comprehensively be formulated as:

- We prove direct and inverse spectral theorems for this class, i.e. we establish an intrinsic characterization of the totality of all Titchmarsh-Weyl coefficients corresponding to general Hamiltonians of the considered form.

- We determine the asymptotic growth of the fundamental solution when approaching the singularity.

- We show that each solution of the equation has 'polynomially regularized' boundary values at the singularity.
\end{abstract}

Besides the intrinsic interest and depth of the presented results, our motivation is drawn from forthcoming applications: the present theorems form the core for our study of Sturm-Liouville equations with two singular endpoints and our further study of the structure theory of general Hamiltonians (both to be presented elsewhere).

AMS MSC 2010: Primary: 34L20, 34A55, 47B50. Secondary: 46E22, 37J99. Keywords: Hamiltonian system with inner singularity, Titchmarsh-Weyl coefficient, inverse problem, asymptotics of solutions

\section{Introduction}

In order to explain our present results and their significance, we need to recall the theory of positive definite and indefinite Hamiltonian systems up to 
a certain extent. We thus divide this introductory section into five parts: in the first two parts, we recall the required notions and facts, then we discuss in detail the present theorems, our motivation, and the organisation of the present manuscript.

Two-dimensional positive definite Hamiltonian systems.

Consider a Hamiltonian system of the form

$$
y^{\prime}(x)=z J H(x) y(x), \quad x \in(a, b),
$$

where $(a, b)$ is some (possibly unbounded) interval, $z$ is a complex parameter, $J:=\left(\begin{array}{cc}0 & -1 \\ 1 & 0\end{array}\right)$ and $H$ is a $2 \times 2$-matrix-valued locally integrable function defined on $(a, b)$ which takes real non-negative values and does not vanish on any set of positive measure. The function $H$ is called the Hamiltonian of the system (1.1).

With the system (1.1) a Hilbert space $L^{2}(H)$ and a (maximal) differential operator $T_{\max }(H)$ acting in this space is associated (actually, $T_{\max }(H)$ may be a linear relation, i.e. a multi-valued operator); see, e.g. $[\mathrm{K}],[\mathrm{HSW}]$ or §2.c below. The spectral properties of this operator highly depend on the growth of $H$ towards the endpoints $a$ and $b$. One says that Weyl's limit circle case prevails for $H$ at $a$ (or at $b$ ) if for one (and hence for all) $x_{0} \in(a, b)$,

$$
\int_{a}^{x_{0}} \operatorname{tr} H(x) d x<\infty \quad\left(\text { or } \int_{x_{0}}^{b} \operatorname{tr} H(x) d x<\infty\right),
$$

and one speaks of Weyl's limit point case at $a$ (or at $b$ ) if the respective integral diverges.

Assume that $H$ is in the limit circle case at $a$ and in the limit point case at $b$. Then a complex-valued function, its Titchmarsh-Weyl coefficient $q_{H}$, is associated with $H$ and constructed as follows. Let $\theta(x ; z)=\left(\theta_{1}(x ; z), \theta_{2}(x ; z)\right)^{T}$ and $\varphi(x ; z)=\left(\varphi_{1}(x ; z), \varphi_{2}(x ; z)\right)^{T}$ be the solutions of $(1.1)$ with initial values

$$
\theta(a ; z)=\left(\begin{array}{l}
1 \\
0
\end{array}\right), \quad \varphi(a ; z)=\left(\begin{array}{l}
0 \\
1
\end{array}\right)
$$

(it follows from the limit circle condition at $a$ that $H$ is integrable at $a$, and hence the initial value problem is well posed) so that the matrix function

$$
W_{H}(x ; z):=\left(\begin{array}{ll}
\theta_{1}(x ; z) & \theta_{2}(x ; z) \\
\varphi_{1}(x ; z) & \varphi_{2}(x ; z)
\end{array}\right)
$$

is (the transpose of) the fundamental solution of (1.1). The limit point condition at $b$ implies that, for each $z \in \mathbb{C} \backslash \mathbb{R}$, there exists a unique number $q_{H}(z) \in \mathbb{C}$ such that

$$
\theta(\cdot ; z)-q_{H}(z) \varphi(\cdot ; z) \in L^{2}(H)
$$

The function $q_{H}$ is called Titchmarsh-Weyl coefficient. Alternatively, it can be obtained as the limit (for $\tau \in \mathbb{R} \cup\{\infty\}$ )

$$
q_{H}(z)=\lim _{x \nearrow b} \frac{\theta_{1}(x ; z) \tau+\theta_{2}(x ; z)}{\varphi_{1}(x ; z) \tau+\varphi_{2}(x ; z)},
$$


which exists locally uniformly on $\mathbb{C} \backslash \mathbb{R}$ and does not depend on $\tau$. The Titchmarsh-Weyl coefficient $q_{H}$ belongs to the Nevanlinna class $\mathcal{N}_{0}$, i.e. it is analytic on $\mathbb{C} \backslash \mathbb{R}$, satisfies $q_{H}(\bar{z})=\overline{q_{H}(z)}, z \in \mathbb{C} \backslash \mathbb{R}$, and

$$
\operatorname{Im} z \cdot \operatorname{Im} q_{H}(z) \geq 0, \quad z \in \mathbb{C} \backslash \mathbb{R} .
$$

It plays a prominent role in the spectral theory of the system (1.1). For example, it generates, via its Herglotz integral representation, a measure $\mu_{H}$ and a Fourier transform from $L^{2}(H)$ onto $L^{2}\left(\mu_{H}\right)$. The Inverse Spectral Theorem by L. de Branges (see, e.g. [dB] and [Wi1]) states that the assignment

$$
H \longmapsto q_{H}
$$

sets up a bijective correspondence between the set of all Hamiltonians of the considered kind (up to reparameterization, i.e. changes of the independent variable) and the Nevanlinna class $\mathcal{N}_{0}$. In view of this fact it is an obvious task to try to translate properties of $H$ into properties of $q_{H}$. However, the mentioned Inverse Spectral Theorem is quite involved and (in general) non-constructive. Thus it is usually far from easy to find correspondences between properties of $H$ and $q_{H}$. For the purpose of illustration let us mention two theorems of this type. The Titchmarsh-Weyl coefficient $q_{H}$ belongs to the Stieltjes class (i.e. has an analytic continuation to $\mathbb{C} \backslash[0, \infty)$ and takes non-negative values on $(-\infty, 0))$ if and only if the Hamiltonian $H$ is of the form $\left(\right.$ where $\left.\xi_{\alpha}:=(\cos \alpha, \sin \alpha)^{T}\right)$

$$
H(x)=h(x) \cdot \xi_{-\phi(x)} \xi_{-\phi(x)}^{T}, \quad x \in(a, b) \text { a.e., }
$$

with real-valued functions $h(x), \phi(x)$ such that $h$ is non-negative integrable on $[a, b)$ and $\phi$ is non-decreasing with $\lim _{x \searrow a} \phi(x) \in[0, \pi)$ and $\lim _{x \nearrow b} \phi(x) \leq \pi$; see, [Wi2].

A second result of this type is the following: the Hamiltonian $H$ starts with an indivisible interval of type 0 , i.e.

$$
H(x)=h(x) \cdot \xi_{0} \xi_{0}^{T}=\left(\begin{array}{cc}
h(x) & 0 \\
0 & 0
\end{array}\right), \quad x \in(a, a+\varepsilon) \text { a.e. },
$$

for some $\varepsilon>0$ and some non-negative, locally integrable function $h$ on $(a, a+\varepsilon)$,

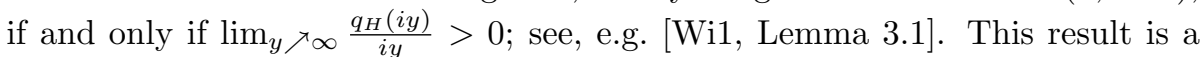
simple instance of a general intuitive idea, namely, that the behaviour of $q_{H}$ at infinity corresponds to the behaviour of $H$ at its left endpoint. Another, more involved, instance of the same principle can be found in [WW].

\section{The Pontryagin space analogue.}

In the theory of operators in spaces with an indefinite inner product an extension of the class $\mathcal{N}_{0}$ appeared and has proved to be useful: the so-called generalized Nevanlinna class $\mathcal{N}_{<\infty}$; see, e.g. [KL1], [KL2]. Thereby, instead of analytic functions, one considers meromorphic function on $\mathbb{C} \backslash \mathbb{R}$ and replaces condition (1.5) by requiring that the Nevanlinna kernel has a finite number of negative squares, cf. Definition 2.1 below. On the other side of the correspondence (1.6), i.e. within the framework of Hamiltonian systems, the extension of $\mathcal{N}_{0}$ to $\mathcal{N}_{<\infty}$ corresponds to admitting more general Hamiltonians $\mathfrak{h}$ which may have a finite number of inner singularities. Very roughly speaking, we may imagine a general Hamiltonian system of this kind as follows: 


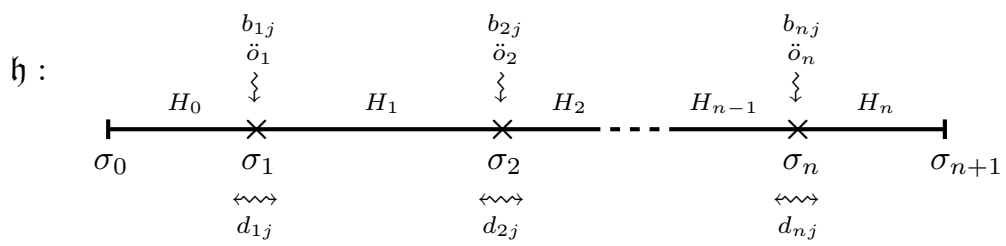

where $H_{i}$ are classical Hamiltonians which are not integrable on any side of the singularities $\sigma_{1}, \ldots, \sigma_{n}$, i.e. they are in Weyl's limit point case, where the data $\ddot{o}_{i}, b_{i j}$ describe what happens to a solution when passing through the singularity, and the data $d_{i j}$ describe a 'local interaction' of the potential to the left and to the right of the singularity. We say that the general Hamiltonian $\mathfrak{h}$ is regular if $H_{n}$ is in the limit circle case at $\sigma_{n+1}$. Otherwise, $\mathfrak{h}$ is called singular. The precise definitions are recalled in $\S 2$.d below.

For a general Hamiltonian $\mathfrak{h}$ an operator model consisting of a Pontryagin space boundary triple $(\mathcal{P}(\mathfrak{h}), T(\mathfrak{h}), \Gamma(\mathfrak{h}))$ was constructed, where $\mathcal{P}(\mathfrak{h})$ is a Pontryagin space, $T(\mathfrak{h})$ is the maximal operator (or linear relation) and $\Gamma(\mathfrak{h})$ are boundary mappings; see $[\mathrm{KW} / \mathrm{IV}]$. Analogues $\omega_{\mathfrak{h}}$ and $q_{\mathfrak{h}}$ of the fundamental solution $W_{H}$ and the Titchmarsh-Weyl coefficient $q_{H}$ were constructed, and a Fourier transform onto a space generated by a distribution $\phi_{\mathfrak{h}}$ instead of a measure $\mu_{H}$ was defined. An Inverse Spectral Theorem was proved which states that the assignment

$$
\mathfrak{h} \longmapsto q_{\mathfrak{h}}
$$

sets up a bijective correspondence between the set of all singular general Hamiltonians (up to reparameterization) and the generalized Nevanlinna class $\mathcal{N}_{<\infty}$; see $[\mathrm{KW} / \mathrm{V}],[\mathrm{KW} / \mathrm{VI}]$. The 'inverse' part of this theorem, i.e. that for each $q \in \mathcal{N}_{<\infty}$ there exists an essentially unique general Hamiltonian $\mathfrak{h}$ with $q=q_{\mathfrak{h}}$ is similarly involved and non-constructive as the corresponding result in the classical (positive definite) case. In the indefinite situation even the direct problem, i.e. the construction of the Titchmarsh-Weyl coefficient $q_{\mathfrak{h}}$ for given $\mathfrak{h}$, is nonconstructive.

\section{The present results.}

We investigate the following class(es) of general Hamiltonians:

1.1 Definition. Let $\alpha \in[0, \pi)$. We say that a general Hamiltonian $\mathfrak{h}$ belongs to the class $\mathfrak{H}_{\alpha}$, if

( $\left.\mathbf{g H}_{\mathbf{o . s .}}\right) \mathfrak{h}$ has exactly one singularity, i.e. is defined on a set of the form $\left(\sigma_{0}, \sigma_{1}\right) \cup\left(\sigma_{1}, \sigma_{2}\right)$;

$\left(\mathbf{g H}_{\alpha}\right) \quad H_{0}(x)=h_{0}(x) \cdot \xi_{\alpha} \xi_{\alpha}^{T}, x \in\left(\sigma_{0}, \sigma_{1}\right)$ a.e.

where again $\xi_{\alpha}=(\cos \alpha, \sin \alpha)^{T}$.

The results we are aiming for can be divided into three major themes.

Direct and inverse spectral theorems (Theorem 3.1). We show that a general Hamiltonian $\mathfrak{h}$ belongs to the class $\mathfrak{H}_{\alpha}$ if and only if its Titchmarsh-Weyl coefficient $q_{\mathfrak{h}}$ assumes the value $\cot \alpha$ at infinity with maximal possible multiplicity ${ }^{1}$.

\footnotetext{
${ }^{1}$ For the precise formulation of this terminology, see Definition 2.2 and Definition 2.3.
} 
For the proof of this result, we analyse the multi-valued part of a certain selfadjoint realization and use a classical result which connects the structure of the algebraic eigenspace at $\infty$ with the growth of the $Q$-function.

It is interesting to notice that the class of generalized Nevanlinna functions which appears in the present context as totally of Titchmarsh-Weyl coefficients, has already frequently appeared independently in earlier work ${ }^{2}$.

Asymptotic behaviour of the fundamental solution at a singularity (Theorem 4.1, Theorem 4.21). Let $\mathfrak{h} \in \mathfrak{H}_{\alpha}$ and consider the fundamental solution $\omega_{\mathfrak{h}}$ of the system. We show that three of the four entries of $\omega_{\mathfrak{h}}$ (if rotated appropriately according to the angle $\alpha$ ) pass continuously through the singularity, and determine precisely the rate of growth of the fourth entry. This is Theorem 4.1, the most involved and elaborate result of the paper. Its proof is based on an inductive procedure to reduce the negative index and on some classical results from complex analysis, in particular, the theory of de Branges spaces of entire functions.

Using the knowledge about $\mathfrak{H}_{\alpha}$ we can deduce a continuity result for the fundamental solution of an arbitrary general Hamiltonian $\mathfrak{h}$. Namely that, for each singularity of $\mathfrak{h}$, one row of $\omega_{\mathfrak{h}}$ (again, $\omega_{\mathfrak{h}}$ should be rotated appropriately) passes continuously through the singularity. Also, we determine the rate of growth of the other entries of $\omega_{\mathfrak{h}}$. This is Theorem 4.21; the proof uses some complex analysis and some standard methods from the theory of general Hamiltonians.

A noteworthy corollary for the classical 'positive definite' theory is that, for a certain kind of Hamiltonians $H$, the limit (1.4) defining the TitchmarshWeyl coefficient $q_{H}$ exists locally uniformly on the domain of analyticity of $q_{H}$ including intervals of the real line. The general formulation is Corollary 4.22.

Regularized boundary values (Theorem 5.1, Theorem 5.2). Let $\mathfrak{h} \in \mathfrak{H}_{\alpha}$ and let $\psi(\cdot ; z)$ be a solution of the corresponding differential equation (1.1) to the right of the singularity. We show that the projection of $\psi(\cdot ; z)$ onto a certain direction (depending on the value of $\alpha$ ) attains a boundary value at the singularity and that the projection onto the orthogonal direction can be regularized with ' $H$ polynomials' so that it attains a boundary value, cf. Theorem 5.1. We show that there exists a unique solution for which regularization is not necessary, cf. Theorem 5.2. The proof of these results relies heavily on the usage of a function space model for the boundary triple associated with $\mathfrak{h}$ (and, of course, the continuity result Theorem 4.1).

These results have two important corollaries which shed significant light on the behaviour of the system and at the influence of a singularity: first, Corollary 5.7 , which provides a fairly explicit way to compute the fundamental solution and the Titchmarsh-Weyl coefficient (i.e. to solve the direct spectral problem); second, Corollary 5.9, which shows explicitly how the data part of $\mathfrak{h}$ concentrated in the singularity and the respective local interaction parameters influence the Titchmarsh-Weyl coefficient.

\section{Motivation and forthcoming applications.}

A major motivation to study general Hamiltonians of the class $\mathfrak{H}_{\alpha}$ is that exactly this kind of general Hamiltonians appear when one investigates the spectral theory of classical (positive definite) Hamiltonian systems with two singular

\footnotetext{
${ }^{2}$ See, e.g. [DLS], [DHS], [KuLu] and the references therein. A posteriori, this is no surprise; in our forthcoming work [LW3], we will obtain a structural explanation.
} 
endpoints, for example systems that are connected with Sturm-Liouville equations with singular potentials like the Bessel equation. In fact, this was the origin of our studies (where we first realized the significance of the class $\mathfrak{H}_{\alpha}$ ). The Pontryagin space theory built up in this paper can be used to obtain knowledge about this positive definite situation. It is the basis for theorems asserting existence of singular boundary values, existence of Fourier transforms, inverse spectral theorems, etc. These topics will be presented in [LW3].

Besides this application to the theory of singular differential equations, the present results can be used to understand the structure of singularities and their influence on the solutions of the system in more detail. Intuitively speaking, the reason for this is that a singularity of an arbitrary general Hamiltonian behaves like the singularity of a general Hamiltonian of class $\mathfrak{H}_{\alpha}$ when approaching it from one side (either from the left or from the right). Local interaction between the two sides is more involved to capture, but can be handled by similar methods as used in the present manuscript. As a typical application of this principle, one can provide a method to solve the direct spectral problem for an arbitrary general Hamiltonian by means of integrating positive definite Hamiltonian systems; details are to be worked out and will be presented in a forthcoming publication.

Finally, it must be said that we find the presented theorems deep and interesting on their own right: partial continuity of the fundamental solution at a singularity is a striking and powerful property, the explained direct and inverse spectral theorem is a perfect instance of the mentioned intuitive principle that the behaviour of the Titchmarsh-Weyl coefficient at infinity is connected with the behaviour of the Hamiltonian at its left endpoint, the formula showing the influence of the parameters of the singularity on the Weyl coefficient is beautifully explicit and simple, etc.

\section{Organisation of the manuscript.}

In Section 2 we set up some notation and recall the definitions of the generalized Nevanlinna class, its subclasses under consideration and general Hamiltonians. Moreover, we provide some facts about the model associated with a general Hamiltonian and some useful tools. After this, the manuscript is naturally divided into section according to the above explained themes.

Table of contents

1. Introduction

2. Some preliminaries and supplements

3. Characterisation of Titchmarsh-Weyl coefficients

4. Partial continuity of the fundamental solution

\section{Some preliminaries and supplements}

Let us first fix some notation that is used throughout the paper. Set

$$
J:=\left(\begin{array}{cc}
0 & -1 \\
1 & 0
\end{array}\right), \quad \xi_{\phi}:=\left(\begin{array}{c}
\cos \phi \\
\sin \phi
\end{array}\right),
$$


and denote by $\mathbb{C}^{+}$the open upper half-plane, i.e. $\mathbb{C}^{+}:=\{z \in \mathbb{C}: \operatorname{Im} z>0\}$ and by $\mathbb{N}$ and $\mathbb{N}_{0}$ the set of positive and non-negative integers, respectively. For a $2 \times 2$-matrix $M=\left(m_{i j}\right)_{i, j=1}^{2}$ and a scalar $\tau \in \mathbb{C}$ we set

$$
M \star \tau:= \begin{cases}\frac{m_{11} \tau+m_{12}}{m_{21} \tau+m_{22}}, & \tau \in \mathbb{C}, \\ \frac{m_{11}}{m_{21}}, & \tau=\infty .\end{cases}
$$

It is easy to see that $M \star(N \star \tau)=(M N) \star \tau$ if $N$ is another $2 \times 2$-matrix.

The rest of this section is divided into subsections as follows. In $\S 2$.a and $\S 2$.b we recall definitions and properties of certain classes of holomorphic functions. In §2.c the notion of boundary triples is recalled in a form that is used in the paper. Properties of classical (positive definite) Hamiltonian systems are reviewed in $\S 2$.d, whereas in $\S 2$.e the definition of general Hamiltonians is given. In $\S 2$.f a certain class of general Hamiltonians with one singularity is studied and a function space operator model is described in detail. Finally, in $§ 2$.g rotation isomorphism are recalled, a technical tool which is used in later proofs.

\section{a. The generalized Nevanlinna class.}

We recall the definition of the class $\mathcal{N}_{<\infty}$ of generalized Nevanlinna functions.

2.1 Definition. Let $q$ be a complex-valued function and let $\kappa \in \mathbb{N}_{0}$. We write $q \in \mathcal{N}_{\kappa}$ if

(N1) $q$ is real (meaning $q(\bar{z})=\overline{q(z)}$ ) and meromorphic on $\mathbb{C} \backslash \mathbb{R}$;

(N2) with $D$ denoting the domain of holomorphy of $q$, the Nevanlinna kernel (for $z=\bar{w}$ this formula should be interpreted appropriately as a derivative)

$$
N_{q}(w, z):=\frac{q(z)-\overline{q(w)}}{z-\bar{w}}, \quad z, w \in D
$$

has $\kappa$ negative squares on $D$. The latter means that for every choice of $n \in \mathbb{N}$ and $z_{1}, \ldots, z_{n} \in D$ the matrices $\left(N_{q}\left(z_{i}, z_{j}\right)\right)_{i, j=1}^{n}$ have at most $\kappa$ negative eigenvalues and for at least one choice of $n$ and $z_{i}$ the matrix has exactly $\kappa$ negative eigenvalues.

We agree that the constant function with value $\infty$ belongs to $\mathcal{N}_{0}$. Further, we set

$$
\mathcal{N}_{<\infty}:=\bigcup_{\kappa \in \mathbb{N}_{0}} \mathcal{N}_{\kappa}
$$

and write ind $-q=\kappa$ to express that $q \in \mathcal{N}_{<\infty}$ belongs to $\mathcal{N}_{\kappa}$. The set $\mathcal{N}_{<\infty}$ is called the class of generalized Nevanlinna functions.

It is a classical result that the class $\mathcal{N}_{0} \backslash\{\infty\}$ consists of those functions $q$ that are holomorphic on $\mathbb{C} \backslash \mathbb{R}$, are real and satisfy $\operatorname{Im} z \cdot \operatorname{Im} q(z) \geq 0$ for $z \in \mathbb{C} \backslash \mathbb{R}$. This fact goes back to as far as $[\mathrm{H}]$ or $[\mathrm{P}]$.

A generalized Nevanlinna function cannot grow arbitrarily fast towards $\infty$. In fact, for each $q \in \mathcal{N}_{\kappa}$, the limit

$$
\lim _{z \rightarrow i \infty} \frac{q(z)}{z^{2 \kappa+1}} \quad \text { exists and is in }[0, \infty) \text {. }
$$


Here $\hat{\rightarrow}$ denotes a non-tangential limit, i.e. a limit inside a sector of the form $\{z \in \mathbb{C}: \arg \in[\alpha, \pi-\alpha]\}$ with $\alpha \in\left(0, \frac{\pi}{2}\right)$. This fact has been shown, e.g. in [L]. Even more precise knowledge about the power growth of generalized functions is available. Using the canonical factorization of a generalized Nevanlinna function established in [DLLS], one can easily deduce that for each $q \in \mathcal{N}_{\kappa}$ there exists a unique non-negative integer $n$, not exceeding $\kappa$, such that

$$
\lim _{z \rightarrow i \infty} \frac{q(z)}{z^{2 n+1}} \in[0, \infty) \quad \text { but } \quad \lim _{z \rightarrow i \infty} \frac{q(z)}{z^{2 n-1}} \in(-\infty, 0) \vee \lim _{z \rightarrow i \infty}\left|\frac{q(z)}{z^{2 n-1}}\right|=\infty
$$

In the present paper the subclass of $\mathcal{N}_{<\infty}$ appears, which consists of all functions having, in this sense, maximal possible growth at infinity.

2.2 Definition. For $\kappa \in \mathbb{N}$ we denote by $\mathcal{N}_{\kappa}^{(\infty)}$ the set of all functions $q \in \mathcal{N}_{\kappa}$ such that

$$
\lim _{z \rightarrow i \infty} \frac{q(z)}{z^{2 \kappa-1}} \in(-\infty, 0) \quad \text { or } \quad \lim _{z \rightarrow i \infty}\left|\frac{q(z)}{z^{2 \kappa-1}}\right|=\infty,
$$

where $\hat{\rightarrow}$ again denotes the non-tangential limit. Moreover, we set

$$
\mathcal{N}_{<\infty}^{(\infty)}:=\bigcup_{\kappa \in \mathbb{N}} \mathcal{N}_{\kappa}^{(\infty)}
$$

The class $\mathcal{N}_{<\infty}^{(\infty)}$ previously appeared in many papers in the context of SturmLiouville equations with singular endpoints or singular perturbations, see [DLS], [DHS], [DKuS], [DLSZ], [DLuS1], [DLuS2], [DLuS3], [KuLu].

Slightly more general, we also consider the subclasses of $\mathcal{N}_{<\infty}$ of all functions which attain a certain value $\tau \in \mathbb{R}$ at $\infty$ with maximal possible multiplicity.

2.3 Definition. Let $\tau \in \mathbb{R}$. We denote by $\mathcal{N}_{\kappa}^{(\tau)}$ the set of all functions $q \in \mathcal{N}_{\kappa}$ such that

$$
\frac{1}{\tau-q(z)} \in \mathcal{N}_{\kappa}^{(\infty)} \text {. }
$$

Further, we set

$$
\mathcal{N}_{<\infty}^{(\tau)}:=\bigcup_{\kappa \in \mathbb{N}} \mathcal{N}_{\kappa}^{(\tau)}
$$

2.4 Remark. Let $\kappa \in \mathbb{N}$ and $\tau \in \mathbb{R}$. Then a function $q \in \mathcal{N}_{\kappa}$ belongs to $\mathcal{N}_{\kappa}^{(\tau)}$ if and only if

$$
\lim _{z \rightarrow i \infty} z^{2 \kappa-1}(q(z)-\tau) \in[0, \infty) .
$$

The class $\mathcal{N}_{<\infty}^{(\infty)}$ admits an operator theoretic interpretation. In the language of [KL3] and [L], the condition (2.3) means that $\infty$ is a generalized pole of nonpositive type with degree of non-positivity equal to ind $q$. Equivalently, one can say that $q$ has a generalized pole of non-positive type at $\infty$ with maximal possible degree of non-positivity permitted by the negative index and, consequently, no finite generalized poles of non-positive type. More precisely, the statement in 
the following lemma is true, which follows, e.g. from [L, Theorem 3.2] and which is used in Section 3. Recall that the algebraic eigenspace at infinity of a linear relation $A$ in a Pontryagin space $\mathcal{P}$ is the set of all elements of Jordan chains at $\infty$, where a Jordan chain at $\infty$ is a sequence of vectors $g_{0}, \ldots, g_{n} \in \mathcal{P}$ with $g_{0}=0$ such that $\left(g_{i-1} ; g_{i}\right) \in A$ for all $i=1, \ldots, n$.

2.5 Lemma. Let $q \in \mathcal{N}_{\kappa}$. Moreover, let $A$ be a self-adjoint relation in a Pontryagin space $(\mathcal{P},[\cdot, \cdot])$ and $v \in \mathcal{P}$ such that

$$
q(z)=\overline{q\left(z_{0}\right)}+\left(z-z_{0}\right)\left[\left(I+\left(z-z_{0}\right)(A-z)^{-1}\right) v, v\right], \quad z \in \rho(A),
$$

where $z_{0} \in \rho(A)$ is fixed and assume that this representation is minimal, i.e.

$$
\mathcal{P}=\text { c.l.s. }\left\{\left(I+\left(z-z_{0}\right)(A-z)^{-1}\right) v: z \in \rho(A)\right\}
$$

where c.l.s. stands for 'closed linear span'. Then

$$
q \in \mathcal{N}_{\kappa}^{(\infty)} \Longleftrightarrow \nu_{\infty}(A)=\kappa
$$

where $\nu_{\infty}(A)$ is the degree of non-positivity of $\infty$, i.e. the maximal dimension of a non-positive $A^{-1}$-invariant subspace of the algebraic eigenspace at infinity of $A$.

\section{b. Some classes of entire functions.}

In this subsection we recall several classes of scalar and matrix-valued entire functions, which are needed in the proofs in later sections. Note that an entire function is called real if $f(\bar{z})=\overline{f(z)}$ for all $z \in \mathbb{C}$. Moreover, we set $f^{\#}(z):=$ $\overline{f(\bar{z})}$. First we recall the definition of the Pólya class; for details see, e.g. [dB, Section 7].

2.6 Definition. An entire function $f$ belongs to the Pólya class if

(P1) $f$ has no zeros in the upper half-plane $\mathbb{C}^{+}$;

(P2) $f$ satisfies $|f(z)| \geq|f(\bar{z})|$ for $z \in \mathbb{C}^{+}$;

(P3) for each fixed $x \in \mathbb{R}$ the function $y \mapsto|f(x+i y)|$ is non-decreasing on $(0, \infty)$.

Let us next consider functions of bounded type; see, e.g. [RR94, Definition 3.15 and Theorem 3.20] or [dB, Section 8].

2.7 Definition. A function that is analytic in the upper half-plane $\mathbb{C}^{+}$is said to be of bounded type if it can be written as a quotient $f(z)=p(z) / q(z)$ of two analytic functions which are bounded throughout $\mathbb{C}^{+}$and where $q$ is not identically equal to 0 .

According to $[\mathrm{dB}$, Problem 24] it is possible to choose $p$ and $q$ such that $q$ has no zeros in $\mathbb{C}^{+}$. One can define bounded type in the lower half-plane in a similar way.

2.8 Remark. 
(i) A function from $\mathcal{N}_{<\infty}$ is of bounded type in the upper and lower halfplanes; see, e.g. [KW/I, Proposition 2.4].

(ii) According to $[\mathrm{dB}$, Problem 34] an entire function $f$ that satisfies $|f(z)| \geq$ $|f(\bar{z})|$ for $z \in \mathbb{C}^{+}$and is of bounded type in the upper half-plane belongs to the Pólya class. In particular, a real entire function that is of bounded type in the upper half-plane belongs to the Pólya class.

Next we recall a generalization of Hermite-Biehler functions, namely functions belonging to the class $\mathcal{H} B_{\kappa}$. When $E: D \rightarrow \mathbb{C}$ is an analytic function defined on some open subset $D$ of the complex plane, we define a kernel $K_{E}$ as

$$
K_{E}(w, z):=\frac{i}{2} \cdot \frac{E(z) \overline{E(w)}-E^{\#}(z) \overline{E^{\#}(w)}}{z-\bar{w}}, \quad z, w \in D .
$$

For $z=\bar{w}$ this formula has to be interpreted appropriately as a derivative, which is possible by analyticity. For more details see, e.g. [KW/V, §2.e].

2.9 Definition. Let $E$ be a complex-valued function defined on $\mathbb{C}$ and let $\kappa \in \mathbb{N}_{0}$. We write $E \in \mathcal{H} B_{\kappa}$ if

(HB1) $E$ is entire;

(HB2) $E$ and $E^{\#}$ have no common non-real zeros;

(HB3) the kernel $K_{E}$ has $\kappa$ negative squares on $\mathbb{C}$.

We use the notation

$$
\mathcal{H} B_{<\infty}:=\bigcup_{\kappa \in \mathbb{N}_{0}} \mathcal{H} B_{\kappa}
$$

and write ind $E=\kappa$ to express that a function $E \in \mathcal{H} B_{<\infty}$ belongs to $\mathcal{H} B_{\kappa}$. The class $\mathcal{H} B_{<\infty}$ is called the indefinite Hermite-Biehler class.

It is a classical result that an entire function $E$ belongs to the class $\mathcal{H} B_{0}$ if and only if either it is a constant multiple of a real entire function which has no non-real zeros, or it satisfies $|E(z)|>|E(\bar{z})|$ for $z \in \mathbb{C}^{+}$. For details see, e.g. [Le, Chapter 7].

By means of the reproducing kernel $K_{E}$, each function $E \in \mathcal{H} B_{<\infty}$ generates a Pontryagin space $\mathfrak{P}(E)$ which consists of entire functions. This space is referred to as the de Branges Pontryagin space generated by $E$; see [ADSR] and $[\mathrm{KW} / \mathrm{I}]$.

The indefinite Hermite-Biehler class is related to the generalized Nevanlinna class: let $E(z) \in \mathcal{H} B_{<\infty}$ and write $E=A-i B$ with the real entire functions

$$
A:=\frac{1}{2}\left(E+E^{\#}\right), \quad B:=\frac{i}{2}\left(E-E^{\#}\right) .
$$

Then $q=\frac{B}{A}$ belongs to $\mathcal{N}_{<\infty}$ with ind $q=$ ind $_{-} E$. This follows from the relation

$$
K_{E}(w, z)=A(z) N_{q}(w, z) \overline{A(w)}, \quad z, w \in \mathbb{C}, A(z), A(w) \neq 0 .
$$


Finally in this subsection, let us define a class of matrix-valued entire functions. Functions from this class appear later as fundamental solutions of general Hamiltonians. When $W$ is an entire $2 \times 2$-matrix-valued function which satisfies $W(z) J W(\bar{z})^{*}=J$ for $z \in \mathbb{C}$, then a kernel $H_{W}$ is defined by

$$
H_{W}(w, z):=\frac{W(z) J W(w)^{*}-J}{z-\bar{w}}, \quad z, w \in \mathbb{C} .
$$

For $z=\bar{w}$ this formula has to be interpreted appropriately as a derivative.

2.10 Definition. Let $W=\left(w_{i j}\right)_{i, j=1}^{2}$ be a $2 \times 2$-matrix-valued function and let $\kappa \in \mathbb{N}_{0}$. We write $W \in \mathcal{M}_{\kappa}$ if

(M1) the entries $w_{i j}$ of $W$ are real entire functions;

(M2) $\operatorname{det} W(z)=1$ for $z \in \mathbb{C}$, and $W(0)=I$;

(M3) the kernel $H_{W}$ has $\kappa$ negative squares on $\mathbb{C}$.

Note that the conditions (M1) and (M2) together imply that $W(z) J W(\bar{z})^{*}=J$. Moreover, we set

$$
\mathcal{M}_{<\infty}:=\bigcup_{\nu \in \mathbb{N}_{0}} \mathcal{M}_{\nu}
$$

and write ind $-W=\kappa$ to express that $W \in \mathcal{M}_{\kappa}$.

Define a map $t: \mathcal{M}_{<\infty} \rightarrow \mathbb{R}$ by

$$
\mathfrak{t}(W):=\operatorname{tr}\left(W^{\prime}(0) J\right)=w_{12}^{\prime}(0)-w_{21}^{\prime}(0)
$$

for $W=\left(w_{i j}\right)_{i, j=1}^{2} \in \mathcal{M}_{<\infty}$. This map $\mathfrak{t}$ is used, e.g. to measure the growth of the unbounded entry of the fundamental solution of an indefinite Hamiltonian.

Each matrix $W \in \mathcal{M}_{<\infty}$ generates, by means of the kernel $H_{W}$, a reproducing kernel Pontryagin space $\mathfrak{K}(W)$ whose elements are 2-vector-valued entire functions; see, e.g. [ADSR] and [KW/V, §2.a].

If $W=\left(w_{i j}\right)_{i, j=1}^{2} \in \mathcal{M}_{<\infty}$, then the function $E(z)=w_{11}(z)-i w_{12}(z)$ belongs to $\mathcal{H} B_{<\infty}$ with ind $-E \leq$ ind $-W$, which follows from the relation

$$
K_{E}(w, z)=(1,0) H_{W}(w, z)\left(\begin{array}{l}
1 \\
0
\end{array}\right) \quad z, w \in \mathbb{C} ;
$$

cf. $[\mathrm{KW} / \mathrm{V}, \S 2 . \mathrm{e}]$. Hence $\frac{w_{12}}{w_{11}}$ belongs to $\mathcal{N}_{<\infty}$.

\section{c. Boundary triples.}

Let us also recall the notion of boundary triples as introduced in $[\mathrm{KW} / \mathrm{IV}$, Definition 2.7].

2.11 Definition. A triple $(\mathcal{P}, T, \Gamma)$ is called a boundary triple if

(i) $(\mathcal{P},[\cdot, \cdot])$ is a Pontryagin space, which carries a conjugate linear and antiisometric involution $-: \mathcal{P} \rightarrow \mathcal{P}$;

(ii) $T$ is a closed linear relation in $\mathcal{P}$ that is real, i.e.

$$
(f ; g) \in T \Longleftrightarrow(\bar{f} ; \bar{g}) \in T
$$


(iii) $\Gamma \subseteq T \times\left(\mathbb{C}^{2} \times \mathbb{C}^{2}\right)$ is a closed linear relation with dom $\Gamma=T$, which is compatible with the involution $-: \mathcal{P} \rightarrow \mathcal{P}$ in the sense that

$$
((f ; g) ;(a ; b)) \in \Gamma \Longleftrightarrow((\bar{f} ; \bar{g}) ;(\bar{a} ; \bar{b})) \in \Gamma ;
$$

(iv) the following abstract Green identity holds:

$$
\begin{array}{r}
{[g, u]-[f, v]=\left(\begin{array}{l}
c \\
d
\end{array}\right)^{*}\left(\begin{array}{cc}
J & 0 \\
0 & -J
\end{array}\right)\left(\begin{array}{l}
a \\
b
\end{array}\right)} \\
\text { when }((f ; g) ;(a ; b)),((u ; v) ;(c ; d)) \in \Gamma ;
\end{array}
$$

(v) $\operatorname{ker} \Gamma=T^{*}$.

In applications, e.g. to differential operators, often the relation $T$ in a boundary triple $(\mathcal{P}, T, \Gamma)$ is the maximal relation, e.g. the differential operator with no boundary conditions imposed; it is the adjoint of a symmetric relation. The relation $\Gamma$ often maps the functions in the domain of $T$ (or more precisely, pairs in the relation $T$ ) onto the boundary values at the left and/or right endpoint of the interval or linear combinations of them. The abstract Green identity is then nothing else than a classical Green or Lagrange identity, which follows from integration by parts. Note that $a, b, c, d$ in the abstract Green identity are 2 -vectors.

Two boundary triples $(\mathcal{P}, T, \Gamma),(\tilde{\mathcal{P}}, \tilde{T}, \tilde{\Gamma})$ are called isomorphic if there exists a pair $(\varpi, \phi)$ (which is then called an isomorphism) such that

(i) $\varpi$ is an isometric isomorphism from $\mathcal{P}$ onto $\tilde{\mathcal{P}}$ that is compatible with the respective involutions in the sense that $\varpi(\bar{x})=\overline{\varpi(x)}$ for $x \in \mathcal{P}$;

(ii) $\phi$ is an isometric isomorphism from $\left(\mathbb{C}^{2} \times \mathbb{C}^{2},\left(\left(\begin{array}{cc}J & 0 \\ 0 & -J\end{array}\right) \cdot, \cdot\right)\right)$ onto itself;

(iii) $(\varpi \times \varpi)(T)=\tilde{T}$;

(iv) $\left.\tilde{\Gamma} \circ(\varpi \times \varpi)\right|_{T}=\phi \circ \Gamma$.

2.12 Remark. For certain boundary triples one can construct a matrix function $\omega$ from the class $\mathcal{M}_{\kappa}$ and an isometric isomorphism $\Xi$ from $\mathcal{P}$ onto the reproducing kernel space $\mathfrak{K}(\omega)$ such that the symmetric operator $T^{*}$ corresponds to the operator of multiplication by the independent variable in the space $\mathfrak{K}(\omega)$; for details see $[\mathrm{KW} / \mathrm{V}$, Definition 4.3 and Theorem 4.19]. This construction is related to Krein's representation of entire operators.

\section{d. More facts about Hamiltonian systems.}

A function $H:(a, b) \rightarrow \mathbb{R}^{2 \times 2}$, where $(a, b)$ is an interval with $-\infty \leq a<b \leq \infty$, is called a Hamiltonian if

the values of $H$ are real non-negative matrices and the function $H$ is

locally integrable and does not vanish on any set of positive measure.

Recall the definition of Weyl's limit point and limit circle cases from the introduction; see (1.2). It follows easily from the non-negativity of $H$ that limit circle case prevails at $a$ if and only if $H$ is integrable at $a$, i.e. $a$ is a regular endpoint. 
In connection with the differential equation (1.1) one often considers also the matrix differential equation

$$
\frac{\partial}{\partial x} W(x ; z) J=z W(x ; z) H(x), \quad x \in(a, b),
$$

where $W(x ; z)$ is a $2 \times 2$-matrix for $x \in(a, b), z \in \mathbb{C}$. If $a \in \mathbb{R}$ and (1.1) is in the limit circle case at $a$ (i.e. it is regular at $a$ ), then the differential equation (2.7) together with the initial condition $W(a ; z)=I$ has a unique solution, which we denote by $W_{H}$, cf. (1.3). The rows of $W_{H}$ satisfy the differential equation (1.1) and $y(x)=\left[\left(a_{1}, a_{2}\right) W_{H}(x ; z)\right]^{T}$ is a solution of (1.1) satisfying the initial conditions $y(a)=\left(a_{1}, a_{2}\right)^{T}$. Moreover, if $W_{H, x_{1}}(x ; z)$ denotes the solution of (2.7) satisfying the initial condition $W_{H, x_{1}}\left(x_{1} ; z\right)=I$ with some $x_{1} \in(a, b)$, then

$$
W_{H}(x ; z)=W_{H}\left(x_{1} ; z\right) W_{H, x_{1}}(x ; z)
$$

for $x \in\left[x_{1}, b\right)$.

2.13. Properties of $W_{H}$. Assume that (1.1) is in the limit circle case at $a$. For fixed $x \in(a, b)$ the matrix function $W_{H}(x ; \cdot)$ belongs to the class $\mathcal{M}_{0}$, where $\mathcal{M}_{0}$ was defined in Definition 2.10. If one combines Theorems 38, 27 and 25 in [dB], then it follows that the entries $W_{H}(x ; \cdot)_{i j}, i, j=1,2$, of $W_{H}(x ; \cdot)$ are of bounded type in the upper half-plane, and since the functions are real, also in the lower half-plane. By Remark $2.8($ ii $)$ this implies that $W_{H}(x ; \cdot)_{i j}$ belongs to the Pólya class. Since $W_{H}(x ; 0)_{12}=W_{H}(x ; 0)_{21}=0$, it follows from [dB, $\S 1.7$, Lemma 1] that

$$
\frac{W_{H}(x ; z)_{12}}{z} \text { and } \frac{W_{H}(x ; z)_{21}}{z}
$$

are also from the Pólya class. Moreover, the function $E(z)=W_{H}(x ; z)_{11}-$ $i W_{H}(x ; z)_{12}$ belongs to the Hermite-Biehler class $\mathcal{H} B_{0}$. Hence $E$ induces a de Branges Hilbert space $\mathfrak{P}(E)$ of entire functions and $\frac{W_{H}(x ; \cdot)_{12}}{W_{H}(x ; \cdot)_{11}} \in \mathcal{N}_{0}$; see $\S 2 . \mathrm{b}$ and $[\mathrm{dB}]$.

With the notation (2.2) the Titchmarsh-Weyl coefficient $q_{H}$ for (1.1) (as defined in (1.4)) can be written as

$$
q_{H}(z)=\lim _{x \nearrow b} W_{H}(x ; z) \star \tau, \quad z \in \mathbb{C} \backslash \mathbb{R},
$$

for $\tau \in \mathbb{R} \cup\{\infty\}$, where the limit exists locally uniformly in $\mathbb{C} \backslash \mathbb{R}$ and is independent of $\tau$.

With $\mathfrak{t}$ from (2.5) we have

$$
\mathfrak{t}\left(W_{H}\left(x_{2} ; \cdot\right)\right)-\mathfrak{t}\left(W_{H}\left(x_{1} ; \cdot\right)\right)=\int_{x_{1}}^{x_{2}} \operatorname{tr} H(x) d x
$$

for $a \leq x_{1}<x_{2}<b$ as can easily be seen.

2.14. Indivisible intervals. An interval $(\alpha, \beta) \subseteq(a, b)$ is called $H$-indivisible of type $\phi$ if

$$
H(x)=h(x) \xi_{\phi} \xi_{\phi}^{T}, \quad x \in(\alpha, \beta),
$$

where $\xi_{\phi}$ is defined in (2.1) and $h$ is a locally integrable function that is positive almost everywhere. If $(\alpha, \beta)$ is $H$-indivisible, then, for $\alpha<x_{1}<x_{2}<\beta$,

$$
W_{H}\left(x_{1} ; z\right)^{-1} W_{H}\left(x_{2} ; z\right)=W_{(l, \phi)}(z)
$$


where

$$
W_{(l, \phi)}(z):=\left(\begin{array}{cc}
1-l z \sin \phi \cos \phi & l z \cos ^{2} \phi \\
-l z \sin ^{2} \phi & 1+l z \sin \phi \cos \phi
\end{array}\right)
$$

and

$$
l=\int_{x_{1}}^{x_{2}} h(x) d x .
$$

The number $\int_{\alpha}^{\beta} h(x) d x$ is called the length of the indivisible interval $(\alpha, \beta)$, which is infinite exactly when $H$ is singular at $\alpha$ or $\beta$.

2.15. The maximal relation $T_{\max }(H)$. With a Hamiltonian $H$ a maximal linear relation (i.e. a multi-valued operator), $T_{\max }$, can be associated as follows (for details see, e.g. [KW/IV, §2]). First we recall the definition of the space $L^{2}(H)$ : it is the space of measurable functions $f$ defined on $(a, b)$ with values in $\mathbb{C}^{2}$ which satisfy $\int_{a}^{b} f^{*} H f<\infty$ and have the property that $\xi_{\phi}^{T} f$ is constant on every indivisible interval of type $\phi$, factorized with respect to the equivalence relation $=_{H}$ where

$$
f=_{H} g \quad \Longleftrightarrow \quad H(f-g)=0 \quad \text { a.e. }
$$

In the space $L^{2}(H)$ the maximal relation $T_{\max }(H)$ is defined as

$$
\begin{aligned}
T_{\max }(H):=\{( & (f ; g) \in\left(L^{2}(H)\right)^{2}: \exists \text { representatives } \hat{f}, \hat{g} \text { of } f, g \text { such that } \\
& \left.\hat{f} \text { is locally absolutely continuous and } \hat{f}^{\prime}=J H \hat{g} \text { a.e. on }(a, b)\right\} .
\end{aligned}
$$

Sometimes we need Green's identity in the following form: if $f$ and $u$ are absolutely continuous functions on $\left[x_{1}, x_{2}\right]$ where $a<x_{1}<x_{2}<b$ and $g, v$ are such that

$$
f^{\prime}=J H g, \quad u^{\prime}=J H v, \quad \text { a.e. on }\left(x_{1}, x_{2}\right),
$$

then

$$
\int_{x_{1}}^{x_{2}} u^{*} H g-\int_{x_{1}}^{x_{2}} v^{*} H f=u\left(x_{1}\right)^{*} J f\left(x_{1}\right)-u\left(x_{2}\right)^{*} J f\left(x_{2}\right) ;
$$

see $[\mathrm{KW} / \mathrm{IV}$, Remark 2.20].

With a Hamiltonian $H$ defined on an interval $(a, b)$ also a boundary relation $\Gamma(H)$ is associated: a pair $((f ; g) ;(c ; d))$ belongs to $\Gamma(H)$ if and only if there exists a representative $\hat{f}$ of $f$ that is absolutely continuous such that

$$
c=\left\{\begin{array}{ll}
\hat{f}(a) & \text { if } H \text { is regular at } a, \\
0 & \text { if } H \text { is singular at } a,
\end{array} \quad d= \begin{cases}\hat{f}(b) & \text { if } H \text { is regular at } b, \\
0 & \text { if } H \text { is singular at } b .\end{cases}\right.
$$

For details see, e.g. [KW/IV, Theorems 2.18 and 2.19].

Let us also recall some properties of Hamiltonian systems which are connected with the behaviour of $H$ at an endpoint. They are needed in the definition of general (indefinite) Hamiltonians in $§ 2 . e$. 
2.16. Hilbert-Schmidt resolvents. Let $H$ be a Hamiltonian defined on some interval $(a, b)$.

(i) We say that $H$ satisfies the condition $\left(\mathrm{HS}_{-}\right)$if for one (and hence for all) $x_{0} \in(a, b)$ the resolvents of self-adjoint extensions of $\left(T_{\max }\left(\left.H\right|_{\left(a, x_{0}\right)}\right)\right)^{*}$, i.e. self-adjoint restrictions of $T_{\max }\left(\left.H\right|_{\left(a, x_{0}\right)}\right)$, are Hilbert-Schmidt operators. Similarly, we say that $\left(\mathrm{HS}_{+}\right)$holds for $H$ if for one (and hence for all) $x_{0} \in(a, b)$ the resolvents of self-adjoint extensions of $\left(T_{\max }\left(\left.H\right|_{\left(x_{0}, b\right)}\right)\right)^{*}$ are Hilbert-Schmidt operators, cf. [KW/IV, §2.3.a].

(ii) It follows, with an obvious change of variable, from $[\mathrm{KW} / \mathrm{IV}$, Theorem 2.27 that these properties can be characterized explicitly in terms of $H$. Namely, $H$ satisfies (HS_) if and only if there exists a number $\phi \in \mathbb{R}$ such that for one (and hence for all) $x_{0} \in(a, b)$

$\left(\mathbf{I}_{\phi}^{-}\right)$

$$
\int_{a}^{x_{0}} \xi_{\phi}^{T} H(x) \xi_{\phi} d x<\infty,
$$

where $\xi_{\phi}$ is defined in $(2.1)$;

$\left(\mathbf{H S}_{\phi}^{-}\right)$with $M(x):=\int_{x_{0}}^{x} H(y) d y$,

$$
\left|\int_{a}^{x_{0}} \xi_{\phi+\frac{\pi}{2}}^{T} M(x) \xi_{\phi+\frac{\pi}{2}} \xi_{\phi}^{T} H(x) \xi_{\phi} d x\right|<\infty .
$$

If $H$ is in the limit point case at $a$ and satisfies (HS-), then the number $\phi \in[0, \pi)$ such that $\left(\mathrm{I}_{\phi}^{-}\right)$and $\left(\mathrm{HS}_{\phi}^{-}\right)$hold is uniquely determined; in this case we denote this unique $\phi$ by $\phi_{-}(H)$. Clearly, $\phi_{-}(H)$ does not depend on the choice of the cutting point $x_{0}$.

The property $\left(\mathrm{HS}_{+}\right)$is characterized by corresponding conditions $\left(\mathrm{I}_{\phi}^{+}\right)$, $\left(\mathrm{HS}_{\phi}^{+}\right)$. The unique angle $\phi$ is denoted by $\phi_{+}(H)$ in this case, and again does not depend on $x_{0}$.

Let us explicitly consider the case $\phi=0$; the conditions $\left(\mathrm{I}_{0}^{-}\right)$and $\left(\mathrm{HS}_{0}^{-}\right)$ read as follows:

$$
\begin{aligned}
\left(\mathrm{I}_{0}^{-}\right): & \int_{a}^{x_{0}} h_{11}(x) d x<\infty, \\
\left(\mathrm{HS}_{0}^{-}\right): & \int_{a}^{x_{0}} \int_{x}^{x_{0}} h_{22}(t) d t h_{11}(x) d x<\infty .
\end{aligned}
$$

For $\left(\mathrm{I}_{\frac{\pi}{2}}^{-}\right),\left(\operatorname{HS}_{\frac{\pi}{2}}^{-}\right)$one has to swap $h_{11}$ and $h_{22}$.

If $(a, c)$, with some $c \in(a, b)$, is an indivisible interval of type $\phi$ and $H$ is in the limit point case at $a$, then $\left(\mathrm{I}_{\phi-\frac{\pi}{2}}^{-}\right)$and $\left(\mathrm{HS}_{\phi-\frac{\pi}{2}}^{-}\right)$are satisfied but $\left(\mathrm{I}_{\phi^{\prime}}^{-}\right),\left(\mathrm{HS}_{\phi^{\prime}}^{-}\right)$are not when $\phi-\frac{\pi}{2}-\phi^{\prime} \notin \pi \mathbb{Z}$. 
2.17. Some growth condition. Let $H$ be a Hamiltonian defined on some interval $(a, b)$ which satisfies $\left(\mathrm{HS}_{-}\right)$and fix a point $x_{0} \in(a, b)$. Denote by $\mathcal{I}$ the operator $f \mapsto \int_{x_{0}}^{x} J H(t) f(t) d t$. Then there exist numbers $\rho_{k} \in \mathbb{R}, k \in \mathbb{N}_{0}$, such that $\rho_{0} \neq 0$ and

$$
\sum_{k=0}^{n} \rho_{n-k} \mathcal{I}^{k} \xi_{\phi_{-}(H)} \in L^{2}\left(\left.H\right|_{\left(a, x_{0}\right)}\right), \quad n \in \mathbb{N}_{0}
$$

see $\left[\mathrm{KW} / \mathrm{IV}\right.$, Corollary 3.5]. If $\xi_{\phi_{-}(H)}$ is replaced by $\xi_{\phi_{-}(H)+\frac{\pi}{2}}$, this is no longer true for all $n \in \mathbb{N}_{0}$. We denote by $\Delta_{-}(H) \in \mathbb{N}_{0} \cup\{\infty\}$ the number

$\Delta_{-}(H):=\inf \left\{n \in \mathbb{N}_{0}: \exists \omega_{0}, \ldots, \omega_{n} \in \mathbb{C}\right.$ such that $\omega_{0} \neq 0$ and

$$
\left.\sum_{k=0}^{n} \omega_{n-k} \mathcal{I}^{k} \xi_{\phi_{-}(H)+\frac{\pi}{2}} \in L^{2}\left(\left.H\right|_{\left(a, x_{0}\right)}\right)\right\},
$$

where the infimum of the empty set is infinity.

The number $\Delta_{-}(H)$ measures in a certain sense the growth of $H$ towards $a$; for example $\Delta_{-}(H)=0$ means that $H$ is in the limit circle case at $a$ because then all constant vectors are in $L^{2}\left(\left.H\right|_{\left(a, x_{0}\right)}\right)$, i.e. $H$ is integrable at $a$. If $(a, c)$ is an indivisible interval for some $c \in(a, b)$ and $H$ is in the limit point case at $a$, then $\Delta_{-}(H)=1$.

An illustrative toy example occurs in connection with the Bessel equation (this example will be discussed in detail in [LW3]). One can show that

$$
H_{\gamma}(x)=\left(\begin{array}{cc}
x^{\gamma} & 0 \\
0 & x^{-\gamma}
\end{array}\right), \quad x \in(0, \infty),
$$

satisfies $\left(\mathrm{I}_{0}^{-}\right)$and $\left(\mathrm{HS}_{0}^{-}\right)$, and hence $\left(\mathrm{HS}_{-}\right)$, and one has $\Delta_{-}\left(H_{\gamma}\right)=\left\lfloor\frac{\gamma+1}{2}\right\rfloor$.

Assume that $\Delta_{-}(H)<\infty$ and let $x_{0} \in(a, b)$. Then there exist unique absolutely continuous functions $\mathfrak{w}_{l}, l \in \mathbb{N}_{0}$, such that

$$
\begin{array}{ll}
\mathfrak{w}_{0}=\xi_{\phi_{-}}(H)+\frac{\pi}{2}, & \\
\mathfrak{w}_{l} \in L^{2}\left(\left.H\right|_{\left(a, x_{0}\right)}\right), & l \geq \Delta_{-}(H), \\
\mathfrak{w}_{l+1}^{\prime}=J H \mathfrak{w}_{l}, & l \in \mathbb{N}_{0}, \\
\mathfrak{w}_{l}\left(x_{0}\right) \in \operatorname{span}\left\{\xi_{\phi_{-}(H)+\frac{\pi}{2}}\right\}, & l \in \mathbb{N}_{0} .
\end{array}
$$

Note that $\mathfrak{w}_{0}, \ldots, \mathfrak{w}_{\Delta_{-}(H)-1}$ do not belong to $L^{2}\left(\left.H\right|_{\left(a, x_{0}\right)}\right)$. It was shown in $[\mathrm{KW} / \mathrm{IV}$, Lemma $3.12(i)]$ that $\Delta_{-}(H)$ does not depend on the choice of the cutting point $x_{0} \in(a, b)$; the functions $\mathfrak{w}_{l}$ of course do depend on $x_{0}$. The functions $\mathfrak{w}_{0}, \ldots, \mathfrak{w}_{2 \Delta_{-}(H)}$ are used, e.g. to construct regularized boundary values in Theorem 5.1.

For a Hamiltonian $H$ that satisfies $\left(\mathrm{HS}_{+}\right)$a number $\Delta_{+}(H)$ is defined in a similar manner.

\section{e. General Hamiltonians in detail.}

In this subsection we give the definition of a general Hamiltonian as in $[\mathrm{KW} / \mathrm{IV}$, $\S 8]$. This definition is somewhat elaborate, and we give some explanations after the definition. 
2.18 Definition. A general Hamiltonian $\mathfrak{h}$ is a collection of data of the following kind:

(i) $n \in \mathbb{N}_{0}, \sigma_{0}, \ldots, \sigma_{n+1} \in \mathbb{R} \cup\{ \pm \infty\}$ with $\sigma_{0}<\sigma_{1}<\ldots<\sigma_{n+1}$,

(ii) Hamiltonians $H_{i}:\left(\sigma_{i}, \sigma_{i+1}\right) \rightarrow \mathbb{R}^{2 \times 2}$ for $i=0, \ldots, n$, which satisfy (2.6),

(iii) numbers $\ddot{o}_{1}, \ldots, \ddot{o}_{n} \in \mathbb{N} \cup\{0\}$ and $b_{i, 1}, \ldots, b_{i, \ddot{o}_{i}+1} \in \mathbb{R}, i=1, \ldots, n$, with $b_{i, 1} \neq 0$ when $\ddot{o}_{i} \geq 1$

(iv) numbers $d_{i, 0}, \ldots, d_{i, 2 \Delta_{i}-1} \in \mathbb{R}$ where $\Delta_{i}:=\max \left\{\Delta_{+}\left(H_{i-1}\right), \Delta_{-}\left(H_{i}\right)\right\}$ for $i=1, \ldots, n$, (note that $\Delta_{i}$ will be finite by condition (H3) below),

$(v)$ a finite subset $E$ of $\left\{\sigma_{0}, \sigma_{n+1}\right\} \cup \bigcup_{i=0}^{n}\left(\sigma_{i}, \sigma_{i+1}\right)$,

which is assumed to be subject to the following conditions:

(H1) The Hamiltonian $H_{0}$ is in the limit circle case at $\sigma_{0}$. If $n \geq 1$, then $H_{i}$ is in the limit point case at $\sigma_{i}$ for $i=1, \ldots, n$, and at $\sigma_{i+1}$ for $i=0, \ldots, n-1$.

(H2) None of the intervals $\left(\sigma_{i}, \sigma_{i+1}\right), i=1, \ldots, n-1$, is indivisible ${ }^{2}$. If $n \geq 1$ and $H_{n}$ is in the limit point case at $\sigma_{n+1}$, then also $\left(\sigma_{n}, \sigma_{n+1}\right)$ is not indivisible.

(H3) The Hamiltonian $H_{0}$ satisfies $\left(\mathrm{HS}_{+}\right)$if $n \geq 1 ; H_{i}$ satisfies (HS-) and $\left(\mathrm{HS}_{+}\right)$for $i=1, \ldots, n-1$, and $H_{n}$ satisfies (HS-). We have $\Delta_{i}<\infty, i=1, \ldots, n$.

(H4) We have $\phi_{+}\left(H_{i-1}\right)=\phi_{-}\left(H_{i}\right), i=1, \ldots, n$.

(H5) Let $i \in\{1, \ldots, n\}$. If both $H_{i-1}$ and $H_{i}$ end with an indivisible interval towards $\sigma_{i}$, then $d_{1}=0$. If, in addition, $b_{i, 1}=0$, then $d_{0}<0$.

(E1) $\quad \sigma_{0}, \sigma_{n+1} \in E$, and $E \cap\left(\sigma_{i}, \sigma_{i+1}\right) \neq \emptyset$ for $i=1, \ldots, n-1$. If $H_{n}$ is in the limit point case at $\sigma_{n+1}$, then also $E \cap\left(\sigma_{n}, \sigma_{n+1}\right) \neq \emptyset$. Moreover, $E$ contains all endpoints of indivisible intervals of infinite length which lie in $\bigcup_{i=0}^{n}\left(\sigma_{i}, \sigma_{i+1}\right)$.

(E2) No point of $E$ is an inner point of an indivisible interval.

The common value of $\phi_{+}\left(H_{i-1}\right)$ and $\phi_{-}\left(H_{i}\right)$ is denoted by $\phi_{i}$.

The general Hamiltonian $\mathfrak{h}$ is called regular or singular if $H_{n}$ is in the limit circle case or in the limit point case, respectively, at $\sigma_{n+1}$. Moreover, we set

$$
\text { ind }_{-} \mathfrak{h}:=\sum_{i=1}^{n}\left(\Delta_{i}+\left\lfloor\frac{\ddot{o}_{i}}{2}\right\rfloor\right)+\mid\left\{1 \leq i \leq n: \ddot{o}_{i} \text { odd, } b_{i, 1}>0\right\} \mid \text {. }
$$

\footnotetext{
${ }^{2}$ The interval $\left(\sigma_{0}, \sigma_{1}\right)$ may be indivisible.
} 
It is probably helpful to have a more intuitive picture of general Hamiltonians than their precise definition. We may say that a general Hamiltonian models a canonical system on $\left[\sigma_{0}, \sigma_{n+1}\right)$ whose Hamiltonian is allowed to have finitely many inner singularities (these are the points $\sigma_{1}, \ldots, \sigma_{n}$ ), and which is in the limit circle or limit point case at $\sigma_{n+1}$ depending whether $\mathfrak{h}$ is regular or singular. However, $H$ does not behave too badly at its inner singularities in the sense of (H3).

A singularity itself contributes to the canonical system in two ways. The first one is a contribution concentrated inside the singularity; passing the singularity influences a solution, which is modelled by the parameters $\ddot{o}_{i}, b_{i j}$. Actually, elements in the model space in which an operator acts can be considered as a combination of functions and distributions concentrated in the singularities. The parameters $\ddot{o}_{i}$ and $b_{i j}$ are needed for the interplay of the functions and the distributions. The functions themselves have also a singular component, namely a linear combination of $\mathfrak{w}_{0}, \ldots, \mathfrak{w}_{\Delta_{i}-1}$; here the parameters $d_{i j}$ and condition (H4) are used for interface conditions at the singularities.

The set $E$ consists of points that split $\bigcup_{i=0}^{n}\left(\sigma_{i}, \sigma_{i+1}\right)$ into smaller pieces each containing at most one singularity. We can picture the situation as follows $\left(E=\left\{s_{0}, \ldots, s_{N+1}\right\}\right)$ :

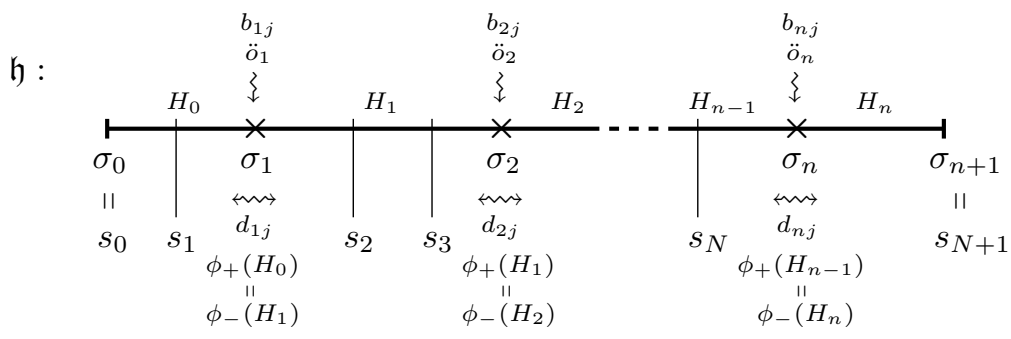

The numbers $d_{i j}$ depend on $s_{k}$ in the sense that, if the $s_{k}$ are moved, then the $d_{i j}$ have to be changed in order to obtain an isomorphic model. Moreover, the number of constants $d_{i j}$ needed for a fixed $i$ depends on the behaviour of $H$ in the neighbourhood of $\sigma_{i}$, namely one needs $2 \Delta_{i}$ constants. On the other hand, the $\ddot{o}_{i}$ and $b_{i j}$ can be chosen independently of the behaviour of $H$ and they do not change when the $s_{k}$ are moved.

With a general Hamiltonian $\mathfrak{h}$ a boundary triple $(\mathcal{P}(\mathfrak{h}), T(\mathfrak{h}), \Gamma(\mathfrak{h}))$ can be associated, whose definition is quite involved; see $[\mathrm{KW} / \mathrm{IV}]$. The Pontryagin space $\mathcal{P}(\mathfrak{h})$, which has negative index ind $-\mathfrak{h}$, is obtained as a completion and therefore not very accessible. For a special case we shall consider a more concrete realization of the model space and the maximal relation $T(\mathfrak{h})$. In $[\mathrm{KW} / \mathrm{IV}$, Definition 8.5] a mapping $\psi(\mathfrak{h})$ was defined that maps an element $F$ in $\mathcal{P}(\mathfrak{h})$ onto some measurable function defined on $\bigcup_{i=0}^{n}\left(\sigma_{i}, \sigma_{i+1}\right)$, which represents the 'function part' of $F$.

Analogously to the classical positive definite case where the fundamental solution $W_{H}$ can be associated with the Hamiltonian $H$ one can define a 'fundamental solution' $\omega_{\mathfrak{h}}$ for an indefinite Hamiltonian $\mathfrak{h}$ where $\omega_{\mathfrak{h}}(x ; z)$ is a $2 \times 2$ matrix for every $x \in\left[\sigma_{0}, \sigma_{1}\right) \cup \bigcup_{i=1}^{n}\left(\sigma_{i}, \sigma_{i+1}\right)$ and $z \in \mathbb{C}$. For fixed $z$ it satisfies the differential equation (2.7) on every interval between the singularities and one has $\omega_{\mathfrak{h}}\left(\sigma_{0} ; z\right)=I$ for every $z \in \mathbb{C}$. Moreover, for fixed $x, \omega_{\mathfrak{h}}(x ; \cdot)$ is an 
entire function belonging to the class $\mathcal{M}_{<\infty}$ such that $x \mapsto \operatorname{ind}_{-} \omega_{\mathfrak{h}}(x ; \cdot)$ is non-decreasing, constant between the singularities and

$$
\max \left\{\operatorname{ind}_{-} \omega_{\mathfrak{h}}(x ; \cdot): x \in\left[\sigma_{0}, \sigma_{1}\right) \cup \bigcup_{i=1}^{n}\left(\sigma_{i}, \sigma_{i+1}\right)\right\}=\text { ind }_{-} \mathfrak{h} .
$$

The definition of $\omega_{\mathfrak{h}}$ is quite involved, in particular, how one can jump over a singularity; for details see $\left[\mathrm{KW} / \mathrm{V}\right.$, Definition 5.3]. The function $\omega_{\mathfrak{h}}$ is called maximal chain if $\mathfrak{h}$ is singular and finite maximal chain if $\mathfrak{h}$ is regular.

In the case when the indefinite Hamiltonian $\mathfrak{h}$ is singular one can define the Titchmarsh-Weyl coefficient $q_{\mathfrak{h}}$ in a similar way as in the positive definite case:

$$
q_{\mathfrak{h}}(z):=\lim _{x \nearrow \sigma_{n+1}} \omega_{\mathfrak{h}}(x ; z) \star \tau, \quad z \in \mathbb{C} \backslash \mathbb{R},
$$

with $\tau \in \mathbb{R} \cup\{\infty\}$; the limit exists locally uniformly on $\mathbb{C} \backslash \mathbb{R}$ with respect to the chordal metric on the Riemann sphere $\overline{\mathbb{C}}$, defines a meromorphic function on $\mathbb{C} \backslash \mathbb{R}$, and the limit is independent of $\tau$. The Titchmarsh-Weyl coefficient $q_{\mathfrak{h}}$ belongs to the class $\mathcal{N}_{\kappa}$ where $\kappa=$ ind $_{-} \mathfrak{h}$; see [KW/VI, Theorem 1.4]. At the interior singularities $\sigma_{1}, \ldots, \sigma_{n}$ one can define intermediate Weyl coefficients, $q_{\mathfrak{h}, \sigma_{i}}$, by

$$
q_{\mathfrak{h}, \sigma_{i}}(z):=\lim _{x \rightarrow \sigma_{i}} \omega_{\mathfrak{h}}(x ; z) \star \tau, \quad z \in \mathbb{C} \backslash \mathbb{R}, \quad i=1, \ldots, n,
$$

again for $\tau \in \mathbb{R} \cup\{\infty\}$. It is an essential and non-trivial fact that the limits from both sides of the singularity coincide; see [KW/III, Theorem 5.6].

2.19. Splitting of general Hamiltonians. On working with general Hamiltonians one often uses a splitting-and-pasting process. Let us briefly recall how a general Hamiltonian $\mathfrak{h}$ can be split into smaller parts. Let $\mathfrak{h}$ be given by the data

$$
\sigma_{0}, \ldots, \sigma_{n+1}, \quad H_{0}, \ldots, H_{n}, \quad \ddot{o}_{i}, b_{i j}, d_{i j}, \quad i=1, \ldots, n, \quad E,
$$

and let $s \in \bigcup_{i=0}^{n}\left(\sigma_{i}, \sigma_{i+1}\right)$ be not inner point of an indivisible interval. Then a splitting of $\mathfrak{h}$ into two general Hamiltonians, $\mathfrak{h}_{\uparrow s}$ and $\mathfrak{h}_{s \triangleright}$, can be defined. Namely, $\mathfrak{h}_{\uparrow s}$ is given by the data ( $k$ is such that $\left.\sigma_{k}<s<\sigma_{k+1}\right)$

$$
\begin{gathered}
\sigma_{0}, \ldots, \sigma_{k}, s, \quad H_{0}, \ldots,\left.H_{k}\right|_{\left(\sigma_{k}, s\right)}, \\
\ddot{o}_{i}, b_{i j}, d_{i j}, \quad i=1, \ldots, k-1, \ddot{o}_{k}, b_{k j}, \tilde{d}_{k j}, \quad\left(E \cap\left[\sigma_{0}, s\right]\right) \cup\{s\},
\end{gathered}
$$

where the numbers $\tilde{d}_{k j}$ depend on the location of $s$, cf. [KW/V, Definition 3.47]. The general Hamiltonian $\mathfrak{h}_{s \gg}$ is given by data

$$
\begin{gathered}
s, \sigma_{k+1}, \ldots, \sigma_{n+1},\left.\quad H_{k}\right|_{\left(s, \sigma_{k+1}\right)}, H_{k+1}, \ldots, H_{n}, \\
\ddot{o}_{k+1}, b_{k+1, j}, \tilde{d}_{k+1, j}, \quad \ddot{o}_{i}, b_{i j}, d_{i j}, \quad i=k+2, \ldots, n, \quad\left(E \cap\left[s, \sigma_{n+1}\right]\right) \cup\{s\},
\end{gathered}
$$

where again $\tilde{d}_{k+1, j}$ depend on the location of $s$.

If $s=\sigma_{k}$, we let $\mathfrak{h}_{\mathfrak{t} s}$ be given by

$$
\begin{aligned}
\mathfrak{h}_{\text {ฯs }}: \quad & \sigma_{0}, \ldots, \sigma_{k}, \quad H_{0}, \ldots, H_{k-1}, \\
& \ddot{o}_{i}, b_{i j}, d_{i j}, i=1, \ldots, k-1, \quad\left(E \cap\left[\sigma_{0}, \sigma_{k}\right]\right) \cup\left\{\sigma_{k}\right\} .
\end{aligned}
$$


These notions have been studied in detail in $[\mathrm{KW} / \mathrm{V}]$. In particular, it was shown that splitting of general Hamiltonians gives rise to splitting of operator models, and it is compatible with fundamental solutions. In particular, the model space $\mathcal{P}(\mathfrak{h})$ is isomorphic to $\mathcal{P}\left(\mathfrak{h}_{\uparrow s}\right)[\dot{+}] \mathcal{P}\left(\mathfrak{h}_{s \triangleright}\right)$; where $[\dot{+}]$ denotes a direct and orthogonal sum in the Pontryagin space $\mathcal{P}(\mathfrak{h})$. Elements in $T(\mathfrak{h})$ can be

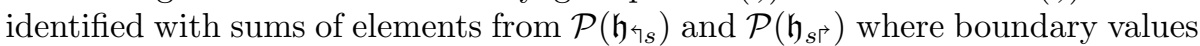
of the latter have to coincide at $s$. For details see $[\mathrm{KW} / \mathrm{V}, \S 3 . \mathrm{c} / \mathrm{e}, \S 5 . \mathrm{d}]$.

For $\mathcal{P}=\mathcal{P}\left(\mathfrak{h}_{\uparrow s}\right)$ an 'isomorphism $\Xi$ ' as in Remark 2.12 can be constructed. It is an isomorphism from $\mathcal{P}\left(\mathfrak{h}_{\text {}}\right)$ onto $\mathfrak{K}\left(\omega_{\mathfrak{h}}(s)\right)$ and denoted by $\Xi_{s}$.

\section{f. A boundary triple for a certain regular general Hamiltonian.}

As mentioned above, the construction of the boundary triple $(\mathcal{P}(\mathfrak{h}), T(\mathfrak{h}), \Gamma(\mathfrak{h}))$ is quite complicated and not easy to use. For singular general Hamiltonians with one singularity a more explicit form of this model, i.e. a boundary triple $(\stackrel{\infty}{\mathcal{P}}(\mathfrak{h}), \stackrel{\infty}{T}(\mathfrak{h}), \stackrel{\infty}{\Gamma}(\mathfrak{h}))$ that is isomorphic to the original one, was constructed in [LW2]. This form turned out to be more convenient for some purposes and is more intuitive in the sense that $\mathcal{P}(\mathfrak{h})$ is a finite-dimensional extension of a natural function space and $T(\mathfrak{h})$ is a finite-dimensional perturbation/extension of a natural differential operator in this function space.

In the present paper we use a variant of this model for regular general Hamiltonians of the class $\mathfrak{H}_{\frac{\pi}{2}}$.

2.20. Particular form of $\mathfrak{h}$. Let $\mathfrak{h} \in \mathfrak{H}_{\frac{\pi}{2}}$ be given by the data

$$
\sigma_{0}, \sigma_{1}, \sigma_{2}, \quad H_{0}, H_{1}, \quad \ddot{o}, b_{j}, d_{j}, \quad E,
$$

such that $\sigma_{1}$ is not left endpoint of an indivisible interval, $\sigma_{2}<\infty, E=\left\{\sigma_{0}, \sigma_{2}\right\}$, and $b_{\ddot{o}+1}=0$.

Note that $\phi_{+}\left(H_{0}\right)=0$ because of $\left(\mathrm{gH}_{\frac{\pi}{2}}\right)$ and therefore also $\phi_{-}\left(H_{1}\right)=0$. Since $\Delta_{+}\left(H_{0}\right)=1$, we have $\Delta:=\Delta_{1}=\Delta_{-}\left(H_{1}\right)$. Further, denote by $H$ the function defined as $H_{0}$ on $\left(\sigma_{0}, \sigma_{1}\right)$ and as $H_{1}$ on $\left(\sigma_{1}, \sigma_{2}\right)$ and let $L^{2}(H)=$ $L^{2}\left(H_{0}\right) \oplus L^{2}\left(H_{1}\right)$. Moreover, let $\mathfrak{w}_{k}, k \in \mathbb{N}_{0}$, be the unique absolutely continuous functions defined on $\left[\sigma_{0}, \sigma_{1}\right) \cup\left(\sigma_{1}, \sigma_{2}\right]$ with the properties that

$$
\begin{aligned}
& \mathfrak{w}_{0}=\left(\begin{array}{l}
0 \\
1
\end{array}\right), \quad\left(\mathfrak{w}_{k}\right)^{\prime}=J H \mathfrak{w}_{k-1}, k \geq 1, \\
& \mathfrak{w}_{k}\left(\sigma_{0}\right), \mathfrak{w}_{k}\left(\sigma_{2}\right) \in \operatorname{span}\left\{\left(\begin{array}{l}
0 \\
1
\end{array}\right)\right\}, k \in \mathbb{N}_{0}, \\
& \mathfrak{w}_{k} \in L^{2}\left(\left.H\right|_{\left(\sigma_{0}, \sigma_{1}\right) \cup\left(\sigma_{1}, \sigma_{2}\right)}\right), k \geq \Delta ;
\end{aligned}
$$

cf. (2.12). Then $\left\{\mathfrak{w}_{0}, \ldots, \mathfrak{w}_{\Delta-1}\right\}$ is linearly independent modulo $L^{2}(H)$ by $[\mathrm{KW} / \mathrm{IV}$, Lemma 3.6]. We define the function space

$$
L_{\Delta}^{2}(H):=L^{2}(H) \dot{+} \operatorname{span}\left\{\mathfrak{w}_{k}: k=0, \ldots, \Delta-1\right\}
$$

and the differential operator $T_{\Delta, \max }(H)$ (or, more precisely, its graph) by

$$
\begin{array}{r}
T_{\Delta, \max }(H):=\left\{(f ; g) \in L_{\Delta}^{2}(H) \times L_{\Delta}^{2}(H): \exists \hat{f}\right. \text { absolutely continuous } \\
\text { representative of } \left.f \text { s.t. } \hat{f}^{\prime}=J H g\right\} .
\end{array}
$$


Now we can define a boundary triple $(\stackrel{\infty}{\mathcal{P}}(\mathfrak{h}), \stackrel{\infty}{T}(\mathfrak{h}), \stackrel{\infty}{\Gamma}(\mathfrak{h}))$ which will turn out to be isomorphic to the original model $(\mathcal{P}(\mathfrak{h}), T(\mathfrak{h}), \Gamma(\mathfrak{h}))$.

2.21 Definition. Let $\mathfrak{h}$ be a general Hamiltonian of the form described in 2.20. Then $\stackrel{\infty}{\mathcal{P}}(\mathfrak{h}), \stackrel{\infty}{T}(\mathfrak{h})$ and $\stackrel{\infty}{\Gamma}(\mathfrak{h})$ are defined as follows.

The base space $\stackrel{\infty}{\mathcal{P}}(\mathfrak{h})$. Set

$$
\stackrel{\infty}{\mathcal{P}}(\mathfrak{h}):=L_{\Delta}^{2}(H) \times \mathbb{C}^{\Delta} \times \mathbb{C}^{\ddot{o}} .
$$

Elements of $\stackrel{\oplus}{\mathcal{P}}(\mathfrak{h})$ are generically written as $F=(f ; \xi, \alpha)$ with $\xi=\left(\xi_{j}\right)_{j=0}^{\Delta-1}$ and $\alpha=\left(\alpha_{j}\right)_{j=1}^{\ddot{o}}$ or $G=(g ; \eta, \beta)$ with $\eta=\left(\eta_{j}\right)_{j=0}^{\Delta-1}$ and $\beta=\left(\beta_{j}\right)_{j=1}^{\ddot{o}}$. Note that the case $\ddot{o}=0$ is allowed.

By the definition of $L_{\Delta}^{2}(H)$ and the fact that $\mathfrak{w}_{0}, \ldots, \mathfrak{w}_{\Delta-1}$ are linearly independent modulo $L^{2}(H)$, for given $f, g \in L_{\Delta}^{2}(H)$, there exist unique scalars $\lambda_{l}, \mu_{l}, l=0, \ldots, \Delta-1$, such that

$$
\tilde{f}:=f-\sum_{l=0}^{\Delta-1} \lambda_{l} \mathfrak{w}_{l}, \quad \tilde{g}:=g-\sum_{l=0}^{\Delta-1} \mu_{l} \mathfrak{w}_{l} \in L^{2}(H) .
$$

The inner product on $\stackrel{\infty}{\mathcal{P}}(\mathfrak{h})$. If $\ddot{o}>0$, define numbers $c_{1}, \ldots, c_{\ddot{o}}$ recursively by

$$
c_{1} b_{1}=-1 ; \quad \sum_{j=1}^{k} c_{j} b_{k-j}=0, \quad k=2, \ldots, \ddot{o} .
$$

On $\stackrel{\infty}{\mathcal{P}}(\mathfrak{h})$ an inner product is defined by

$$
[F, G]=(\tilde{f}, \tilde{g})_{L^{2}(H)}+\sum_{k=0}^{\Delta-1} \lambda_{k} \overline{\eta_{k}}+\sum_{k=0}^{\Delta-1} \xi_{k} \overline{\mu_{k}}+\sum_{k, l=1}^{\ddot{o}} c_{k+l-\ddot{o}} \alpha_{k} \overline{\beta_{l}}
$$

for $F$ and $G$ as above.

The maximal relation $\stackrel{\infty}{T}(\mathfrak{h})$. Let $F=(f ; \xi, \alpha), G=(g ; \eta, \beta) \in \stackrel{\infty}{\mathcal{P}}(\mathfrak{h})$ and $\lambda_{l}, \mu_{l}$ be the scalars as above. Then $(F ; G) \in \stackrel{\infty}{T}(\mathfrak{h})$ if and only if

(i) $(f ; g) \in T_{\Delta, \max }(H)$

(which implies that $\mu_{k}=\lambda_{k+1}, k=0, \ldots, \Delta-2$ );

(ii) for all $k=0, \ldots, \Delta-2$,

$$
\xi_{k}=\eta_{k+1}+\frac{1}{2} \mu_{\Delta-1} d_{\Delta+k}+\frac{1}{2} \lambda_{0} d_{k}+\left(\mathfrak{w}_{k+1}\left(\sigma_{2}\right)\right)_{2} f\left(\sigma_{2}\right)_{1} ;
$$

(iii)

$$
\begin{aligned}
\xi_{\Delta-1}=\int_{\sigma_{1}}^{\sigma_{2}}\left(\mathfrak{w}_{\Delta}\right)^{*} H \tilde{g}+\frac{1}{2} \sum_{l=0}^{\Delta-1} \lambda_{l} d_{l+\Delta-1}+\mu_{\Delta-1} d_{2 \Delta-1} & \\
& +\left(\mathfrak{w}_{\Delta}\left(\sigma_{2}\right)\right)_{2} f\left(\sigma_{2}\right)_{1}- \begin{cases}\beta_{1}, & \ddot{o}>0, \\
0, & \ddot{o}=0 ;\end{cases}
\end{aligned}
$$


(iv) if $\ddot{o}>0$, then

$$
\alpha_{j}=\mu_{\Delta-1} b_{\ddot{o}-j+1}+ \begin{cases}\beta_{j+1}, & j=1, \ldots, \ddot{o}-1, \\ 0, & j=\ddot{o} .\end{cases}
$$

Here $f\left(\sigma_{2}\right)$ denotes the value of the unique absolutely continuous representative with $f^{\prime}=J H g$ (remember here that $\left(\sigma_{1}, \sigma_{2}\right)$ is not indivisible).

The boundary operator $\stackrel{\infty}{\Gamma}(\mathfrak{h})$. The boundary relation is actually an operator. Let $(F ; G) \in \stackrel{\infty}{T}(\mathfrak{h})$, write $F=(f ; \xi, \alpha), G=(g ; \eta, \beta)$ and let $\lambda_{l}, \mu_{l}$ be as in (2.18). Then

$$
\stackrel{\infty}{\Gamma}(\mathfrak{h})(F ; G):=\left(\left(\begin{array}{c}
\eta_{0}+f\left(\sigma_{2}\right)_{1}+\frac{1}{2} \sum_{l=0}^{\Delta-1} \mu_{l} d_{l} \\
\lambda_{0}
\end{array}\right) ; f\left(\sigma_{2}\right)\right) .
$$

The two components of $\stackrel{\infty}{\Gamma}(\mathfrak{h})(F ; G)$ can be interpreted as boundary values at the left and right endpoints $\sigma_{0}$ and $\sigma_{2}$, respectively; we denote these by $\pi_{l} \circ$ $\stackrel{\infty}{\Gamma}(\mathfrak{h})(F ; G)$ and $\pi_{r} \circ \stackrel{\infty}{\Gamma}(\mathfrak{h})(F ; G)$. The two components of $\pi_{l} \circ \Gamma_{\Gamma}^{\infty}(\mathfrak{h})(F ; G)$ are denoted by $\pi_{l, 1} \circ \stackrel{\infty}{\Gamma}(\mathfrak{h})(F ; G)$ and $\pi_{l, 2} \circ \Gamma^{\infty}(\mathfrak{h})(F ; G)$.

2.22 Proposition. Let $\mathfrak{h}$ be a general Hamiltonian of the form 2.20. Then the boundary triples $(\mathcal{P}(\mathfrak{h}), T(\mathfrak{h}), \Gamma(\mathfrak{h}))$ and $(\stackrel{\infty}{\mathcal{P}}(\mathfrak{h}), \stackrel{\infty}{T}(\mathfrak{h}), \stackrel{\infty}{\Gamma}(\mathfrak{h}))$ are isomorphic.

Proof. Let $\tilde{\mathfrak{h}}$ be the general Hamiltonian that is given by the data

$$
\begin{gathered}
\sigma_{0}, \sigma_{1}, \infty, \quad \tilde{H}_{0}:=H_{0}, \tilde{H}_{1}(t):= \begin{cases}H_{1}(t), & t \in\left(\sigma_{1}, \sigma_{2}\right), \\
I, & t \in\left[\sigma_{2}, \infty\right),\end{cases} \\
\ddot{o}, b_{j}, d_{j}, \quad E:=\left\{\sigma_{0}, \sigma_{2}, \infty\right\} .
\end{gathered}
$$

Then $\tilde{\mathfrak{h}}$ is of the form considered in [LW2]. Moreover, the boundary triple $\left(\stackrel{\infty}{\mathcal{P}}_{\sigma_{2}}(\tilde{\mathfrak{h}}), \stackrel{\infty}{T}_{\sigma_{2}}(\tilde{\mathfrak{h}}), \stackrel{\infty}{\Gamma}_{\sigma_{2}}(\tilde{\mathfrak{h}})\right)$, which was defined in [LW2, Definition 2.14], is isomorphic to $(\mathcal{P}(\tilde{\mathfrak{h}}), T(\tilde{\mathfrak{h}}), \Gamma(\tilde{\mathfrak{h}}))$ by definition, and has been described explicitly in [LW2, Theorem 2.15]. Note, however, that there is a typo in [LW2, Theorem $2.15(\mathrm{v})]$, namely, there should be a plus sign instead of a minus sign in front of $\mu_{\Delta-1}$, as in (2.20). Let ${\stackrel{\infty}{\iota_{\sigma_{2}}}}^{\infty}:=i_{\sigma_{2}} \circ \iota_{\sigma_{2}}$ be the isomorphism from $\mathcal{P}(\tilde{\mathfrak{h}})$ onto $\stackrel{\infty}{\mathcal{P}}_{\sigma_{2}}(\tilde{\mathfrak{h}})$ where $i_{\sigma_{2}}$ and $\iota_{\sigma_{2}}$ were defined in [LW2, §2].

The following diagram is commutative

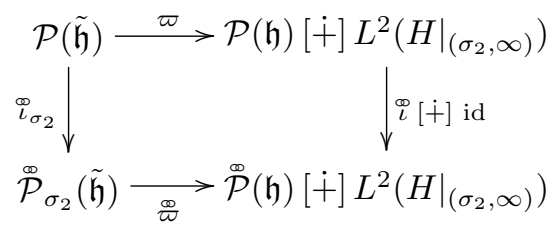

where $\varpi$ was defined in [KW/IV, Proposition 8.11], $\iota^{\infty}:=i \circ \iota$ with

$$
\iota: \mathcal{P}(\mathfrak{h}) \rightarrow L^{2}(H) \times \mathbb{C}^{\Delta} \times \mathbb{C}^{\Delta} \times \mathbb{C}^{\ddot{o}}
$$


as in $[\mathrm{KW} / \mathrm{IV},(4.9)$ and (4.10)] and

$$
\begin{gathered}
i: L^{2}(H) \times \mathbb{C}^{\Delta} \times \mathbb{C}^{\Delta} \times \mathbb{C}^{\ddot{o}} \rightarrow L_{\Delta}^{2}(H) \times \mathbb{C}^{\Delta} \times \mathbb{C}^{\ddot{o}} \\
(f ; \xi, \lambda, \alpha) \mapsto\left(f+\sum_{k=0}^{\Delta-1} \lambda_{k} \mathfrak{w}_{k} ; \xi, \alpha\right),
\end{gathered}
$$

and $\stackrel{\infty}{\varpi}$ acts as

$$
\varpi:\left.F \mapsto\left(\left.f\right|_{\left(\sigma_{1}, \sigma_{1}\right) \cup\left(\sigma_{1}, \sigma_{2}\right)} ; \xi, \alpha\right)[\dot{+}] f\right|_{\left(\sigma_{2}, \infty\right)}, \quad F=(f ; \xi, \alpha) \in \stackrel{\infty}{\mathcal{P}}_{\sigma_{2}}(\tilde{\mathfrak{h}}) .
$$

The definition of $\stackrel{\infty}{\mathcal{P}}(\mathfrak{h})$ and [LW2, Theorem 2.15] show that

$$
\begin{aligned}
& (\tilde{F} ; \tilde{G}) \in \mathscr{\Phi}_{\sigma_{2}}(\tilde{\mathfrak{h}}) \Longleftrightarrow \\
& \quad\left(\tilde{F}_{\uparrow \sigma_{2}} ; \tilde{G}_{\uparrow \sigma_{2}}\right) \in \stackrel{\infty}{T}(\mathfrak{h}),\left(\left.\tilde{f}\right|_{\left(\sigma_{2}, \infty\right)} ;\left.\tilde{g}\right|_{\left(\sigma_{2}, \infty\right)}\right) \in T_{\max }\left(\left.H\right|_{\left(\sigma_{2}, \infty\right)}\right), \\
& \quad \tilde{f} \text { continuous at } \sigma_{2}
\end{aligned}
$$

where $\tilde{F}=(\tilde{f} ; \xi, \alpha), \tilde{G}=(\tilde{g} ; \eta, \beta)$ and $\tilde{F}_{\urcorner_{\sigma_{2}}}:=\left(\left.\tilde{f}\right|_{\left(\sigma_{1}, \sigma_{1}\right) \cup\left(\sigma_{1}, \sigma_{2}\right)} ; \xi, \alpha\right)$. In this case,

$$
\begin{aligned}
& \pi_{l} \circ \stackrel{\infty}{\Gamma}(\mathfrak{h})\left(\tilde{F}_{\eta_{\sigma_{2}}} ; \tilde{G}_{\eta_{\sigma_{2}}}\right)=\pi_{l} \circ \stackrel{\infty}{\Gamma}_{\sigma_{2}}(\tilde{\mathfrak{h}})(\tilde{F} ; \tilde{G}),
\end{aligned}
$$

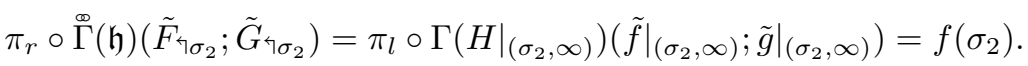

Choose $\left(f_{1} ; g_{1}\right),\left(f_{2} ; g_{2}\right) \in T_{\max }\left(\left.H\right|_{\left(\sigma_{2}, \infty\right)}\right)$ with $f_{1}\left(\sigma_{2}\right)=\left(\begin{array}{l}1 \\ 0\end{array}\right)$ and $f_{2}\left(\sigma_{2}\right)=\left(\begin{array}{l}0 \\ 1\end{array}\right)$.

Now let $F=(f ; \xi, \alpha), G=(g ; \eta, \beta) \in \stackrel{\infty}{\mathcal{P}}(\mathfrak{h})$ be given and assume that $(F ; G) \in \stackrel{\infty}{T}(\mathfrak{h})$. Then we set

$$
\begin{aligned}
& \tilde{f}:=f \chi_{\left(\sigma_{0}, \sigma_{1}\right) \cup\left(\sigma_{1}, \sigma_{2}\right)}+f\left(\sigma_{2}\right)_{1} f_{1}+f\left(\sigma_{2}\right)_{2} f_{2}, \\
& \tilde{g}:=g \chi_{\left(\sigma_{0}, \sigma_{1}\right) \cup\left(\sigma_{1}, \sigma_{2}\right)}+f\left(\sigma_{2}\right)_{1} g_{1}+f\left(\sigma_{2}\right)_{2} g_{2},
\end{aligned}
$$

and

$$
\tilde{F}:=(\tilde{f} ; \xi, \alpha), \tilde{G}:=(\tilde{g} ; \eta, \beta) .
$$

By $(2.23)$, we have $(\tilde{F} ; \tilde{G}) \in \stackrel{\infty}{T}_{\sigma_{2}}(\tilde{\mathfrak{h}})$. Hence

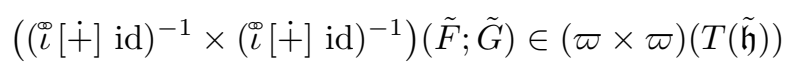

by the commutativity of the diagram in (2.22), and it follows that $(\stackrel{\infty}{i} \times$ $\stackrel{\infty}{\iota})^{-1}(F ; G) \in T(\mathfrak{h})$. This shows that $\left(\stackrel{\Phi}{l}^{-1} \times \mathscr{\Phi}^{-1}\right) \stackrel{\infty}{\mathscr{\infty}}(\mathfrak{h}) \subseteq T(\mathfrak{h})$. Moreover,

$$
\Gamma(\mathfrak{h})\left(\left(\left(^{\infty} \iota^{-1} \times \stackrel{\complement}{\iota}^{-1}\right)(F ; G)\right)=\stackrel{\infty}{\Gamma}(\mathfrak{h})(F ; G)\right.
$$

by (2.24). The reverse inclusion $\left({ }^{\infty} \stackrel{\infty}{l}^{-1} \times \stackrel{\Phi}{\iota}^{-1}\right) \stackrel{\Phi}{T}(\mathfrak{h}) \supseteq T(\mathfrak{h})$ is seen in a similar way. Hence the boundary triples $(\mathcal{P}(\mathfrak{h}), T(\mathfrak{h}), \Gamma(\mathfrak{h}))$ and $(\stackrel{\infty}{\mathcal{P}}(\mathfrak{h}), \stackrel{\infty}{T}(\mathfrak{h}), \stackrel{\infty}{\Gamma}(\mathfrak{h}))$ are isomorphic.

2.23 Remark. If one defines a mapping $\stackrel{\infty}{\psi}(\mathfrak{h})$ by $\stackrel{\infty}{\psi}(\mathfrak{h}) F:=f$ where $F=(f ; \xi ; \alpha)$

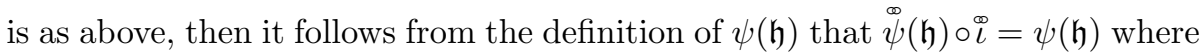
$\iota$ is as in the previous proof. 


\section{g. Rotation isomorphisms.}

We consider general Hamiltonians of the class $\mathfrak{H}_{\alpha}$ on the one hand, and functions from the class $\mathcal{N}_{<\infty}^{(\cot \alpha)}$ for $\alpha \in[0, \pi)$ on the other. A rotation isomorphism is a tool which allows us to restrict explicit proofs to one particular value of $\alpha$. Such isomorphisms exist on all levels (Hamiltonians, functions, fundamental solutions etc.), and the corresponding constructions are compatible with each other.

Let us now give the definitions, cf. $[\mathrm{KW} / \mathrm{V}$, Definition 2.4]. For $\alpha \in \mathbb{R}$ set

$$
N_{\alpha}:=\left(\begin{array}{cc}
\cos \alpha & \sin \alpha \\
-\sin \alpha & \cos \alpha
\end{array}\right) .
$$

2.24 Definition. Let $\alpha \in \mathbb{R}$.

(i) If $M$ is a $2 \times 2$-matrix, set

$$
\circlearrowleft_{\alpha} M:=N_{\alpha} M N_{\alpha}^{-1}
$$

(ii) Application of ' $\sigma_{\alpha}$ ' to matrix functions is always understood pointwise, e.g.

$$
\left(\circlearrowleft_{\alpha} H\right)(x):=\circlearrowleft_{\alpha}(H(x)), \quad\left(\circlearrowleft_{\alpha} \omega_{\mathfrak{h}}\right)(x ; z):=\circlearrowleft_{\alpha}\left(\omega_{\mathfrak{h}}(x ; z)\right) .
$$

(iii) If $q(z)$ is a scalar function, set

$$
\circlearrowleft_{\alpha} q(z):=N_{\alpha} \star q(z) .
$$

(iv) If $\mathfrak{h}$ is a general Hamiltonian given by the data

$$
\sigma_{i}, \quad H_{i}, \quad \ddot{o}_{i}, b_{i j}, d_{i j}, \quad E,
$$

let $\circlearrowleft_{\alpha} \mathfrak{h}$ be the general Hamiltonian given by

$$
\sigma_{i}, \quad \circlearrowleft_{\alpha} H_{i}, \quad \ddot{o}_{i}, b_{i j}, d_{i j}, \quad E .
$$

Some practical computational rules have been collected after $[\mathrm{KW} / \mathrm{V}$, Definition 2.4] and in [KW/V, (2.16), (2.24), (3.1)].

Some compatibility properties of the operations ' $\circlearrowleft_{\alpha}$ ' on the different levels are, for example,

$$
\omega_{\circlearrowleft_{\alpha} \mathfrak{h}}=\circlearrowleft_{\alpha} \omega_{\mathfrak{h}} \quad \text { and } \quad q_{\circlearrowleft_{\alpha} \mathfrak{h}}=\circlearrowleft_{\alpha} q_{\mathfrak{h}},
$$

cf. [KW/V, Lemma 5.14].

2.25 Lemma. Let $H$ be a Hamiltonian defined on an interval $(a, b)$, let $\beta \in \mathbb{R}$, and set $\tilde{H}:=\circlearrowleft_{\beta} H$. Then $H$ satisfies $\left(\mathrm{HS}_{-}\right)$if and only if $\tilde{H}$ does. In this case, $\phi_{-}(\tilde{H})=\phi_{-}(H)-\beta$.

Assume that $H$ (and thus also $\tilde{H}$ ) satisfies $\left(\mathrm{HS}_{-}\right)$. Then $\Delta_{-}(\tilde{H})=_{-} \Delta_{-}(H)$. If $\Delta_{-}(H)$ (and thus also $\left.\Delta_{-}(\tilde{H})\right)$ is finite, and $\mathfrak{w}_{l}, \tilde{\mathfrak{w}}_{l}, l \in \mathbb{N}_{0}$, denote the corresponding functions (2.12), then $\tilde{\mathfrak{w}}_{l}=N_{\beta} \mathfrak{w}_{l}, l \in \mathbb{N}_{0}$. 
Proof. We have $f^{T} H f=\left(N_{\beta} f\right)^{T}\left(\circlearrowleft_{\beta} H\right)\left(N_{\beta} f\right)$, and hence the map $f \mapsto N_{\beta} f$ is an isometric isomorphism from $L^{2}\left(\left.H\right|_{\left(a, x_{0}\right)}\right)$ onto $L^{2}\left(\left.\tilde{H}\right|_{\left(a, x_{0}\right)}\right)$. In particular,

$$
\left(\mathrm{I}_{\alpha}^{-}\right) \text {for } H \Longleftrightarrow\left(\mathrm{I}_{\alpha-\beta}^{-}\right) \text {for } \circlearrowleft_{\beta} H \text {. }
$$

As in the definition of the condition $\left(\mathrm{HS}_{\alpha}^{-}\right)$let $M$ be an anti-derivative of $H$ and $\tilde{M}$ an anti-derivative of $\tilde{H}$. Then $\tilde{M}=\circlearrowleft_{\beta} M$, and hence

$$
\xi_{\alpha+\frac{\pi}{2}}^{T} M \xi_{\alpha+\frac{\pi}{2}}=\left(N_{\beta} \xi_{\alpha+\frac{\pi}{2}}\right)^{T}\left(\circlearrowleft_{\beta} M\right)\left(N_{\beta} \xi_{\alpha+\frac{\pi}{2}}\right)=\xi_{(\alpha-\beta)+\frac{\pi}{2}}^{T} \tilde{M} \xi_{(\alpha-\beta)+\frac{\pi}{2}} .
$$

Thus also

$$
\left(\mathrm{HS}_{\alpha}^{-}\right) \text {for } H \Longleftrightarrow\left(\mathrm{HS}_{\alpha-\beta}^{-}\right) \text {for } \circlearrowleft_{\beta} H
$$

and $\phi_{-}(\tilde{H})=\phi_{-}(H)-\beta$. Assume that $H$ satisfies $\left(\mathrm{HS}_{-}\right)$and that $\Delta_{-}(H)<\infty$. Let $\mathfrak{w}_{l}, l \in \mathbb{N}_{0}$, be the unique functions with (2.12), and set

$$
\mathfrak{v}_{l}:=N_{\beta} \mathfrak{w}_{l}, \quad l \in \mathbb{N}_{0} .
$$

Then

$$
\begin{aligned}
& \mathfrak{v}_{0}=\xi_{\phi_{-}(H)+\frac{\pi}{2}-\beta}=\xi_{\phi_{-}(\tilde{H})+\frac{\pi}{2}}, \\
& \mathfrak{v}_{l} \in L^{2}\left(\left.\tilde{H}\right|_{\left(a, x_{0}\right)}\right), \quad l \geq \Delta_{-}(H), \\
& \mathfrak{v}_{l}\left(x_{0}\right) \in \operatorname{span}\left\{\xi_{\phi_{-}(H)+\frac{\pi}{2}-\beta}\right\}, \quad l \in \mathbb{N}_{0},
\end{aligned}
$$

and

$$
\mathfrak{v}_{l+1}^{\prime}=N_{\beta} \mathfrak{w}_{l+1}^{\prime}=N_{\beta} J H \mathfrak{w}_{l}=J N_{\beta} H N_{\beta}^{-1}\left(N_{\beta} \mathfrak{w}_{l}\right)=J \tilde{H} \mathfrak{v}_{l} .
$$

It follows that $\Delta_{-}(\tilde{H}) \leq \Delta_{-}(H)$, and hence, in particular, $\Delta_{-}(\tilde{H})<\infty$. Applying the above argument and the rotation $\circlearrowleft_{-\beta}$ starting from $\tilde{H}$ we obtain $\Delta_{-}(H) \leq \Delta_{-}(\tilde{H})$. Moreover, we see that the functions $\mathfrak{v}_{l}, l \in \mathbb{N}_{0}$, satisfy (2.12) for $\tilde{H}$.

In the present context it is important to know how the classes $\mathfrak{H}_{\alpha}$ and $\mathcal{N}_{<\infty}^{(\cot \alpha)}$, respectively, transform under application of a rotation isomorphism.

2.26 Lemma. Let $\mathfrak{h} \in \mathfrak{H}_{\alpha}$, and let $\beta \in \mathbb{R}$. Then $\circlearrowleft_{\beta} \mathfrak{h} \in \mathfrak{H}_{\alpha-\beta}$.

Proof. It is obvious from the definition of $\circlearrowleft_{\beta} \mathfrak{h}$ that the property $\left(\mathrm{gH}_{\text {o.s. }}\right)$ is inherited. Since $\mathfrak{h}$ satisfies $\left(\mathrm{gH}_{\alpha}\right)$, we can write

$$
H_{0}(x)=h_{0}(x) \cdot \xi_{\alpha} \xi_{\alpha}^{T}, \quad x \in\left(\sigma_{0}, \sigma_{1}\right),
$$

with some scalar function $h_{0}$. It follows that

$$
\circlearrowleft_{\beta} H_{0}(x)=h_{0}(x) \cdot\left(N_{\beta} \xi_{\alpha}\right)\left(N_{\beta} \xi_{\alpha}\right)^{T}=h_{0}(x) \cdot \xi_{\alpha-\beta} \xi_{\alpha-\beta}^{T},
$$

and hence $\circlearrowleft_{\beta} \mathfrak{h}$ satisfies $\left(\mathrm{gH}_{\alpha-\beta}\right)$.

2.27 Lemma. Let $\beta \in \mathbb{R}$. Then $q \in \mathcal{N}_{<\infty}^{(\infty)}$ if and only if $\circlearrowleft_{\beta} q \in \mathcal{N}_{<\infty}^{(-\cot \beta)}$. 
Proof. The case when $\beta \in \pi \mathbb{Z}$ is trivial. So let us assume that $\beta \notin \pi \mathbb{Z}$. We have

$$
\left(\circlearrowleft_{\beta} q\right)(z)=\frac{\cos \beta \cdot q(z)+\sin \beta}{-\sin \beta \cdot q(z)+\cos \beta}=\frac{\cot \beta \cdot q(z)+1}{-q(z)+\cot \beta}
$$

and therefore

$$
\begin{aligned}
\frac{1}{-\cot \beta-\left(\circlearrowleft_{\beta} q\right)(z)} & =\frac{-q+\cot \beta}{q \cot \beta-\cot ^{2} \beta-(q \cot \beta+1)} \\
& =\frac{1}{1+\cot ^{2} \beta} \cdot(q(z)-\cot \beta) .
\end{aligned}
$$

Hence

$$
\begin{aligned}
\circlearrowleft_{\beta} q \in \mathcal{N}_{<\infty}^{(-\cot \beta)} & \Longleftrightarrow \frac{1}{-\cot \beta-\circlearrowleft_{\beta} q} \in \mathcal{N}_{<\infty}^{(\infty)} \quad \text { (by definition) } \\
& \Longleftrightarrow \frac{1}{1+\cot ^{2} \beta} \cdot(q-\cot \beta) \in \mathcal{N}_{<\infty}^{(\infty)} \\
& \Longleftrightarrow q \in \mathcal{N}_{<\infty}^{(\infty)} .
\end{aligned}
$$

\section{Characterisation of Titchmarsh-Weyl coeffi- cients}

In this section we identify the properties $\left(\mathrm{gH}_{\text {o.s. }}\right),\left(\mathrm{gH}_{\alpha}\right)$ (defined in Definition 1.1 in the introduction) as being equivalent to the fact that the TitchmarshWeyl coefficient belongs to a class $\mathcal{N}_{<\infty}^{(\tau)}$ where $\tau=\cot \alpha$. This result is a typical instance of the intuition that the behaviour of the Hamiltonian at its left endpoint relates to the behaviour of the Titchmarsh-Weyl coefficient at $\infty$.

3.1 Theorem. Let $\mathfrak{h}$ be a singular general Hamiltonian with ind $\mathfrak{h}>0$ and let $\alpha \in[0, \pi)$. Then $\mathfrak{h} \in \mathfrak{H}_{\alpha}$ if and only if its Titchmarsh-Weyl coefficient $q_{\mathfrak{h}}$ belongs to the class $\mathcal{N}_{<\infty}^{(\cot \alpha)}$.

We first prove the statement for $\alpha=0$, in which case the class under consideration is $\mathcal{N}_{<\infty}^{(\infty)}$. The required statement for other values of $\alpha$ then follows easily by applying rotation isomorphisms. The method used for the proof is purely operator-theoretic: the desired equivalence follows, with the help of some $Q$ function theory, from the computation of the algebraic eigenspace of a certain operator $A$ at $\infty$, which is discussed in Lemma 3.2 below. For this we recall some concepts and notations. Let $S(\mathfrak{h}):=\operatorname{ker}\left(\pi_{l} \circ \Gamma(\mathfrak{h})\right)$ be the closed symmetric operator whose adjoint is $T(\mathfrak{h})$, where $T(\mathfrak{h})$ is the maximal linear relation in the boundary triple $(\mathcal{P}(\mathfrak{h}), T(\mathfrak{h}), \Gamma(\mathfrak{h}))$ associated with $\mathfrak{h}$, and let $A$ be the selfadjoint extension of $S(\mathfrak{h})$ defined by $A:=\operatorname{ker}\left(\pi_{l, 1} \circ \Gamma(\mathfrak{h})\right)$. In other words, the self-adjoint relation $A$ is obtained from $T(\mathfrak{h})$ by imposing a boundary condition at the regular left endpoint $\sigma_{0}$ (formally $f_{1}\left(\sigma_{0}\right)=0$ ); no boundary condition is needed at the singular endpoint $\sigma_{2}$. 
A given indefinite Hamiltonian can be decomposed into a finite number of 'elementary indefinite Hamiltonians' and positive definite Hamiltonians using the splitting points in the set $E$, where elementary indefinite Hamiltonians contain exactly one singularity; see $[\mathrm{KW} / \mathrm{IV}, \S 4]$. Unless $\operatorname{dim} \mathcal{P}\left(\mathfrak{h}_{i}\right)=1$, the model Pontryagin space corresponding to such an elementary indefinite Hamiltonian $\mathfrak{h}_{i}$ contains elements $\delta_{0}, \ldots, \delta_{\Delta_{i}-1+\ddot{o}_{i}}$ such that the pairs $\left(\delta_{k-1} ; \delta_{k}\right)$, $k=1, \ldots, \Delta_{i}-1+\ddot{o}_{i}$, are contained in the maximal relation $T\left(\mathfrak{h}_{i}\right)$; see $[\mathrm{KW} / \mathrm{IV}$, Definitions 4.5 and 4.11 and Proposition 4.17 (iv)]. If at least on one side of the singularity $H$ is just one indivisible interval of infinite length, then also $\left(0 ; \delta_{0}\right) \in T\left(\mathfrak{h}_{i}\right)$ by $[\mathrm{KW} / \mathrm{IV},(4.14)$ in Definition 4.11].

In the following lemma a connection between the algebraic eigenspace of $A$ at infinity and the behaviour of $H$ at $\sigma_{0}$ is established. In particular, this algebraic eigenspace at infinity depends on the fact whether $\mathfrak{h}$ starts with an indivisible interval of type 0 at $\sigma_{0}$ and the length of this indivisible interval.

3.2 Lemma. Let $\mathfrak{h}$ be a singular general Hamiltonian with $\kappa:=$ ind $_{-} \mathfrak{h}>0$, and let $\mathfrak{h}$ be given by the data $n, \sigma_{0}, \ldots, \sigma_{n+1}, H_{0}, \ldots, H_{n}, \ddot{o}_{i}, b_{i, 1}, \ldots, b_{i, \ddot{o}_{i}+1}$, $d_{i, 0}, \ldots, d_{i, 2 \Delta_{i}-1}$. Let $A$ be as above and denote by $\mathcal{E}_{A}(\infty)$ the algebraic eigenspace of $A$ at infinity.

(a) If $\mathfrak{h}$ does not start with an indivisible interval of type 0 , then $\mathcal{E}_{A}(\infty)=\{0\}$, i.e. $A$ is an operator.

(b) Assume that $\mathfrak{h}$ starts with a maximal indivisible interval of type 0 of finite positive length and let $s_{0}$ be the right endpoint of this interval. Moreover, decompose $\mathcal{P}(\mathfrak{h})$ as $\mathcal{P}(\mathfrak{h})=\mathcal{P}\left(\mathfrak{h}_{\varsigma_{s}}\right)[\dot{+}] \mathcal{P}\left(\mathfrak{h}_{s_{0} \longmapsto}\right)$. Then $\mathcal{E}_{A}(\infty)=\mathcal{P}\left(\mathfrak{h}_{\uparrow_{s}}\right)$.

(c) Assume that $\mathfrak{h}$ starts with an indivisible interval of type 0 of infinite length and that $\sigma_{1}$ is left endpoint of a maximal indivisible interval (which also must be of type 0) and let $s_{0}$ be the right endpoint of this interval. Moreover, decompose $\mathcal{P}(\mathfrak{h})$ as $\mathcal{P}(\mathfrak{h})=\mathcal{P}\left(\mathfrak{h}_{\uparrow s_{0}}\right)[\dot{+}] \mathcal{P}\left(\mathfrak{h}_{s_{0} \risingdotseq}\right)$. Then $\mathcal{E}_{A}(\infty)=\mathcal{P}\left(\mathfrak{h} \uparrow_{s_{0}}\right)$.

(d) Assume that $\mathfrak{h}$ starts with an indivisible interval of type 0 with infinite length and that $\sigma_{1}$ is not left endpoint of an indivisible interval. Choose a point $s_{0} \in\left(\sigma_{1}, \sigma_{2}\right)$ that is not inner point of an indivisible interval, and decompose $\mathcal{P}(\mathfrak{h})$ as $\mathcal{P}(\mathfrak{h})=\mathcal{P}\left(\mathfrak{h}_{\uparrow s_{0}}\right)[\dot{+}] \mathcal{P}\left(\mathfrak{h}_{s_{0} \risingdotseq}\right)$. Then

$$
\mathcal{E}_{A}(\infty)=\operatorname{span}\left\{\delta_{0}, \ldots, \delta_{\Delta_{1}+\ddot{o}_{1}-1}\right\} \subseteq \mathcal{P}\left(\mathfrak{h}_{\uparrow s_{0}}\right) .
$$

Moreover, in the cases $(b),(c)$ and $(d)$ the algebraic eigenspace $\mathcal{E}_{A}(\infty)$ is spanned by one maximal Jordan chain of $A$ at infinity and the dimension of a maximal $A^{-1}$-invariant non-positive subspace is equal to ind $\mathfrak{h}_{-} s_{0}$.

Proof. Let us first prove the statements in $(a)-(d)$. Assume that mul $A \neq\{0\}$, i.e. that there exists an element $(0 ; g) \in T(\mathfrak{h}), g \neq 0$, with $\pi_{l, 1} \Gamma(\mathfrak{h})(0 ; g)=0$. Since $S(\mathfrak{h})$ is an operator, we must have $\pi_{l, 2} \Gamma(\mathfrak{h})(0 ; g) \neq 0$. Hence $[\mathrm{KW} / \mathrm{V}$, Lemma 3.37] implies that $\mathfrak{h}$ starts with an indivisible interval of type 0 . This shows that the implication in $(a)$ is true.

Next assume that $\mathfrak{h}$ is of the form as described in $(b)$ or $(c)$ and let $s_{0}$ be the point specified there. Set

$$
A\left(\mathfrak{h}_{s_{0} \longmapsto}\right):=\operatorname{ker}\left(\pi_{l, 1} \circ \Gamma\left(\mathfrak{h}_{s_{0} \longmapsto}\right)\right), \quad S_{1}\left(\mathfrak{h}_{\urcorner}\right):=\operatorname{ker}\left(\left(\pi_{l, 1} \times \pi_{r}\right) \circ \Gamma\left(\mathfrak{h}_{\urcorner}\right)\right),
$$


and let $P_{\uparrow_{s}}$ and $P_{s_{0} \text { ґ }}$ be the orthogonal projections according to the decomposition $\mathcal{P}(\mathfrak{h})=\mathcal{P}\left(\mathfrak{h}_{\uparrow_{s_{0}}}\right)[\dot{+}] \mathcal{P}\left(\mathfrak{h}_{s_{0} ґ}\right)$.

We shall show that

$$
\mathcal{E}_{A}(\infty)=\bigcup_{n \in \mathbb{N}} \operatorname{mul} S_{1}\left(\mathfrak{h}_{\text {} s_{0}}\right)^{n} .
$$

First note that $S_{1}\left(\mathfrak{h}_{\uparrow s_{0}}\right) \subseteq A$, and hence the inclusion ' $\supseteq$ ' holds trivially since $\mathcal{E}_{A}(\infty)=\bigcup_{n \in \mathbb{N}}$ mul $A^{n}$. Conversely, let $g_{0}:=0$ and $g_{1}, \ldots, g_{n} \in \mathcal{P}(\mathfrak{h})$ be given such that

$$
\left(g_{l-1} ; g_{l}\right) \in A, \quad l=1, \ldots, n .
$$

Then, trivially, $\left(P_{\urcorner_{s_{0}}} g_{l-1} ; P_{\uparrow_{s_{0}}} g_{l}\right) \in S_{1}\left(\mathfrak{h}_{\urcorner_{s_{0}}}\right)^{*}$ and $\left(P_{s_{0} \risingdotseq} g_{l-1} ; P_{s_{0} \gg} g_{l}\right) \in T\left(\mathfrak{h}_{s_{0} \risingdotseq}\right)$. By the construction of the boundary relation $\Gamma\left(\mathfrak{h}_{\iota_{s}}\right)$, we have $\pi_{l, 1} \circ \Gamma\left(\mathfrak{h}_{\uparrow_{s}}\right)=$ $\pi_{r, 1} \circ \Gamma\left(\mathfrak{h}_{{ }^{\prime} s_{0}}\right)$; see $[\mathrm{KW} / \mathrm{IV}$, Definition 4.5]. Hence

$$
\pi_{l, 1} \circ \Gamma\left(\mathfrak{h}_{s_{0} \risingdotseq}\right)\left(P_{s_{0} \risingdotseq} g_{l-1} ; P_{s_{0} \risingdotseq} g_{l}\right)=\pi_{r, 1} \circ \Gamma\left(\mathfrak{h}_{\uparrow s_{0}}\right)\left(P_{\uparrow s_{0}} g_{l-1} ; P_{\uparrow s_{0}} g_{l}\right)=0
$$

for $l=1, \ldots, n$, and therefore $\left(P_{s_{0} \risingdotseq} g_{l-1} ; P_{s_{0} \risingdotseq} g_{l}\right) \in A\left(\mathfrak{h}_{s_{0} \risingdotseq}\right)$. Since $\mathfrak{h}_{s_{0} \gg}$ does not start with an indivisible interval of type 0 , this implies that $P_{s_{0}} g_{l}=0$, $l=1, \ldots, n$. If $\mathfrak{h}_{s_{0} \text { 队 }}$ is not just one indivisible interval of infinite length, it follows that

$$
\pi_{r} \circ \Gamma\left(\mathfrak{h}_{\urcorner_{s}}\right)\left(P_{\uparrow_{s} 0} g_{l-1} ; P_{\uparrow_{s}} g_{l}\right)=\pi_{l} \circ \Gamma\left(\mathfrak{h}_{s_{0} \risingdotseq}\right)\left(P_{s_{0} \risingdotseq} g_{l-1} ; P_{s_{0} \risingdotseq} g_{l}\right)=0,
$$

and hence that $\left(P_{\uparrow_{s} 0} g_{l-1} ; P_{\uparrow_{s_{0}}} g_{l}\right) \in S_{1}\left(\mathfrak{h}_{\uparrow s_{0}}\right)$. If $\mathfrak{h}_{s_{0} \longmapsto}$ is just one indivisible interval of type $\alpha$ of infinite length, then $\alpha \notin \pi \mathbb{Z}$ and

$$
\pi_{r} \circ \Gamma\left(\mathfrak{h}_{\uparrow s_{0}}\right)\left(P_{\uparrow_{s_{0}}} g_{l-1} ; P_{\uparrow s_{0}} g_{l}\right) \in \pi_{l} \circ \Gamma\left(\mathfrak{h}_{s_{0} \risingdotseq}\right)\left(P_{s_{0} \risingdotseq} g_{l-1} ; P_{s_{0} \risingdotseq} g_{l}\right)=\operatorname{span}\left\{J \xi_{\alpha}\right\} .
$$

However, $\pi_{r} \circ \Gamma\left(\mathfrak{h}_{\uparrow s_{0}}\right)\left(P_{\uparrow s_{0}} g_{l-1} ; P_{\uparrow s_{0}} g_{l}\right) \in \operatorname{span}\left\{\left(\begin{array}{l}0 \\ 1\end{array}\right)\right\}$ and $\alpha \notin \pi \mathbb{Z}$, and thus again $\left(P_{\uparrow s_{0}} g_{l-1} ; P_{\uparrow s_{0}} g_{l}\right) \in S_{1}\left(\mathfrak{h}_{\uparrow s_{0}}\right)$. This shows that the inclusion ' $\subseteq$ ' in (3.1) holds.

The Hamiltonian $\mathfrak{h}_{\uparrow s_{0}}$ is either positive definite and consists of just one indivisible interval (namely in case $(b)$ ), or (namely in case $(c)$ ) its rotation $\left.\circlearrowleft)_{\frac{\pi}{2}} \mathfrak{h}\right\urcorner_{s_{0}}$ is an elementary indefinite Hamiltonian of kind (B) or (C); see [KW/IV, $\S 4]$. In each of these cases, inspection of the definition of $\Gamma\left(\mathfrak{h} \wedge s_{0}\right)$ shows that

$$
\bigcup_{n \in \mathbb{N}} \operatorname{mul} S_{1}\left(\mathfrak{h}_{\uparrow s_{0}}\right)^{n}=\mathcal{P}\left(\mathfrak{h}_{\uparrow s_{0}}\right) .
$$

Together with (3.1) this shows the assertions in $(b)$ and $(c)$.

To prove $(d)$, assume that $\left(\sigma_{0}, \sigma_{1}\right)$ is indivisible of type 0 and $\sigma_{1}$ is not left endpoint of an indivisible interval. Our aim is to show that, independently of the choice of $s_{0}$, again the relation (3.1) holds. The inclusion ' $\supseteq$ ' is of course again trivial. For the converse inclusion, let $g_{0}:=0$ and $g_{1}, \ldots, g_{n} \in \mathcal{P}(\mathfrak{h})$ be given such that (3.2) holds. Choose $s_{1} \in\left(\sigma_{1}, s_{0}\right)$ such that $s_{1}$ is not left endpoint of an indivisible interval of type 0 and that $\operatorname{dim} \mathfrak{K}\left(\omega_{\mathfrak{h}}\left(s_{1}, \cdot\right)^{-1} \omega_{\mathfrak{h}}\left(s_{0}, \cdot\right)\right)>n$, where the reproducing kernel space $\mathfrak{K}(W)$ for a matrix function $W$ is defined in $\S 2$.b. The choice of $s_{1}$ is possible because $\left(\sigma_{1}, \sigma_{2}\right)$ does not start with an indivisible interval at $\sigma_{1}$. Next, choose $s_{2} \in\left(\sigma_{1}, s_{1}\right)$ such that $\operatorname{dim} \mathfrak{K}\left(\omega_{\mathfrak{h}}\left(s_{2}, \cdot\right)^{-1} \omega_{\mathfrak{h}}\left(s_{1}, \cdot\right)\right)>n$. Since

$$
\left(\left.\left(\psi(\mathfrak{h}) g_{l-1}\right)\right|_{\left(s_{2}, s_{0}\right)} ;\left.\left(\psi(\mathfrak{h}) g_{l}\right)\right|_{\left(s_{2}, s_{0}\right)}\right) \in T_{\max }\left(\left.H_{1}\right|_{\left(s_{2}, s_{0}\right)}\right),
$$


we obtain from $\left[\mathrm{KW} / \mathrm{IV}\right.$, Lemma 2.23] that $g_{l-1}\left(s_{1}\right)=0, l=1, \ldots, n$. This gives $\left(P_{s_{1} \gg} g_{l-1} ; P_{s_{1} ґ} g_{l}\right) \in S\left(\mathfrak{h}_{s_{1} \gg}\right)$. However, $S\left(\mathfrak{h}_{s_{1} \gg}\right)$ is an operator, and hence $P_{s_{1} \triangleright} g_{l}=0, l=1, \ldots, n$. We see that $\left(g_{l-1} ; g_{l}\right) \in S_{1}\left(\mathfrak{h}_{\uparrow s_{1}}\right) \subseteq S_{1}\left(\mathfrak{h}_{\uparrow s_{0}}\right)$, and hence that (3.1) holds.

In order to compute $\bigcup_{n \in \mathbb{N}} \operatorname{mul} S_{1}\left(\mathfrak{h}_{\uparrow s_{0}}\right)^{n}$, note first that

$$
\delta_{0}, \ldots, \delta_{\Delta_{1}+\ddot{o}_{1}-1} \in \bigcup_{n \in \mathbb{N}} \operatorname{mul} S_{1}\left(\mathfrak{h}_{{ } s_{0}}\right)^{n}
$$

by the properties of the $\delta_{k}$; see the paragraphs before this lemma. Hence it suffices to show that the dimension of $\bigcup_{n \in \mathbb{N}}$ mul $S_{1}\left(\mathfrak{h}_{\uparrow s_{0}}\right)^{n}$ cannot exceed $\Delta_{1}+\ddot{o}_{1}$. Let again $\left(g_{l-1} ; g_{l}\right) \in S_{1}\left(\mathfrak{h}_{\mathfrak{s} s_{0}}\right), l=1, \ldots, n, g_{0}=0$, be given. For $z \in \mathbb{C}$ denote

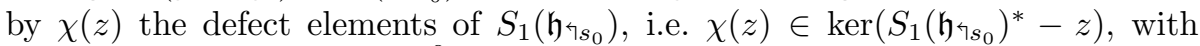
$\pi_{l} \circ \Gamma\left(\mathfrak{h}_{\uparrow_{s}}\right)(\chi(z) ; z \chi(z))=\left(\begin{array}{l}0 \\ 1\end{array}\right)$. Then

$$
\left[g_{l}, \chi(z)\right]=\bar{z}\left[g_{l-1}, \chi(z)\right], \quad l=1, \ldots, n,
$$

and hence, by induction, $g_{l} \perp \operatorname{span}\{\chi(z): z \in \mathbb{C}\}, l=1, \ldots, n$. Applying the isomorphism $\Xi_{s_{0}}$, as defined at the end of $\S 2$.e, from $\mathcal{P}\left(\mathfrak{h}_{\uparrow_{s_{0}}}\right)$ onto $\mathfrak{K}\left(\omega_{\mathfrak{h}}\left(s_{0}\right)\right)$, we find that $\Xi_{s_{0}} g_{l} \in \operatorname{ker} \pi_{-}$, where $\pi_{-}$is the projection onto the second component. However, we know from [Wo, Lemma 6.3 (proof, subcase 3b)] that dim ker $\pi_{-}=$ $\Delta_{1}+\ddot{o}_{1}$. As we noted above, this estimate suffices to complete the proof of statement $(d)$.

Let us now prove the last statements. If $\operatorname{dim} \mathcal{E}_{A}(\infty)=1$ in $(b)$ or $(c)$, these are trivial. Otherwise, in case $(b)$ or $(c)$, the algebraic eigenspace $\mathcal{E}_{A}(\infty)$ is spanned by the Jordan chain

$$
\delta_{0}, \ldots, \delta_{\ddot{o}_{1}}, \frac{1}{b_{1,1}}\left(p_{0}-\sum_{l=2}^{\ddot{o}_{1}+1} b_{1, l} \delta_{2+\ddot{o}_{1}-l}\right) ;
$$

see [KW/IV, Definition 4.5]. In case $(d), \mathcal{E}_{A}(\infty)$ is spanned by the Jordan chain $\delta_{0}, \ldots, \delta_{\Delta_{1}+\ddot{o}_{1}-1}$ as we have seen above. In both cases the Gram matrix of $\mathcal{E}_{A}(\infty)$ with respect to one of these Jordan chains has Hankel form, namely, (with $c_{1, j}$ as in (2.19))

$$
\left(\begin{array}{ccc}
0 & \cdots & c_{1,1} \\
\vdots & . \cdot & \vdots \\
c_{1,1} & \cdots & *
\end{array}\right) \quad \text { or } \quad\left(\begin{array}{ccc|ccc}
0 & \cdots & 0 & 0 & \cdots & 0 \\
\vdots & & \vdots & \vdots & & \vdots \\
0 & \cdots & 0 & 0 & \cdots & 0 \\
\hline 0 & \cdots & 0 & 0 & \cdots & c_{1,1} \\
\vdots & & \vdots & \vdots & . \cdot & \vdots \\
0 & \cdots & 0 & c_{1,1} & \cdots & c_{1, o_{1}}
\end{array}\right)
$$

in cases (b) and (c) and case (d), respectively, where the first matrix is of size $\ddot{o}_{1}+2$ and second matrix of size $\Delta_{1}+\ddot{o}_{1}$. The dimension of a maximal $A^{-1}$ invariant non-positive subspace is equal to the size of the maximal negative semidefinite square sub-matrix of the Gram matrix located at the top left corner. Such a maximal sub-matrix is either the zero matrix or a matrix that has zeros apart from one negative entry at the bottom right corner. Since such an entry at the bottom right corner is $c_{1,1}$, in cases (b) and (c) the maximal size of such 
a negative semi-definite sub-matrix is equal to

$$
\begin{aligned}
& \left\lfloor\frac{\ddot{o}_{1}+2}{2}\right\rfloor+\left\{\begin{array}{ll}
1 & \text { if } \ddot{o}_{1}+2 \text { is odd and } c_{1,1}<0 \\
0 & \text { otherwise }
\end{array}\right\} \\
& =\left\lfloor\frac{\ddot{o}_{1}}{2}\right\rfloor+1+\left\{\begin{array}{ll}
1 & \text { if } \ddot{o}_{1} \text { is odd and } b_{1,1}>0 \\
0 & \text { otherwise }
\end{array}\right\}=\text { ind }-\mathfrak{h}_{\urcorner_{0}}
\end{aligned}
$$

according to (2.13) since $\Delta_{1}=1$ in this case. Similarly, for the case (d) the dimension of a maximal $A^{-1}$-invariant non-positive subspace is equal to

$$
\Delta_{1}+\left\lfloor\frac{\ddot{o}_{1}}{2}\right\rfloor+\left\{\begin{array}{ll}
1 & \text { if } \ddot{o}_{1} \text { is odd and } b_{1,1}>0 \\
0 & \text { otherwise }
\end{array}\right\}=\operatorname{ind}_{-} \mathfrak{h}_{\uparrow_{\text {ง }}},
$$

which finishes the proof.

Proof (of Theorem 3.1; Case $\alpha=0$ ). We use the same notation as in the above lemma. By $\left[\mathrm{KW} / \mathrm{V}\right.$, Proposition 5.19] the Titchmarsh-Weyl coefficient $q_{\mathfrak{h}}$ of $\mathfrak{h}$ is a $\mathrm{Q}$-function of $S(\mathfrak{h})$ generated by $A$. Moreover, $S(\mathfrak{h})$ is completely nonself-adjoint by $\left[\mathrm{KW} / \mathrm{IV}\right.$, Theorem 8.6], and hence $A$ is minimal. Thus $q_{\mathfrak{h}}$ has a minimal representation of the form (2.4) in terms of $A$ and hence we can apply Lemma 2.5 , i.e. the asymptotics of $q_{\mathfrak{h}}$ at infinity reflects precisely the geometric structure of $\mathcal{E}_{A}(\infty)$.

First assume that $q_{\mathfrak{h}} \in \mathcal{N}_{\kappa}^{(\infty)}$. If $\mathfrak{h}$ does not start with an indivisible interval of type 0 , then $\lim _{z \rightarrow i \infty} \frac{1}{z} q_{\mathfrak{h}}(z)=0$ by [KW/V, Proposition 6.1$]$, which is a contradiction to the assumption that $q \in \mathcal{N}_{\kappa}^{(\infty)}$. If $\mathfrak{h}$ starts with an indivisible interval of type 0 and finite (positive) length $l$, then $\lim _{z \rightarrow i \infty} \frac{1}{z} q_{\mathfrak{h}}(z)=l \in$ $(0, \infty)$, which is again a contradiction. Hence $\mathfrak{h}$ starts with an indivisible interval of type 0 of infinite length, i.e. $\left(\mathrm{gH}_{0}\right)$ is satisfied. By Lemmas 2.5 and 3.2, we have ind $(\mathfrak{h})=\kappa=\nu_{\infty}(A)=\operatorname{ind}_{-}\left(\mathfrak{h}_{\uparrow_{s}}\right)$, where $s_{0}$ is as in Lemma 3.2. This implies that $\sigma_{1}$ is the only singularity of $\mathfrak{h}$ (since each singularity contributes at least one negative square), i.e. $\left(\mathrm{gH}_{\text {o.s. }}\right)$ is satisfied.

Now assume that $\left(\mathrm{gH}_{0}\right)$ and $\left(\mathrm{gH}_{\mathrm{o.s.}}\right)$ are satisfied. It follows from $\left(\mathrm{gH}_{0}\right)$ that in Lemma 3.2 either case $(\mathrm{c})$ or $(\mathrm{d})$ occurs. Hence $\nu_{\infty}(A)=\operatorname{ind}_{-}\left(\mathfrak{h}_{\uparrow_{s_{0}}}\right)$. The assumption $\left(\mathrm{gH}_{\text {o.s. }}\right)$ implies that ind ${ }_{-}\left(\mathfrak{h}_{\uparrow_{s_{0}}}\right)=$ ind $_{-}(\mathfrak{h})=\kappa$. By Lemma 2.5 it follows that $q_{\mathfrak{h}} \in \mathcal{N}_{\kappa}^{(\infty)}$.

Proof (of Theorem 3.1; General values of $\alpha$ ). Let a singular general Hamiltonian with ind $\mathfrak{h}_{-}>0$ be given which satisfies $\left(\mathrm{gH}_{\text {o.s. }}\right)$, and let $\alpha \in(0, \pi)$.

By Lemma 2.26, $\mathfrak{h}$ satisfies $\left(\mathrm{gH}_{\alpha}\right)$ if and only if the general Hamiltonian $\circlearrowleft_{\alpha} \mathfrak{h}$ satisfies $\left(\mathrm{gH}_{0}\right)$. We have $q_{\circlearrowleft_{\alpha} \mathfrak{h}}=N_{\alpha} \star q_{\mathfrak{h}}$. By Lemma 2.27, $q_{\mathfrak{h}} \in \mathcal{N}_{<\infty}^{(\cot \alpha)}$ if and only if $q_{\circlearrowleft_{\alpha} \mathfrak{h}} \in \mathcal{N}_{<\infty}^{(\infty)}$.

Using the already proved case ' $\alpha=0$ ' of Theorem 3.1 we conclude that $\mathfrak{h}$ satisfies $\left(\mathrm{gH}_{\alpha}\right)$ if and only if $q_{\mathfrak{h}} \in \mathcal{N}_{<\infty}^{(\cot \alpha)}$.

\section{Partial continuity of the fundamental solution}

Let $\alpha \in[0, \pi)$ and $\mathfrak{h} \in \mathfrak{H}_{\alpha}$. Our aim in this section is to show that, after an appropriate rotation, three entries of the (finite) maximal chain of matrices associated with $\mathfrak{h}$ are continuous at the singularity of $\mathfrak{h}$. 
4.1 Theorem. Let $\alpha \in[0, \pi)$ and $\mathfrak{h} \in \mathfrak{H}_{\alpha}$. Moreover, let $\omega_{\mathfrak{h}}$ be the (finite) maximal chain of matrices associated with $\mathfrak{h}$, and set $\mathfrak{t}_{\mathfrak{h}}(x):=\mathfrak{t}\left(\omega_{\mathfrak{h}}(x ; \cdot)\right)$, where $\mathfrak{t}$ is defined in (2.5). Then the following limit relations hold locally uniformly on $\mathbb{C}$ :

$\left(\lim _{\alpha}\right)$

$$
\begin{aligned}
& \lim _{x \searrow \sigma_{1}} \xi_{\alpha-\frac{\pi}{2}}^{T} \omega_{\mathfrak{h}}(x ; z) \xi_{\alpha-\frac{\pi}{2}}=\lim _{x \searrow \sigma_{1}} \xi_{\alpha}^{T} \omega_{\mathfrak{h}}(x ; z) \xi_{\alpha}=1, \\
& \lim _{x \searrow \sigma_{1}} \xi_{\alpha-\frac{\pi}{2}}^{T} \omega_{\mathfrak{h}}(x ; z) \xi_{\alpha}=0
\end{aligned}
$$

$\left(\lim _{\alpha}^{\prime}\right) \quad \lim _{x \searrow \sigma_{1}} \frac{\xi_{\alpha}^{T} \omega_{\mathfrak{h}}(x ; z) \xi_{\alpha-\frac{\pi}{2}}}{\left|\mathfrak{t}_{\mathfrak{h}}(x)\right| \cdot z}=1$.

4.2 Remark.

( $i$ ) Note that the quotient on the left-hand side of $\left(\lim _{\alpha}^{\prime}\right)$ is an entire function in $z$ since the numerator is zero for $z=0$. Expressions like this have to be interpreted as derivatives in the following.

(ii) The limit relations in $\left(\lim _{\alpha}\right)$ and $\left(\lim _{\alpha}^{\prime}\right)$ hold trivially when $t$ approaches $\sigma_{1}$ from the left. This follows since

$$
\omega_{\mathfrak{h}}(x ; z)=N_{\alpha-\frac{\pi}{2}}^{*}\left(\begin{array}{cc}
1 & 0 \\
-\mathfrak{t}_{\mathfrak{h}}(x) z & 1
\end{array}\right) N_{\alpha-\frac{\pi}{2}},
$$

and hence, for $x \in\left[\sigma, \sigma_{1}\right)$,

$$
\begin{aligned}
& \xi_{\alpha-\frac{\pi}{2}}^{*} \omega_{\mathfrak{h}}(x ; z) \xi_{\alpha-\frac{\pi}{2}}=\xi_{\alpha}^{T} \omega_{\mathfrak{h}}(x ; z) \xi_{\alpha}=1, \quad \xi_{\alpha-\frac{\pi}{2}}^{*} \omega_{\mathfrak{h}}(x ; z) \xi_{\alpha}=0, \\
& \frac{\xi_{\alpha}^{T} \omega_{\mathfrak{h}}(x ; z) \xi_{\alpha-\frac{\pi}{2}}}{-\mathfrak{t}_{\mathfrak{h}}(x) \cdot z}=1 .
\end{aligned}
$$

Hence $\left(\lim _{\alpha}\right)$ indeed expresses continuity at $\sigma_{1}$.

(iii) By (2.9) we have

$$
\mathfrak{t}_{\mathfrak{h}}(x)=\mathfrak{t}_{\mathfrak{h}}\left(x_{0}\right)-\int_{x}^{x_{0}} \operatorname{tr} H(t) d t
$$

for any $x_{0} \in\left(\sigma_{1}, \sigma_{2}\right)$ and, since $H$ is in limit point case at $\sigma_{1}$, thus $\mathfrak{t}_{\mathfrak{h}}(x) \rightarrow$ $-\infty$ as $x \searrow \sigma_{1}$. Hence

$$
\lim _{t \rightarrow \sigma_{1}}\left|\xi_{\alpha}^{T} \omega_{\mathfrak{h}}(x ; z) \xi_{\alpha-\frac{\pi}{2}}\right|=\infty
$$

locally uniformly on $\mathbb{C} \backslash\{0\}$.

(iv) For $\alpha=\frac{\pi}{2}$ the relations $\left(\lim _{\alpha}\right)$ and $\left(\lim _{\alpha}^{\prime}\right)$ read as follows:

$$
\begin{array}{ll}
\left(\lim _{\frac{\pi}{2}}\right) & \lim _{x \searrow \sigma_{1}} \omega_{\mathfrak{h}}(x ; z)_{11}=\lim _{x \searrow \sigma_{1}} \omega_{\mathfrak{h}}(x ; z)_{22}=1, \quad \lim _{x \searrow \sigma_{1}} \omega_{\mathfrak{h}}(x ; z)_{12}=0 ; \\
\left(\lim _{\frac{\pi}{2}}^{\prime}\right) \quad \lim _{x \searrow \sigma_{1}} \frac{\omega_{\mathfrak{h}}(x ; z)_{21}}{\left|\mathfrak{t}_{\mathfrak{h}}(x)\right| \cdot z}=1 .
\end{array}
$$


The proof of Theorem 4.1 is quite elaborate. We divide the remainder of this section into several subsections according to the following plan:

a. We provide/recall some preliminary facts, among them a normal family argument for Pólya class functions.

b. We establish asymptotics of the fundamental solution of positive definite canonical systems of a particular kind.

c. We derive that $\left(\lim _{\frac{\pi}{2}}\right)$ already implies finer asymptotics of $\omega_{\mathfrak{h}}(x ; z)$.

d. We carry out an inductive process to establish Theorem 4.1 for $\alpha=\frac{\pi}{2}$.

e. We apply rotation isomorphisms to obtain the asserted limit relations for arbitrary values of $\alpha$.

f. We prove a continuity result for the fundamental solution of an arbitrary general Hamiltonian.

\section{a. Preliminary observations.}

In order to justify a later application of [dB, Theorem 41], we need the following elementary reformulation of $\left(\mathrm{I}_{0}^{-}\right)$and $\left(\mathrm{HS}_{0}^{-}\right)$.

4.3 Lemma. Let $h_{11}$ and $h_{22}$ be locally integrable non-negative functions on an interval $\left(a, x_{0}\right]$. Assume that $\int_{a}^{x_{0}} h_{11}(x) d x<\infty$, set $\alpha(x):=\int_{a}^{t} h_{11}(t) d t$, and let $\gamma(t)$ be an anti-derivative of $h_{22}$ that is absolutely continuous on each compact interval contained in $\left(a, x_{0}\right]$, e.g. $\gamma_{0}(x):=\int_{x_{0}}^{x} h_{22}(t) d t$. Then

$$
\int_{a}^{x_{0}} \alpha(x) d \gamma(x)<\infty \Longleftrightarrow \int_{a}^{x_{0}}|\gamma(x)| d \alpha(x)<\infty .
$$

In this case, $\lim _{x \searrow a} \alpha(x) \gamma(x)=0$.

Proof. The measure $d \alpha$ is finite on $\left(a, x_{0}\right]$, and the measure space $\left\langle\left(a, x_{0}\right], d \gamma\right\rangle$ (the $\sigma$-algebra is the Borel algebra) is $\sigma$-finite since $h_{22}$ is locally integrable. Moreover, the function $\chi_{\{y \leq x\}}(x, y) h_{11}(y) h_{22}(x)$ is measurable and nonnegative. Hence the application of Fubini's theorem is justified, and we obtain

$$
\begin{aligned}
& \int_{a}^{x_{0}} \alpha(x) d \gamma_{0}(x)=\int_{a}^{x_{0}}\left(\int_{a}^{x} h_{11}(y) d y\right) h_{22}(x) d x \\
& =\int_{a}^{x_{0}}\left(\int_{y}^{x_{0}} h_{22}(x) d x\right) h_{11}(y) d y=\int_{a}^{x_{0}}\left(-\gamma_{0}(y)\right) d \alpha(y) .
\end{aligned}
$$

Any anti-derivative $\gamma$ of $h_{22}$ as in the statement of the lemma differs from $\gamma_{0}$ only by an additive constant. The integral on the left side of (4.1) does not depend on the choice of $\gamma$. Since $\int_{a}^{x_{0}} d \alpha(x)<\infty$ and $\gamma$ is non-decreasing, also finiteness/infiniteness of the integral on the right-hand side of (4.1) does not depend on the choice of $\gamma$. 
Assume that the integrals in (4.1) are finite. Let $x \in\left(a, x_{0}\right)$. Since $\alpha$ and $\gamma$ are both locally absolutely continuous on $\left(a, x_{0}\right]$, we may integrate by parts to obtain

$$
\int_{x}^{x_{0}} \alpha(t) d \gamma_{0}(t)=-\alpha(x) \gamma_{0}(x)-\int_{x}^{x_{0}} \gamma_{0}(t) d \alpha(t), \quad x \in\left(a, x_{0}\right] .
$$

Passing to the limit $x \searrow a$ and remembering (4.2), we find that $\alpha(x) \gamma_{0}(x)$ tends to 0 . Again this property is inherited by any other anti-derivative $\gamma$.

We shall use a normal family argument, which appeared already in the proof of $[\mathrm{dB}$, Theorem 41]. Denote by $\mathbb{P}$ the set of all real entire functions $F$ that belong to the Pólya class. Moreover, for $c>0$, set

$$
\mathbb{P}_{c}:=\left\{F \in \mathbb{P}: F(0)=1,\left|F^{\prime}(0)\right| \leq c,\left|F^{\prime \prime}(0)\right| \leq c\right\}
$$

4.4 Lemma. For each $c>0$ the class $\mathbb{P}_{c}$ is a normal family, i.e. every sequence of functions from $\mathbb{P}_{c}$ contains a subsequence that converges uniformly on compact sets. Moreover,

$$
\bigcap_{c>0} \mathbb{P}_{c}=\{1\}
$$

Proof. By [dB, Problems 10 and 13] each function $F \in \mathbb{P}$ with $F(0)=1$ satisfies the estimate

$$
\log |F(z)| \leq F^{\prime}(0) \operatorname{Re} z+\frac{1}{2}\left(F^{\prime}(0)^{2}-F^{\prime \prime}(0)\right)|z|^{2}, \quad z \in \mathbb{C} .
$$

Hence each class $\mathbb{P}_{c}$ is locally uniformly bounded and by Montel's theorem normal.

Assume that $F \in \bigcap_{c>0} \mathbb{P}_{c}$; then $F^{\prime}(0)=F^{\prime \prime}(0)=0$. By the above estimate, $F$ is bounded by 1 throughout $\mathbb{C}$, and hence constant. However, $F(0)=1$, and thus $F$ must be identically equal to 1 .

4.5 Lemma. Let $\left(F_{n}\right)_{n \in \mathbb{N}}$ be a sequence of functions $F_{n} \in \mathbb{P}$ with $F_{n}(0)=1$, $n \in \mathbb{N}$. If, for some real number $\beta$, we have

$$
\lim _{n \rightarrow \infty} F_{n}^{\prime}(0)=\beta, \quad \lim _{n \rightarrow \infty} F_{n}^{\prime \prime}(0)=\beta^{2},
$$

then $\lim _{n \rightarrow \infty} F_{n}(z)=e^{\beta z}$ locally uniformly on $\mathbb{C}$.

Proof. Consider the functions $G_{n}(z):=F_{n}(z) e^{-\beta z} ;$ then

$$
G_{n}^{\prime}(0)=F_{n}^{\prime}(0)-\beta, \quad G_{n}^{\prime \prime}(0)=F_{n}^{\prime \prime}(0)-2 \beta F_{n}^{\prime}(0)+\beta^{2} .
$$

Hence $\lim _{n \rightarrow \infty} G_{n}^{\prime}(0)=\lim _{n \rightarrow \infty} G_{n}^{\prime \prime}(0)=0$.

For some appropriate $c>0$ we have $\left\{G_{n}: n \in \mathbb{N}\right\} \subseteq \mathbb{P}_{c}$, and hence the sequence $\left(G_{n}\right)_{n \in \mathbb{N}}$ has accumulation points with respect to locally uniform convergence. Let $G$ be any such accumulation point. Then $G^{\prime}(0)=G^{\prime \prime}(0)=0$, and hence $G \in \bigcap_{c>0} \mathbb{P}_{c}$ and, by Lemma $4.4, G$ is identically equal to 1 . Therefore we can conclude that $\lim _{n \rightarrow \infty} G_{n}=1$ locally uniformly. 


\section{b. Asymptotics for a class of positive definite Hamiltonians.}

In this subsection we consider the class of positive definite Hamiltonians $H$ satisfying the following two properties (let $(a, b)$ be the domain of $H$ ):

$\left(\mathbf{G}_{1}\right)$ the Hamiltonian $H$ satisfies $\left(\mathrm{I}_{0}^{-}\right)$and $\left(\mathrm{HS}_{0}^{-}\right)$, and the interval $(a, b)$ is not indivisible;

$\left(\mathbf{G}_{2}\right)$ for one (and hence for all) $x_{0} \in(a, b)$ the limit

$$
\lim _{s \searrow a} \int_{s}^{x_{0}} h_{12}(x) d x
$$

exists and is finite.

Note that, if $H$ is in the limit circle case at $a$ and $(a, b)$ is not indivisible, then these conditions are trivially satisfied.

For each $s \in(a, b)$ let $W_{s t}(z)$ be the unique solution of

$$
\frac{d}{d t} W_{s t}(z) J=z W_{s t}(z) H(t), \quad t \in(a, b), \quad W_{s s}(z)=I,
$$

and write

$$
W_{s t}(z)=:\left(\begin{array}{ll}
A_{s t}(z) & B_{s t}(z) \\
C_{s t}(z) & D_{s t}(z)
\end{array}\right)
$$

Set

$$
\alpha(x):=\int_{a}^{x} h_{11}(t) d t, \quad \beta(x):=\lim _{s \searrow^{\prime}} \int_{s}^{x} h_{12}(t) d t,
$$

and let $\gamma$ be some absolutely continuous anti-derivative of $h_{22}$.

4.6 Remark.

(i) The condition $\left(\mathrm{G}_{2}\right)$ implies that $\lim _{t \searrow a} \beta(t)=0$. To see this, let $\varepsilon>0$ be given. Choose $t_{0} \in(a, b)$, then there exists $\delta>0$ such that

$$
\left|\int_{s}^{t_{0}} h_{12}(x) d x-\beta\left(t_{0}\right)\right|<\varepsilon, \quad s \in\left(a, t_{0}\right], s-a<\delta .
$$

Let $t \in\left(a, t_{0}\right], t-a<\delta$ and $s \in(a, t]$, then

$$
\left|\int_{s}^{t} h_{12}(x) d x\right|=\left|\int_{s}^{t_{0}} h_{12}(x) d x-\int_{t}^{t_{0}} h_{12}(x) d x\right|<2 \varepsilon .
$$

Passing to the limit $s \searrow a$ gives $|\beta(t)| \leq 2 \varepsilon$.

(ii) The assumption $\left(\mathrm{HS}_{0}^{-}\right)$implies that

$$
\int_{a}^{x}|\gamma(t)| d \alpha(t)<\infty \quad \text { for all } x \in(a, b)
$$


and hence, by Lemma 4.3 ,

$$
\int_{a}^{x} \alpha(t) d \gamma(t)<\infty \quad \text { for all } x \in(a, b)
$$

and

$$
\lim _{x \searrow a} \alpha(x) \gamma(x)=0 .
$$

Denote by $a_{+}$the maximal number in $[a, b)$ such that $\left(a, a_{+}\right)$is an indivisible interval of type $\frac{\pi}{2}$. Then

$$
\alpha(x) \begin{cases}=0, & x \in\left(a, a_{+}\right], \\ >0, & x \in\left(a_{+}, b\right),\end{cases}
$$

and in particular, $\lim _{x \searrow a_{+}} \alpha(x)=0$. The matrix function

$$
m(x):=\left(\begin{array}{cc}
\alpha(x) & \beta(x) \\
\beta(x) & \gamma(x)
\end{array}\right)
$$

satisfies $m(t)-m(s)=\int_{s}^{t} H(x) d x$, and hence is non-decreasing and locally absolutely continuous on $(a, b)$.

4.7. Existence of $\stackrel{\AA}{t}_{t}$ and $\stackrel{\circ}{B}_{t}$. By the definition of $a_{+}$the Hamiltonian $H$ does not start with an indivisible interval of type $\frac{\pi}{2}$ at the left endpoint of the interval $\left(a_{+}, b\right)$. Hence all hypotheses of $[\mathrm{dB}$, Theorem 41] are satisfied (with the right half-line $(0, \infty)$ substituted by the interval $\left.\left(a_{+}, b\right)\right)$. An application of this theorem provides us with families $\AA_{t}$ and $\stackrel{\circ}{B}_{t}, t \in\left(a_{+}, b\right)$, of real entire functions which have the following properties:

(a) For each $t \in\left(a_{+}, b\right)$ the function $\AA_{t}-i \stackrel{\circ}{B}_{t}$ has no real zeros and belongs to the Hermite-Biehler class $\mathcal{H} B_{0}$ and the Pólya class. Moreover, $\AA_{t}(0)=1$ and $\stackrel{\circ}{B}_{t}(0)=0$.

(b) We have

$$
\frac{d}{d t}\left(\stackrel{\circ}{A}_{t}(z), \stackrel{\circ}{B}_{t}(z)\right) J=z\left(\stackrel{\circ}{A}_{t}(z), \stackrel{\circ}{B}_{t}(z)\right) H(t), \quad t \in\left(a_{+}, b\right) .
$$

(c) $\lim _{t \searrow a_{+}} \AA_{t}=1$ and $\lim _{t \searrow a_{+}} \stackrel{\circ}{B}_{t}=0$ locally uniformly on $\mathbb{C}$.

(d) $\stackrel{\circ}{A}_{t}=\lim _{s \searrow a_{+}} A_{s t}$ and $\stackrel{\circ}{B_{t}}=\lim _{s \searrow_{a_{+}}} B_{s t}$ locally uniformly on $\mathbb{C}$.

For $(c)$ remember Remark 4.6. Item $(d)$ follows from the construction in the proof of [dB, Theorem 41]. Moreover, the functions $\AA_{t}$ and $\stackrel{\circ}{B}_{t}$ are uniquely determined by the properties (a), (b) and (c).

4.8 Remark. If $H$ is in the limit circle case at $a_{+}$, then $\left(\AA_{t}(z), \stackrel{\circ}{B}_{t}(z)\right)$ is the unique solution of (4.5) satisfying the initial condition $\left(\AA_{a_{+}}(z),{\stackrel{\circ}{a_{+}}}_{a}(z)\right)=(1,0)$. 
The key steps in the present context are the next two lemmas where we determine the asymptotics of $\stackrel{\circ}{B}_{t}, C_{s t}$ and $D_{s t}$.

4.9 Lemma. Assume that $H$ satisfies $\left(\mathrm{G}_{1}\right),\left(\mathrm{G}_{2}\right)$, and let $a_{+} \in[a, b)$ be the maximal number such that $\left(a, a_{+}\right)$is indivisible of type $\frac{\pi}{2}$. Then

$$
\lim _{t \searrow a_{+}} \frac{\stackrel{\circ}{B}_{t}(z)}{z \alpha(t)}=1
$$

locally uniformly in $\mathbb{C}$.

Proof. Consider the functions $A_{s t}$ and $B_{s t}$ defined in (4.3). By 2.13 the function $B_{s t}$ belongs to the Pólya class, and by $(d)$ above therefore also $B_{t}$ belongs to the Pólya class. By [dB, Lemma 7.1] the same is true for $\frac{\stackrel{B}{t}_{t}(z)}{z}$.

In order to apply Lemma 4.5 we compute the derivatives of $A_{s t}$ and $B_{s t}$ with respect to $z$ at 0 . For $s, t \in(a, b), s<t$, the function $x \mapsto\left(A_{s x}, B_{s x}\right), x \in[s, t]$, satisfies the differential equation (1.1) with Hamiltonian $\left.H\right|_{(s, t)}$, and takes the value $(1,0)$ for $x=s$. Hence

$$
\begin{aligned}
B_{s t}(z) & =z \int_{s}^{t} A_{s x}(z) d \alpha(x)+z \int_{s}^{t} B_{s x}(z) d \beta(x), \quad s<t<b, \\
1-A_{s t}(z) & =z \int_{s}^{t} A_{s x}(z) d \beta(x)+z \int_{s}^{t} B_{s x}(z) d \gamma(x), \quad s<t<b .
\end{aligned}
$$

Note that $\left.H\right|_{(s, t)}$ is in the limit circle case at both endpoints.

Dividing these equations by $z$, letting $z$ tend to 0 and observing that $W_{s t}(0)=I$, we obtain

$$
B_{s t}^{\prime}(0)=\alpha(t)-\alpha(s), \quad A_{s t}^{\prime}(0)=\beta(s)-\beta(t) .
$$

If we divide (4.8) by $z$, differentiate with respect to $z$ and evaluate at $z=0$, then we obtain

$$
\begin{aligned}
-\frac{A_{s t}^{\prime \prime}(0)}{2} & =\left(\frac{1-A_{s t}(z)}{z}\right)^{\prime}(0)=\int_{s}^{t} A_{s x}^{\prime}(0) d \beta(x)+\int_{s}^{t} B_{s x}^{\prime}(0) d \gamma(x) \\
& =\int_{s}^{t}[\beta(s)-\beta(x)] d \beta(x)+\int_{s}^{t}[\alpha(x)-\alpha(s)] d \gamma(x) \\
& =-\frac{1}{2}[\beta(s)-\beta(t)]^{2}+\int_{s}^{t} \alpha(x) d \gamma(x)-\alpha(s)[\gamma(t)-\gamma(s)] .
\end{aligned}
$$

Equation (4.7) gives

$$
\begin{aligned}
& \frac{B_{s t}^{\prime \prime}(0)}{2}=\left(\frac{B_{s t}(z)}{z}\right)^{\prime}(0)=\int_{s}^{t} A_{s x}^{\prime}(0) d \alpha(x)+\int_{s}^{t} B_{s x}^{\prime}(0) d \beta(x) \\
& =\int_{s}^{t}[\beta(s)-\beta(x)] d \alpha(x)+\int_{s}^{t}[\alpha(x)-\alpha(s)] d \beta(x)
\end{aligned}
$$




$$
\begin{aligned}
& =\beta(s)[\alpha(t)-\alpha(s)]-\int_{s}^{t} \beta(x) d \alpha(x)+\left.[\alpha(x)-\alpha(s)] \beta(x)\right|_{x=s} ^{t}-\int_{s}^{t} \beta(x) d \alpha(x) \\
& =[\beta(s)+\beta(t)] \cdot[\alpha(t)-\alpha(s)]-2 \int_{s}^{t} \beta(x) d \alpha(x) .
\end{aligned}
$$

It follows that

$$
\lim _{s \searrow a_{+}}\left(\frac{B_{s t}(z)}{z}\right)^{\prime}(0)=\beta(t) \alpha(t)-2 \int_{a_{+}}^{t} \beta(x) d \alpha(x),
$$

and, using the Mean Value Theorem to evaluate the integral,

$$
\lim _{t \searrow a_{+}}\left[\frac{1}{\alpha(t)} \lim _{s \searrow a_{+}}\left(\frac{B_{s t}(z)}{z}\right)^{\prime}(0)\right]=0 .
$$

Dividing (4.7) by $z$, differentiating twice and evaluating at $z=0$, we obtain

$$
\left(\frac{B_{s t}(z)}{z}\right)^{\prime \prime}(0)=\int_{s}^{t} A_{s x}^{\prime \prime}(0) d \alpha(x)+\int_{s}^{t} B_{s x}^{\prime \prime}(0) d \beta(x) .
$$

The first summand equals

$$
\begin{aligned}
& \int_{s}^{t} A_{s x}^{\prime \prime}(0) d \alpha(x) \\
& =\int_{s}^{t}\left([\beta(s)-\beta(x)]^{2}-2 \int_{s}^{x} \alpha(y) d \gamma(y)+2 \alpha(s)[\gamma(x)-\gamma(s)]\right) d \alpha(x) \\
& =\int_{s}^{t}[\beta(s)-\beta(x)]^{2} d \alpha(x)-2 \int_{s}^{t} \int_{s}^{x} \alpha(y) d \gamma(y) d \alpha(x)+2 \alpha(s) \int_{s}^{t}[\gamma(x)-\gamma(s)] d \alpha(x),
\end{aligned}
$$

the second one

$$
\begin{aligned}
& \int_{s}^{t} B_{s x}^{\prime \prime}(0) d \beta(x)=2 \int_{s}^{t}\left([\beta(s)+\beta(x)] \cdot[\alpha(x)-\alpha(s)]-2 \int_{s}^{x} \beta(y) d \alpha(y)\right) d \beta(x) \\
& =\int_{s}^{t}[\alpha(x)-\alpha(s)] \cdot 2[\beta(s)+\beta(x)] d \beta(x)-4 \int_{s}^{t} \int_{s}^{x} \beta(y) d \alpha(y) d \beta(x) \\
& =[\beta(s)+\beta(t)]^{2}[\alpha(t)-\alpha(s)]-\int_{s}^{t}[\beta(s)+\beta(x)]^{2} d \alpha(x) \\
& \quad-4 \int_{s}^{t}[\beta(t)-\beta(y)] \beta(y) d \alpha(y) .
\end{aligned}
$$


Using the Bounded Convergence Theorem, we conclude that (note here that $|\gamma(x)-\gamma(s)| \leq 2 \max \{|\gamma(t)|,|\gamma(s)|\}, x \in(s, t))$

$$
\begin{aligned}
\lim _{s \searrow a_{+}}\left(\frac{B_{s t}(z)}{z}\right)^{\prime \prime}(0)=\int_{a_{+}}^{t} \beta(x)^{2} d \alpha(x)-2 \int_{a_{+}}^{t} \int_{a_{+}}^{x} \alpha(y) d \gamma(y) d \alpha(x) \\
+\beta(t)^{2} \alpha(t)-\int_{a_{+}}^{t} \beta(x)^{2} d \alpha(x)-4 \int_{a_{+}}^{t}[\beta(t)-\beta(y)] \beta(y) d \alpha(y),
\end{aligned}
$$

and, again using the Mean Value Theorem, we obtain

$$
\lim _{t \searrow_{+}}\left[\frac{1}{\alpha(t)} \lim _{s a_{+}}\left(\frac{B_{s t}(z)}{z}\right)^{\prime \prime}(0)\right]=0 .
$$

Let

$$
F_{t}(z):=\frac{\stackrel{\circ}{B_{t}}(z)}{z \alpha(t)} .
$$

Since $\stackrel{\circ}{t}_{t}^{\prime}(0)=\lim _{s \searrow a_{+}} B_{s t}^{\prime}(0)=\alpha(t)$ and

$$
\left(\frac{\stackrel{\circ}{B}_{t}(z)}{z}\right)^{\prime}(0)=\lim _{s \searrow a_{+}}\left(\frac{B_{s t}(z)}{z}\right)^{\prime}(0), \quad\left(\frac{\stackrel{\circ}{B}_{t}(z)}{z}\right)^{\prime \prime}(0)=\lim _{s \searrow a_{+}}\left(\frac{B_{s t}(z)}{z}\right)^{\prime \prime}(0),
$$

it follows from (4.9) and (4.10) that

$$
F_{t}(0)=1, \quad \lim _{t \searrow a_{+}} F_{t}^{\prime}(0)=0, \quad \lim _{t \searrow a_{+}} F_{t}^{\prime \prime}(0)=0 .
$$

By Lemma 4.5 this implies that $\lim _{t \searrow a_{+}} F_{t}(z)=1$ locally uniformly on $\mathbb{C}$, which is $(4.6)$.

4.10 Lemma. Assume that $H$ satisfies $\left(\mathrm{G}_{1}\right),\left(\mathrm{G}_{2}\right)$.

(i) The limit

$$
\lim _{s \searrow a} D_{s t}^{\prime}(0)
$$

exists for every $t \in(a, b)$, and

$$
\lim _{t \searrow a} \lim _{s \searrow a} D_{s t}^{\prime}(0)=0 .
$$

(ii) If $\lim _{s \searrow a} \gamma(s) \neq 0$, then

$$
\lim _{s \searrow a} \frac{1}{\gamma(s)} D_{s t}^{\prime \prime}(0)
$$

exists for every $t \in(a, b)$ and

$$
\lim _{t \searrow a} \lim _{s \searrow a} \frac{1}{\gamma(s)} D_{s t}^{\prime \prime}(0)=0 .
$$


(iii) Let

$\tilde{I}:=\{t \in(a, b): t$ is not right endpoint of an indivisible interval

of type 0$\}$.

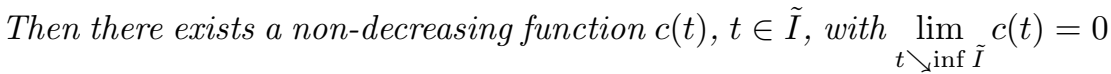
such that

$$
\frac{C_{s t}(z)}{(\gamma(s)-\gamma(t)) z} \in \mathbb{P}_{c(t)}, \quad a<s<t, t \in \tilde{I} .
$$

Proof. For $s, t \in(a, b), s<t$, the function $x \mapsto\left(C_{s x}, D_{s x}\right)$ satisfies the differential equation (1.1) with Hamiltonian $\left.H\right|_{(s, t)}$, and takes the value $(0,1)$ for $x=s$. Hence

$$
\begin{aligned}
D_{s t}(z)-1 & =z \int_{s}^{t} C_{s x}(z) d \alpha(x)+z \int_{s}^{t} D_{s x}(z) d \beta(x), \quad s<t<b, \\
-C_{s t}(z) & =z \int_{s}^{t} C_{s x}(z) d \beta(x)+z \int_{s}^{t} D_{s x}(z) d \gamma(x), \quad s<t<b .
\end{aligned}
$$

Note that $\left.H\right|_{(s, t)}$ is in the limit circle case at both endpoints. Dividing the first integral equation by $z$ and letting $z \rightarrow 0$ we easily see that

$$
D_{s t}^{\prime}(0)=\beta(t)-\beta(s), \quad C_{s t}^{\prime}(0)=\gamma(s)-\gamma(t),
$$

and hence that

$$
\lim _{s \searrow a} D_{s t}^{\prime}(0)=\beta(t), \quad \lim _{t \searrow a} \lim _{s \searrow a} D_{s t}^{\prime}(0)=0,
$$

which is $(i)$. Moreover,

$$
\begin{aligned}
\frac{D_{s t}^{\prime \prime}(0)}{2} & =\left(\frac{D_{s t}(z)-1}{z}\right)^{\prime}(0)=\int_{s}^{t} C_{s x}^{\prime}(0) d \alpha(x)+\int_{s}^{t} D_{s x}^{\prime}(0) d \beta(x) \\
& =\int_{s}^{t}[\gamma(s)-\gamma(x)] d \alpha(x)+\int_{s}^{t}[\beta(x)-\beta(s)] d \beta(x) \\
& =\gamma(s)[\alpha(t)-\alpha(s)]-\int_{s}^{t} \gamma(x) d \alpha(x)+\frac{1}{2}[\beta(t)-\beta(s)]^{2}
\end{aligned}
$$

Under the assumption that $\lim _{s \searrow^{a}} \gamma(s) \neq 0$, it follows that

$$
\lim _{s \searrow a} \frac{1}{\gamma(s)} D_{s t}^{\prime \prime}(0)=2 \alpha(t)-\lim _{y \searrow a}\left(\frac{1}{\gamma(y)}\right)\left[2 \int_{a}^{t} \gamma(x) d \alpha(x)+(\beta(t))^{2}\right] .
$$

Note that the integral exists by Remark $4.6(i i)$. If we now let $t \searrow a$, we obtain

$$
\lim _{t \searrow a s \searrow a} \frac{1}{\gamma(s)} D_{s t}^{\prime \prime}(0)=0 .
$$


Hence $(i i)$ is proved.

Finally, we show (iii). By (2.8) the function

$$
z \mapsto \frac{C_{s t}(z)}{(\gamma(s)-\gamma(t)) z}
$$

is in the Pólya class. To estimate $C_{s t}$, we compute

$$
\begin{aligned}
-\frac{C_{s t}^{\prime \prime}(0)}{2} & =-\left(\frac{C_{s t}(z)}{z}\right)^{\prime}(0)=\int_{s}^{t} C_{s x}^{\prime}(0) d \beta(x)+\int_{s}^{t} D_{s x}^{\prime}(0) d \gamma(x) \\
& =\int_{s}^{t}[\gamma(s)-\gamma(x)] d \beta(x)+\int_{s}^{t}[\beta(x)-\beta(s)] d \gamma(x) \\
& =[\gamma(s)-\gamma(t)] \cdot[\beta(t)-\beta(s)]+2 \int_{s}^{t}[\beta(x)-\beta(s)] d \gamma(x) .
\end{aligned}
$$

If $t \in \tilde{I}$, then $\gamma(t)>\gamma(s)$ and hence

$$
\begin{aligned}
& \left|\left(\frac{C_{s t}(z)}{(\gamma(s)-\gamma(t)) z}\right)^{\prime}(0)\right| \\
& \leq|\beta(t)-\beta(s)|+\frac{4}{\gamma(t)-\gamma(s)} \sup _{x \in(a, t]}|\beta(x)| \int_{s}^{t} d \gamma(x) \leq 6 \sup _{x \in(a, t]}|\beta(x)| .
\end{aligned}
$$

Next,

$$
-\left(\frac{C_{s t}(z)}{z}\right)^{\prime \prime}(0)=\int_{s}^{t} C_{s x}^{\prime \prime}(0) d \beta(x)+\int_{s}^{t} D_{s x}^{\prime \prime}(0) d \gamma(x) .
$$

The first summand can be written as

$$
\begin{aligned}
\int_{s}^{t} C_{s x}^{\prime \prime}(0) d \beta(x)=-2 \int_{s}^{t}[\gamma(s)-\gamma(x)] \cdot & {[\beta(x)-\beta(s)] d \beta(x) } \\
& -4 \int_{s}^{t} \int_{s}^{x}[\beta(y)-\beta(s)] d \gamma(y) d \beta(x) .
\end{aligned}
$$

The first integral on the right-hand side of this relation equals

$$
\begin{aligned}
& \int_{s}^{t}[\gamma(s)-\gamma(x)] \cdot[\beta(x)-\beta(s)] d \beta(x) \\
&=[\gamma(s)-\gamma(t)] \frac{[\beta(t)-\beta(s)]^{2}}{2}-\int_{s}^{t} \frac{[\beta(x)-\beta(s)]^{2}}{2} d \gamma(x),
\end{aligned}
$$


the second one

$$
\int_{s}^{t} \int_{s}^{x}[\beta(y)-\beta(s)] d \gamma(y) d \beta(x)=\int_{s}^{t}[\beta(t)-\beta(y)] \cdot[\beta(y)-\beta(s)] d \gamma(y) .
$$

Hence, for $t \in \tilde{I}$, we obtain the estimate

$$
\left|\frac{1}{\gamma(t)-\gamma(s)} \int_{s}^{t} C_{s x}^{\prime \prime}(0) d \beta(x)\right| \leq 8 \sup _{x \in(a, t]}|\beta(x)|^{2} .
$$

Next we compute

$$
\begin{aligned}
\int_{s}^{t} D_{s x}^{\prime \prime}(0) d \gamma(x)=2 \int_{s}^{t} \gamma(s) & {[\alpha(x)-\alpha(s)] d \gamma(x) } \\
& -2 \int_{s}^{t} \int_{s}^{x} \gamma(y) d \alpha(y) d \gamma(x)+\int_{s}^{t}[\beta(x)-\beta(s)]^{2} d \gamma(x) .
\end{aligned}
$$

The first integral on the right-hand side equals

$$
\begin{aligned}
& 2 \gamma(s) \int_{s}^{t} \alpha(x) d \gamma(x)-2 \gamma(s) \alpha(s) \int_{s}^{t} d \gamma(x) \\
& =2[\gamma(s)-\gamma(t)] \int_{s}^{t} \alpha(x) d \gamma(x)+2 \gamma(t) \int_{s}^{t} \alpha(x) d \gamma(x)-2 \gamma(s) \alpha(s)[\gamma(t)-\gamma(s)] .
\end{aligned}
$$

Hence, for $t \in \tilde{I}$, (we use that $\alpha$ is monotonic increasing)

$$
\begin{aligned}
& \left|\frac{1}{\gamma(s)-\gamma(t)} \int_{s}^{t} D_{s x}^{\prime \prime}(0) d \gamma(x)\right| \\
& \leq 2 \int_{s}^{t} \alpha(x) d \gamma(x)+\frac{2|\gamma(t)|}{\gamma(t)-\gamma(s)} \int_{s}^{t} \alpha(x) d \gamma(x)+2 \sup _{x \in(a, t]}|\gamma(x) \alpha(x)| \\
& \quad+\frac{2}{\gamma(t)-\gamma(s)} \int_{s}^{t} \int_{s}^{t}|\gamma(y)| d \alpha(y) d \gamma(x)+4 \sup _{x \in(a, t]}|\beta(x)|^{2} \\
& \leq 2 \int_{s}^{t} \alpha(x) d \gamma(x)+\frac{2|\gamma(t)|}{\gamma(t)-\gamma(s)} \int_{s}^{t} \alpha(t) d \gamma(x)+2 \sup _{x \in(a, t]}|\gamma(x) \alpha(x)| \\
& \quad+2 \int_{s}^{t}|\gamma(y)| d \alpha(y)+4 \sup _{x \in(a, t]}|\beta(x)|^{2}
\end{aligned}
$$




$$
\leq 2 \int_{a}^{t} \alpha(x) d \gamma(x)+4 \sup _{x \in(a, t]}|\gamma(x) \alpha(x)|+2 \int_{a}^{t}|\gamma(y)| d \alpha(y)+4 \sup _{x \in(a, t]}|\beta(x)|^{2} .
$$

Putting these estimates together we obtain

$$
\begin{aligned}
\left|\left(\frac{C_{s t}(z)}{(\gamma(s)-\gamma(t)) z}\right)^{\prime \prime}(0)\right| \leq & 2 \int_{a}^{t} \alpha(x) d \gamma(x)+4 \sup _{x \in(a, t]}|\gamma(x) \alpha(x)| \\
& +2 \int_{a}^{t}|\gamma(y)| d \alpha(y)+12 \sup _{x \in(a, t]}|\beta(x)|^{2} .
\end{aligned}
$$

It follows from (4.12), (4.13) and (4.14) that, for $t \in \tilde{I}$ and $s \in(a, t)$,

$$
\frac{C_{s t}(z)}{(\gamma(s)-\gamma(t)) z} \in \mathbb{P}_{c(t)}
$$

for a certain function $c(t)$, which can be chosen to be non-decreasing. Let $a^{\prime}:=\inf \tilde{I}$. If $a^{\prime}>a$, then $\left(a, a^{\prime}\right)$ is a maximal indivisible interval of type 0 . In this case $H$ must be in the limit circle case at $a$ by $\left(\mathrm{G}_{1}\right)$, and we have $\beta(t)=0$ and $\gamma(t)=0$ for $t \in\left[a, a^{\prime}\right]$, which implies that we can choose $c(t)$ such that $c(t) \rightarrow 0$ as $t \searrow a^{\prime}$. If $a^{\prime}=a$, then it follows from Remark 4.6 that we can choose $c(t)$ such that $c(t) \rightarrow 0$ as $t \searrow a$.

Note that the assertion (4.11) has a different meaning depending whether $H$ is in the limit point or limit circle case at $a$. In the limit point case it limits the growth of $C_{s t}$, whereas in the limit circle case it determines the speed of convergence. More precisely, for the latter case the following is true.

4.11 Corollary. Let $H$ be a Hamiltonian on $(a, b)$ which is in the limit circle case at $a$ and does not start with an indivisible interval of type 0 at a, and write

$$
\omega_{H}(t ; z)=:\left(\begin{array}{ll}
A_{t}(z) & B_{t}(z) \\
C_{t}(z) & D_{t}(z)
\end{array}\right)
$$

Then

$$
\lim _{t \searrow a} \frac{C_{t}(z)}{C_{t}^{\prime}(0) \cdot z}=1
$$

locally uniformly on $\mathbb{C}$.

Proof. We may pass to the limit $s \searrow a$ in (4.11), observe that $C_{t}^{\prime}(0)=\gamma(a)-\gamma(t)$ and apply Lemma 4.5 .

\section{c. Asymptotics for a class of general Hamiltonians.}

Let $\mathfrak{h} \in \mathfrak{H}_{\frac{\pi}{2}}$ and denote by $H$ the Hamiltonian function of $\mathfrak{h}$ on the interval $\left(\sigma_{1}, \sigma_{2}\right)$. Then $H$ is in limit point case at $\sigma_{1}$ and it satisfies $\left(\mathrm{G}_{1}\right)$ unless $\left(\sigma_{1}, \sigma_{2}\right)$ is indivisible (which cannot happen if $\mathfrak{h}$ is a singular general Hamiltonian). In order to shorten notation we write again

$$
\omega_{\mathfrak{h}}(t ; z)=:\left(\begin{array}{ll}
A_{t}(z) & B_{t}(z) \\
C_{t}(z) & D_{t}(z)
\end{array}\right)
$$


and

$$
\left.\frac{\partial}{\partial z} \omega_{\mathfrak{h}}(t ; z) J\right|_{z=0}=:\left(\begin{array}{cc}
\alpha(t) & \beta(t) \\
\beta(t) & \gamma(t)
\end{array}\right)
$$

Explicitly, this is

$$
\alpha(t)=B_{t}^{\prime}(0), \quad \beta(t)=-A_{t}^{\prime}(0)=D_{t}^{\prime}(0), \quad \gamma(t)=-C_{t}^{\prime}(0)
$$

and $\mathfrak{t}_{\mathfrak{h}}(t)=\alpha(t)+\gamma(t)$. It is easy to see that $\left.\frac{\partial}{\partial z} \omega_{\mathfrak{h}}(t ; z) J\right|_{z=0}$ is an anti-derivative of $H(t)$ and hence

$$
\alpha^{\prime}=h_{11}, \quad \beta^{\prime}=h_{12}, \quad \gamma^{\prime}=h_{22} .
$$

Moreover, if $A_{s t}, B_{s t}, C_{s t}, D_{s t}$ are as in (4.3), then

$$
\omega_{\mathfrak{h}}(s ; z)^{-1} \omega_{\mathfrak{h}}(t ; z)=\left(\begin{array}{ll}
A_{s t}(z) & B_{s t}(z) \\
C_{s t}(z) & D_{s t}(z)
\end{array}\right) .
$$

It follows that

$$
\left(\begin{array}{ll}
A_{s}^{\prime}(0) & B_{s}^{\prime}(0) \\
C_{s}^{\prime}(0) & D_{s}^{\prime}(0)
\end{array}\right)+\left(\begin{array}{ll}
A_{s t}^{\prime}(0) & B_{s t}^{\prime}(0) \\
C_{s t}^{\prime}(0) & D_{s t}^{\prime}(0)
\end{array}\right)=\left(\begin{array}{ll}
A_{t}^{\prime}(0) & B_{t}^{\prime}(0) \\
C_{t}^{\prime}(0) & D_{t}^{\prime}(0)
\end{array}\right) .
$$

\subsection{Remark.}

(i) It will turn out later that $H$ also satisfies $\left(\mathrm{G}_{2}\right)$, cf. Corollary 4.20 , but at the present stage this is not known.

(ii) If $H$ satisfies $\left(\mathrm{G}_{2}\right)$, then the functions $\alpha$ and $\beta$ defined by (4.16) may differ from the functions defined in (4.4) by additive constants.

(iii) Since $-A_{t}^{\prime}(0)$ is an anti-derivative of $h_{12}$, locally uniform existence of the limit $\lim _{t \searrow \sigma_{1}} A_{t}$ implies $\left(\mathrm{G}_{2}\right)$. If we assume that $\lim _{t \backslash \sigma_{1}} A_{t}=1$ and $\lim _{t \searrow \sigma_{1}} B_{t}=0$, then the functions $\alpha, \beta$ in (4.16) coincide with those from (4.4).

First we show a geometric lemma.

4.13 Lemma. Let $\mathfrak{h} \in \mathfrak{H}_{\frac{\pi}{2}}$. Then, for $t \in\left(\sigma_{1}, \sigma_{2}\right)$, the function $A_{t}-i B_{t}$ is of bounded type in the upper half-plane and belongs to the Hermite-Biehler class and to the Pólya class.

Proof. As usual the case when $\sigma_{1}$ is left endpoint of an indivisible interval can be treated explicitly. Let $\sigma_{+}$be the maximal number in $\left(\sigma_{1}, \sigma_{2}\right)$ such that $\left(\sigma_{1}, \sigma_{+}\right)$ is indivisible. The type of this indivisible interval is equal to $\frac{\pi}{2}$, and hence, by the construction of $\omega_{\mathfrak{h}}$ in $[\mathrm{KW} / \mathrm{V}]$, we have

$$
\omega_{\mathfrak{h}}\left(\sigma_{+} ; z\right)=\left(\begin{array}{cc}
1 & 0 \\
p(z) & 1
\end{array}\right)
$$

with some polynomial $p$. It follows that

$$
\begin{array}{lll}
A_{t}=1, & B_{t}=0, & t \in\left(\sigma_{1}, \sigma_{+}\right], \\
A_{t}=A_{\sigma_{+} t}, & B_{t}=B_{\sigma_{+} t}, & t \in\left[\sigma_{+}, \sigma_{2}\right) .
\end{array}
$$


The functions $A_{\sigma_{+} t}$ and $B_{\sigma_{+} t}$ are the solutions of the positive definite Hamiltonian system with Hamiltonian $\left.H\right|_{\left(\sigma_{+}, \sigma_{2}\right)}$ satisfying the initial condition $\left(A_{\sigma_{+}}(z), B_{\sigma_{+}}(z)\right)=(1,0)$. Hence, for $t \in\left[\sigma_{+}, \sigma_{2}\right)$, the function $A_{\sigma_{+} t}-i B_{\sigma_{+}} t$ belongs to the Hermite-Biehler class $\mathcal{H} B_{0}$, is of bounded and belongs to the Pólya class; see 2.13. For $t \in\left(\sigma_{1}, \sigma_{+}\right)$the assertion is trivial.

Assume now that $\sigma_{1}$ is not left endpoint of an indivisible interval. Fix a point $t_{0} \in\left(\sigma_{1}, \sigma_{2}\right)$ which is not inner point of an indivisible interval. Consider the isomorphism $\Xi_{t_{0}}: \mathcal{P}_{\dashv t_{0}} \rightarrow \mathfrak{K}\left(\omega_{\mathfrak{h}}\left(t_{0} ; \cdot\right)\right)$ as discussed at the end of §2.e. Moreover, denote by $\pi_{+}$and $\pi_{-}$the projections from $\mathfrak{K}\left(\omega_{\mathfrak{h}}\left(t_{0} ; \cdot\right)\right)$ onto its upper and lower components, respectively. By [Wo, Lemma 6.3 (proof, subcase 3b)] and the construction of $\Xi_{t_{0}}$ in $[\mathrm{KW} / \mathrm{V}]$ we have

$$
\Xi_{t_{0}}\left(\operatorname{span}\left\{\delta_{0}, \ldots, \delta_{\Delta+\ddot{o}-1}\right\}\right)=\operatorname{ker} \pi_{+},
$$

and, as, e.g. noted in [KW/I, Lemma 8.6],

$$
\mathfrak{P}\left(A_{t_{0}}-i B_{t_{0}}\right) \cong\left(\operatorname{ker} \pi_{+}\right)^{\perp} /\left(\operatorname{ker} \pi_{+}\right)^{\circ} .
$$

And application of Lemma $3.2(\mathrm{~d})$ to $\circlearrowleft_{\frac{\pi}{2}} \mathfrak{h}$ with $s_{0}=t_{0}$ gives (using the same notation as there)

$$
\mathcal{E}_{A}(\infty)=\operatorname{span}\left\{\delta_{0}, \ldots, \delta_{\Delta+\ddot{o}-1}\right\} .
$$

By Lemma 3.2 the space $\mathcal{E}_{A}(\infty)$ contains a non-positive subspace with dimension ind $\_\mathfrak{h}$, i.e. a maximal non-positive subspace of $\mathcal{P}_{\uparrow t_{0}}$. Applying $\Xi_{t_{0}}$ we obtain that $\left(\operatorname{ker} \pi_{+}\right)^{\perp}$ is positive semi-definite, and hence that $\mathfrak{P}\left(A_{t_{0}}-i B_{t_{0}}\right)$ is positive definite. This implies that the function $A_{t_{0}}-i B_{t_{0}}$ belongs to the HermiteBiehler class; see [KW/I, Theorem 5.3].

Since ker $\pi_{+} \neq\{0\}$, we have $\operatorname{ker} \pi_{-}=\{0\}$. Hence, by $[\mathrm{KW} / \mathrm{I}$, Proposition 10.3], the constant function 1 is associated with the space $\mathfrak{P}\left(D_{t_{0}}+i C_{t_{0}}\right)$. This implies that $D_{t_{0}}$ and $C_{t_{0}}$ are of bounded type in the upper half-plane; see [KW/I, $\S 2$ and $\S 3]$. Since $\frac{A_{t_{0}}}{C_{t_{0}}}$ and $\frac{B_{t_{0}}}{D_{t_{0}}}$ belong to the generalized Nevanlinna class $\mathcal{N}_{<\infty}$, and thus are of bounded type, this property is inherited by $A_{t_{0}}$ and $B_{t_{0}}$. By $\left[\mathrm{dB}\right.$, Problem 34], therefore $A_{t_{0}}-i B_{t_{0}}$ belongs to the Pólya class. From this one can now also easily obtain the assertion for inner points of an indivisible interval.

Next, we establish the knowledge of the asymptotics of $\omega_{\mathfrak{h}}(t ; \cdot)$ that is needed in the inductive process.

4.14 Lemma. Let $\mathfrak{h} \in \mathfrak{H}_{\frac{\pi}{2}}$ and assume additionally that

$$
\lim _{t \searrow \sigma_{1}} A_{t}(z)=1, \quad \lim _{t \searrow \sigma_{1}} B_{t}(z)=0
$$

locally uniformly on $\mathbb{C}$. For each $t_{0} \in\left(\sigma_{1}, \sigma_{2}\right)$ with $\gamma\left(t_{0}\right)<0$ (which certainly is the case whenever $t_{0}$ is sufficiently close to $\left.\sigma_{1}\right)$,

$$
\left\{\frac{C_{t}(z)}{\gamma(t) z}: t \in\left(\sigma_{1}, t_{0}\right]\right\}
$$

is a normal family. Moreover,

$$
\lim _{t \searrow \sigma_{1}} D_{t}(z)=1
$$


locally uniformly on $\mathbb{C}$. Let $\sigma_{+} \in\left[\sigma_{1}, \sigma_{2}\right)$ be the maximal number such that $\left(\sigma_{1}, \sigma_{+}\right)$is indivisible. Then

$$
\lim _{t \searrow \sigma_{+}} \frac{B_{t}(z)}{z \alpha(t)}=1
$$

Moreover,

$$
\frac{B_{t}(z) C_{t}(z)}{z^{2}}=\mathrm{O}(\alpha(t) \gamma(t)) \quad \text { as } t \searrow \sigma_{1}
$$

locally uniformly in $z$, and hence the left-hand side of (4.22) tends to 0 as $t \searrow \sigma_{1}$.

Proof. The case when $\sigma_{1}$ is left endpoint of an indivisible interval, i.e. when $\sigma_{1}>\sigma_{1}$, is again easy to settle. The type of $\left(\sigma_{1}, \sigma_{+}\right)$must be equal to $\frac{\pi}{2}$, and hence

$$
\omega_{\mathfrak{h}}(t ; z)=\left(\begin{array}{cc}
1 & 0 \\
p(z)-l(t) z & 1
\end{array}\right), \quad t \in\left(\sigma_{1}, \sigma_{+}\right),
$$

with some polynomial $p$ and some non-decreasing function $l(t)$ satisfying $\lim _{s \backslash \sigma_{1}} l(s)=-\infty$, cf. [KW/V, Proposition 4.31]. We have

$$
\gamma(t)=-C_{t}^{\prime}(0)=l(t)-p^{\prime}(0),
$$

and it follows that

$$
\lim _{t \sigma_{1}} \frac{C_{t}(z)}{\gamma(t) z}=\lim _{t \searrow \sigma_{1}} \frac{p(z)-l(t) z}{\left(l(t)-p^{\prime}(0)\right) z}=-1 ;
$$

in particular, the family (4.19) is normal. Moreover, $D_{t}=1, t \in\left(\sigma_{1}, \sigma_{+}\right)$, and hence the limit relation (4.20) is trivial. Relation (4.21) follows from Remark 4.8 and Lemma 4.9 since $\sigma_{+}$is a regular endpoint and $A_{\sigma_{+}}=1, B_{\sigma_{+}}=0$. Finally, relation (4.22) is trivial since $B_{t}(z)=0$ for $t \in\left(\sigma_{1}, \sigma_{+}\right)$.

Assume from now on that $\sigma_{1}$ is not left endpoint of an indivisible interval. Fix a point $t_{0} \in\left(\sigma_{1}, \sigma_{2}\right)$ that is not inner point of an indivisible interval and let $t \in\left(\sigma_{1}, t_{0}\right]$ be given. Then

$$
\omega_{\mathfrak{h}}(t ; z)=\omega_{\mathfrak{h}}\left(t_{0} ; z\right)\left[\omega_{\mathfrak{h}}(t ; z)^{-1} \omega_{\mathfrak{h}}\left(t_{0} ; z\right)\right]^{-1},
$$

i.e.

$$
\begin{aligned}
\left(\begin{array}{ll}
A_{t} & B_{t} \\
C_{t} & D_{t}
\end{array}\right) & =\left(\begin{array}{ll}
A_{t_{0}} & B_{t_{0}} \\
C_{t_{0}} & D_{t_{0}}
\end{array}\right)\left(\begin{array}{cc}
D_{t t_{0}} & -B_{t t_{0}} \\
-C_{t t_{0}} & A_{t t_{0}}
\end{array}\right) \\
& =\left(\begin{array}{ll}
A_{t_{0}} D_{t t_{0}}-B_{t_{0}} C_{t t_{0}} & -A_{t_{0}} B_{t t_{0}}+B_{t_{0}} A_{t t_{0}} \\
C_{t_{0}} D_{t t_{0}}-D_{t_{0}} C_{t t_{0}} & -C_{t_{0}} B_{t t_{0}}+D_{t_{0}} A_{t t_{0}}
\end{array}\right) .
\end{aligned}
$$

In particular,

$$
\frac{C_{t}(z)}{\gamma(t) z}=\frac{C_{t_{0}}(z)}{z} \cdot \frac{D_{t t_{0}}(z)}{\gamma(t)}-D_{t_{0}}(z) \frac{C_{t t_{0}}(z)}{\left(\gamma(t)-\gamma\left(t_{0}\right)\right) z}\left(1-\frac{\gamma\left(t_{0}\right)}{\gamma(t)}\right) .
$$

As we have noted in Remark 4.12, our assumption (4.18) implies that $H$ satisfies $\left(\mathrm{G}_{2}\right)$. Thus the application of Lemma 4.10 is justified, which implies that the 
first and second derivatives evaluated at $z=0$ of the terms on the right-hand side of (4.24) are bounded when $t \searrow \sigma_{1}$. Moreover,

$$
\left.\frac{C_{t}(z)}{\gamma(t) z}\right|_{z=0}=\frac{C_{t}^{\prime}(0)}{\gamma(t)}=-1
$$

by (4.17). Hence $-\frac{C_{t}(z)}{\gamma(t) z} \in \mathbb{P}_{c}$ for some positive $c$ and therefore the family in (4.19) is normal by Lemma 4.4.

Consider the family $\left(A_{t}(z), B_{t}(z)\right), t \in\left(\sigma_{1}, \sigma_{2}\right)$. By Lemma 4.13 , the function $A_{t}-i B_{t}$ is of bounded type in the upper half-plane and belongs to the Hermite-Biehler class $\mathcal{H} B_{0}$ and to the Pólya class. Clearly, $\left(A_{t}, B_{t}\right)$ is a solution of the canonical system with Hamiltonian $H$. We see that $\left(A_{t}, B_{t}\right)$ shares the properties (a), (b) and (c) of $\left(\AA_{t}, \stackrel{\circ}{B}_{t}\right)$ in 4.7 . By the uniqueness part of [dB, Theorem 41] it follows that

$$
A_{t}=\stackrel{\circ}{A}_{t}, \quad B_{t}=\stackrel{\circ}{B}_{t}, \quad t \in\left(\sigma_{1}, \sigma_{2}\right) .
$$

The limit in (4.21) is now nothing else but the limit computed in Lemma 4.9. Moreover, using (4.21) and the local boundedness of the left-hand side of (4.24) we find that

$$
\frac{B_{t}(z) C_{t}(z)}{z^{2}}=\alpha(t) \gamma(t) \cdot \frac{B_{t}(z)}{z \alpha(t)} \cdot \frac{C_{t}(z)}{z \gamma(t)}=\mathrm{O}(\alpha(t) \gamma(t))
$$

as $t \searrow \sigma_{1}$. This together with Lemma 4.3 implies that $B_{t}(z) C_{t}(z) \rightarrow 0$ as $t \searrow \sigma_{1}$. Since $A_{t} D_{t}-B_{t} C_{t}=1$, it follows that $\lim _{t \searrow \sigma_{1}} D_{t}=1$.

\section{d. The inductive process.}

In the following let $\mathfrak{h}$ be a singular general Hamiltonian that is either positive definite or indefinite from the class $\mathfrak{H}_{\frac{\pi}{2}}$. As above write

$$
\omega_{\mathfrak{h}}(t ; z)=:\left(\begin{array}{ll}
A_{t}(z) & B_{t}(z) \\
C_{t}(z) & D_{t}(z)
\end{array}\right) .
$$

With this notation the conditions $\left(\lim _{\frac{\pi}{2}}\right)$ and $\left(\lim _{\frac{\pi}{2}}^{\prime}\right)$ read as follows

$$
\begin{array}{ll}
\left(\lim _{\frac{\pi}{2}}\right) & \lim _{t \sigma_{1}} A_{t}(z)=\lim _{t \searrow \sigma_{1}} D_{t}(z)=1, \quad \lim _{t \searrow \sigma_{1}} B_{t}(z)=0 ; \\
\left(\lim _{\frac{\pi}{2}}^{\prime}\right) & \lim _{t \searrow \sigma_{1}} \frac{C_{t}(z)}{|\alpha(t)+\gamma(t)| \cdot z}=1,
\end{array}
$$

where $\alpha, \beta$ and $\gamma$ are as in (4.16). If $\mathfrak{h}$ is indefinite, then $q_{\mathfrak{h}} \in \mathcal{N}_{<\infty}^{(0)}$; otherwise $q_{\mathfrak{h}} \in \mathcal{N}_{0}$.

We shall apply the same inductive process as in [KW/II]. To this end it is necessary to investigate the transformations of chains employed in this process. In the following four lemmas we give the necessary supplements to the results in $[\mathrm{KW} / \mathrm{II}, \S 10]$.

4.15 Lemma. Let $\mathfrak{h}$ be a singular general Hamiltonian that is either positive definite or indefinite from the class $\mathfrak{H}_{\frac{\pi}{2}}$ satisfying $\left(\lim _{\frac{\pi}{2}}\right)$. Then

$$
\text { ind }_{-}\left(\frac{1}{z^{2}} q_{\mathfrak{h}}(z)\right)=\text { ind }_{-} q_{\mathfrak{h}}(z)+1 .
$$


Let $\tilde{\mathfrak{h}}$ be the singular general Hamiltonian with Titchmarsh-Weyl coefficient $\frac{1}{z^{2}} q_{\mathfrak{h}}(z)$. Then $\tilde{\mathfrak{h}}$ is indefinite, belongs to $\mathfrak{H}_{\frac{\pi}{2}}$ and satisfies $\left(\lim _{\frac{\pi}{2}}\right)$ and $\left(\lim _{\frac{\pi}{2}}^{\prime}\right)$.

Proof. Set $\kappa:=$ ind $_{-} \mathfrak{h}$. The function $\frac{1}{z^{2}} q_{\mathfrak{h}}(z)$ is a generalized Nevanlinna function, and ind $-\frac{1}{z^{2}} q_{\mathfrak{h}}(z) \leq \kappa+1$. However, we have

$$
\lim _{z \rightarrow i \infty} z^{2 \kappa+1} \cdot \frac{1}{z^{2}} q_{\mathfrak{h}}(z)=\lim _{z \rightarrow i \infty} z^{2 \kappa-1} \cdot q_{\mathfrak{h}}(z) \in[0, \infty)
$$

if $\mathfrak{h}$ is positive definite this is trivial, otherwise it follows from Theorem 3.1 and Remark 2.4. Thus $\frac{1}{z^{2}} q_{\mathfrak{h}}(z)$ has a generalized zero of non-positive type with degree of non-positivity at least $\kappa+1$ at $\infty$, and therefore ind $-\frac{1}{z^{2}} q_{\mathfrak{h}} \geq \kappa+1$.

Let $\tilde{\mathfrak{h}}$ be the singular general Hamiltonian with $q_{\tilde{\mathfrak{h}}}(z)=\frac{1}{z^{2}} q_{\mathfrak{h}}(z)$. By Theorem 3.1 , we already know that $\tilde{\mathfrak{h}} \in \mathfrak{H}_{\frac{\pi}{2}}$. We compute the maximal chain $\omega_{\tilde{\mathfrak{h}}}$ explicitly and then read off the required properties. Set $\alpha_{t}:=-\beta(t) \alpha(t)-\frac{1}{2} B_{t}^{\prime \prime}(0)$ and let $s_{0} \in\left[\sigma_{1}, \sigma_{2}\right)$ (or $s_{0} \in\left[\sigma_{0}, \sigma_{1}\right)$ if $\kappa=0$ ) be maximal such that $\left(\sigma_{1}, s_{0}\right)$ (or $\left(\sigma_{0}, s_{0}\right)$, respectively) is indivisible of type $\frac{\pi}{2}$. Define a function $\tilde{\omega}$ by

$$
\tilde{\omega}(t ; z):=\left\{\begin{array}{cc}
\left(\begin{array}{cc}
\frac{1}{z^{2}} & 0 \\
0 & 1
\end{array}\right) \omega_{\mathfrak{h}}(t ; z)\left(\begin{array}{cc}
0 & -\alpha(t) z \\
\frac{z}{\alpha(t)} & 1+\frac{\alpha_{t}}{\alpha(t)} z
\end{array}\right), & t \in\left(s_{0}, \sigma_{2}\right), \\
{\left[\lim _{t \nearrow \sigma_{2}} \tilde{\omega}(t ; z)\right] \cdot\left(\begin{array}{cc}
1 & \left(\frac{1}{\sigma_{2}+1-t}-1\right) z \\
0 & 1
\end{array}\right),} & t \in\left(\sigma_{2}, \sigma_{2}+1\right) \\
& \text { if } \lim _{t \nearrow \sigma_{2}} \tilde{\omega}(t ; z) \text { exists }
\end{array}\right.
$$

if $\kappa>0$, and in the same way with $\sigma_{2}$ replaced by $\sigma_{1}$ if $\kappa=0$.

It was shown in $\left[\mathrm{KW} / \mathrm{II}\right.$, Lemma 10.5] that the chain $(\tilde{\omega}(t ; z))_{t>s_{0}}$ is an end section of $\omega_{\tilde{\mathfrak{h}}}$. Computing $\tilde{\omega}(t ; z)$ explicitly for $t \in\left(s_{0}, \sigma_{2}\right)\left(t \in\left(s_{0}, \sigma_{1}\right)\right.$, respectively) gives

$$
\tilde{\omega}(t ; z)=\left(\begin{array}{cc}
\frac{B_{t}(z)}{z \alpha(t)} & -\alpha(t) \frac{A_{t}(z)}{z}+\frac{B_{t}(z)}{z^{2}}+\frac{\alpha_{t}}{\alpha(t)} \frac{B_{t}(z)}{z} \\
\frac{z D_{t}(z)}{\alpha(t)} & -\alpha(t) z C_{t}(z)+D_{t}(z)+\frac{\alpha_{t}}{\alpha(t)} z D_{t}(z)
\end{array}\right) .
$$

Let notation involving a tilde have its obvious meaning. Differentiating $\tilde{C}_{t}$ with respect to $z$, we find that $\tilde{\gamma}(t)=-\frac{1}{\alpha(t)}$. Hence

$$
\lim _{t \searrow s_{0}} \mathfrak{t}_{\tilde{\mathfrak{h}}}(t)=\lim _{t \searrow s_{0}}(\tilde{\alpha}(t)+\tilde{\gamma}(t))=-\infty
$$

since $\tilde{\alpha}(t)$ is non-decreasing, and thus $(\tilde{\omega}(t ; z))_{t>s_{0}}$ exhausts the last connected component of the chain $\omega_{\tilde{\mathfrak{h}}}$. Moreover,

$$
\lim _{t \searrow s_{0}} \tilde{\omega}(t ; z) \star \infty=\lim _{t \searrow s_{0}} \frac{B_{t}(z)}{z^{2} D_{t}(z)}=0
$$

by the assumption ( $\lim _{\frac{\pi}{2}}$ ) for $\mathfrak{h}$, and hence the intermediate Weyl coefficient of $\omega_{\tilde{\mathfrak{h}}}$ at the singularity located at the infimum of the last connected component of 
its domain is equal to 0 . It follows that the part of $\omega_{\tilde{\mathfrak{h}}}$ to the left of its singularity is just one indivisible interval of type $\frac{\pi}{2}$ and infinite length.

Referring to Lemma 4.14 (or Lemma 4.9 in the case when $\kappa=0$ ) we obtain

$$
\begin{aligned}
& \lim _{t \searrow s_{0}} \tilde{A}_{t}(z)=\lim _{t \searrow s_{0}} \frac{B_{t}(z)}{z \alpha(t)}=1, \\
& \lim _{t \searrow s_{0}} \frac{\alpha_{t}}{\alpha(t)}=\lim _{t \searrow s_{0}}\left(-\beta(t)-\left.\left(\frac{B_{t}(z)}{z \alpha(t)}\right)^{\prime}\right|_{z=0}\right)=0 .
\end{aligned}
$$

From this we get $\lim _{t \backslash s_{0}} \tilde{B}_{t}(z)=0$. Using $\lim _{t \searrow s_{0}} \alpha(t) \gamma(t)=0$ and the fact that the family in (4.19) is a normal family, we see that

$$
\lim _{t\rangle_{0}} \tilde{D}_{t}(z)=\lim _{t\rangle_{0}}\left(-\alpha(t) \gamma(t) z^{2} \frac{C_{t}(z)}{\gamma(t) z}+D_{t}(z)+\frac{\alpha_{t}}{\alpha(t)} z D_{t}(z)\right)=1 .
$$

Hence $\tilde{\mathfrak{h}}$ satisfies $\left(\lim _{\frac{\pi}{2}}\right)$, which by Remark 4.12 implies that $\lim _{t \searrow} \tilde{\alpha}(t)=0$. Finally,

$$
\frac{\tilde{C}_{t}(z)}{\tilde{\gamma}(t) z}=-D_{t}(z)
$$

and hence

$$
\lim _{t \searrow s_{0}} \frac{\tilde{C}_{t}(z)}{\left|\mathfrak{t}_{\mathfrak{h}}(t)\right| z}=\lim _{t \searrow s_{0}} \frac{\tilde{C}_{t}(z)}{|\tilde{\alpha}(t)+\tilde{\gamma}(t)| z}=\lim _{t \searrow s_{0}} \frac{\tilde{C}_{t}(z)}{-\tilde{\gamma}(t) z}=1,
$$

which is $\left(\lim _{\frac{\pi}{2}}^{\prime}\right)$ for $\tilde{\mathfrak{h}}$.

4.16 Lemma. Let $\mathfrak{h}$ be a singular general Hamiltonian which is either positive definite or indefinite from the class $\mathfrak{H}_{\frac{\pi}{2}}$ satisfying $\left(\lim _{\frac{\pi}{2}}\right)$. Then

$$
\text { ind }_{-}\left(\frac{1}{z^{2}+1} q_{\mathfrak{h}}(z)\right)=\operatorname{ind}_{-} q_{\mathfrak{h}}(z)+1 .
$$

Let $\tilde{\mathfrak{h}}$ be the singular general Hamiltonian with Titchmarsh-Weyl coefficient $\frac{1}{z^{2}+1} q_{\mathfrak{h}}(z)$. Then $\tilde{\mathfrak{h}}$ is indefinite, belongs to $\mathfrak{H}_{\frac{\pi}{2}}$ and satisfies $\left(\lim _{\frac{\pi}{2}}\right)$ and $\left(\lim _{\frac{\pi}{2}}^{\prime}\right)$.

Proof. Set $\kappa:=$ ind $\_\mathfrak{h}$. For the same reason as in the proof of Lemma 4.15 the function $\frac{1}{z^{2}+1} q_{\mathfrak{h}}(z)$ is a generalized Nevanlinna function with negative index $\kappa+1$, and $\tilde{\mathfrak{h}} \in \mathfrak{H}_{\frac{\pi}{2}}$.

Again let us compute the maximal chain $\omega_{\tilde{\mathfrak{h}}}$ explicitly. Let $s_{0} \in\left[\sigma_{1}, \sigma_{2}\right)$ (or $s_{0} \in\left[\sigma_{0}, \sigma_{1}\right)$ if $\kappa=0$ ) be maximal such that $\left(\sigma_{1}, s_{0}\right)$ (or $\left(\sigma_{0}, s_{0}\right)$, respectively) is indivisible of type $\frac{\pi}{2}$. Set $R_{t}:=\operatorname{Re} \frac{B_{t}(i)}{A_{t}(i)}$ and $J_{t}:=\operatorname{Im} \frac{B_{t}(i)}{A_{t}(i)}$. Note that $J_{t}>0$ for $t>s_{0}$ since for such values of $t$ the function $\frac{B_{t}}{A_{t}}$ is a non-constant positive definite Nevanlinna function (i.e. from the class $\mathcal{N}_{0}$ ) by Lemma 4.13, cf. 2.13. Define a function $\tilde{\omega}$ by the following formulae: if $\kappa>0$, let

$$
\tilde{\omega}(t ; z):= \begin{cases}\left(\begin{array}{cc}
\frac{1}{z^{2}+1} & 0 \\
0 & 1
\end{array}\right) \omega_{\mathfrak{h}}(t ; z)\left(\begin{array}{cc}
1-z \frac{R_{t}}{J_{t}} & -z J_{t}\left(1+\frac{R_{t}^{2}}{J_{t}^{2}}\right) \\
\frac{z}{J_{t}} & 1+z \frac{R_{t}}{J_{t}}
\end{array}\right), & t \in\left(s_{0}, \sigma_{2}\right), \\
{\left[\lim _{t>\sigma_{2}} \tilde{\omega}(t ; z)\right] \cdot W_{(l(t), \phi)}(z),} & t \in\left(\sigma_{2}, \sigma_{2}+1\right) \\
& \text { if } \lim _{t>\sigma_{2}} \tilde{\omega}(t ; z) \text { exists }\end{cases}
$$


where $l(t):=\frac{1}{\sigma_{2}+1-t}-1, \phi:=-\operatorname{Arccot}\left(\lim _{t} \sigma_{2} R_{t}\right)$ and $W_{(l, \phi)}$ was defined in (2.10); if $\kappa=0$, use the same formulae with $\sigma_{2}$ replaced by $\sigma_{1}$.

We know from [KW/II, Lemma 10.8] that the chain $(\tilde{\omega}(t ; z))_{t>s_{0}}$ is an end section of $\omega_{\tilde{\mathfrak{h}}}$. Let us again compute $\tilde{\omega}(t ; z)$ explicitly for $t \in\left(s_{0}, \sigma_{2}\right)$ :

$\tilde{\omega}(t ; z)=\left(\begin{array}{cc}\frac{1}{z^{2}+1}\left[\left(1-z \frac{R_{t}}{J_{t}}\right) A_{t}(z)+\frac{z}{J_{t}} B_{t}(z)\right] & \frac{1}{z^{2}+1}\left[\begin{array}{r}-z J_{t}\left(1+\frac{R_{t}^{2}}{J_{t}^{2}}\right) A_{t}(z)+ \\ +\left(1+z \frac{R_{t}}{J_{t}}\right) B_{t}(z)\end{array}\right] \\ \left(1-z \frac{R_{t}}{J_{t}}\right) C_{t}(z)+\frac{z}{J_{t}} D_{t}(z) & -z J_{t}\left(1+\frac{R_{t}}{J_{t}}\right) C_{t}(z)+\left(1+z \frac{R_{t}}{J_{t}}\right) D_{t}(z)\end{array}\right)$.

From the assumption $\left(\lim _{\frac{\pi}{2}}\right)$, Lemma 4.14 (in the case when $\mathfrak{h}$ is indefinite), Remark 4.8 and Lemma 4.9 (in the case when $\mathfrak{h}$ is definite) it follows that

$$
\lim _{t \searrow \sigma_{1}} \frac{B_{t}(z)}{\alpha(t) A_{t}(z)}=z
$$

which implies that $\lim _{t \searrow \sigma_{1}} \frac{1}{\alpha(t)}\left(R_{t}+i J_{t}\right)=i$ and hence

$$
\lim _{t \searrow \sigma_{1}} \frac{R_{t}}{\alpha(t)}=0, \quad \lim _{t \sigma_{1}} \frac{J_{t}}{\alpha(t)}=1, \quad \lim _{t \searrow \sigma_{1}} \frac{R_{t}}{J_{t}}=0 .
$$

If we differentiate $\tilde{C}_{t}$ with respect to $z$ and set $z=0$, we obtain

$$
\tilde{\gamma}(t)=\gamma(t)-\frac{1}{J_{t}} .
$$

Hence $\lim _{t \searrow \sigma_{1}} \mathfrak{t}_{\tilde{\mathfrak{h}}}(t)=-\infty$, and we conclude that $(\tilde{\omega}(t ; z))_{t>\sigma_{1}}$ exhausts the last connected component of the chain $\omega_{\tilde{\mathfrak{h}}}$. The relations in (4.25) imply that $\lim _{t \searrow \sigma_{1}} \tilde{B}_{t}(z)=0$. We can write

$$
\tilde{D}_{t}=-z^{2} \alpha(t) \gamma(t) \frac{J_{t}}{\alpha(t)}\left(1+\frac{R_{t}}{J_{t}}\right) \frac{C_{t}}{\gamma(t) z}+\left(1+z \frac{R_{t}}{J_{t}}\right) D_{t} .
$$

This together with Lemma 4.3, Lemma 4.14 and the relations in (4.25) implies that $\lim _{t \searrow \sigma_{1}} \tilde{D}_{t}(z)=1$. In particular, the intermediate Weyl coefficient of $\omega_{\tilde{h}}$ at $\sigma_{1}$ is equal to 0 . Next, we compute

$$
\begin{aligned}
\lim _{t \searrow \sigma_{1}}\left(1+z^{2}\right) \tilde{A}_{t}(z) & =\lim _{t \searrow \sigma_{1}}\left[\left(1-z \frac{R_{t}}{J_{t}}\right) A_{t}(z)+\frac{z}{J_{t}} B_{t}(z)\right] \\
& =\lim _{t \searrow \sigma_{1}}\left[A_{t}(z)-z \frac{R_{t}}{J_{t}} A_{t}(z)+z^{2} \frac{\alpha(t)}{J_{t}} \frac{B_{t}(z)}{z \alpha(t)}\right]=1+z^{2} .
\end{aligned}
$$

By analyticity, thus $\lim _{t \backslash \sigma_{1}} \tilde{A}_{t}(z)=1$. As in the previous lemma we therefore have $\lim _{t \backslash \sigma_{1}} \tilde{\alpha}(t)=0$ by Remark 4.12. Finally, using (4.26), (4.25), Lemma 4.3 and Lemma 4.14 we obtain

$$
\begin{aligned}
& \lim _{t \searrow \sigma_{1}} \frac{\tilde{C}_{t}(z)}{\left|\mathfrak{t}_{\tilde{\mathfrak{h}}}(t)\right| z}=\lim _{t \searrow \sigma_{1}} \frac{1}{\left|\tilde{\alpha}(t)+\gamma(t)-\frac{1}{J_{t}}\right| z}\left[\left(1-z \frac{R_{t}}{J_{t}}\right) C_{t}(z)+\frac{z}{J_{t}} D_{t}(z)\right] \\
& =\lim _{t \searrow \sigma_{1}} \frac{1}{\left|\alpha(t) \tilde{\alpha}(t)+\alpha(t) \gamma(t)-\frac{\alpha(t)}{J_{t}}\right|}\left[\alpha(t) \gamma(t)\left(1-z \frac{R_{t}}{J_{t}}\right) \frac{C_{t}(z)}{\gamma(t) z}+\frac{\alpha(t)}{J_{t}} D_{t}(z)\right] \\
& =1,
\end{aligned}
$$

which shows that $\left(\lim _{\frac{\pi}{2}}^{\prime}\right)$ is valid for $\tilde{\mathfrak{h}}$. 
4.17 Lemma. Let $\mathfrak{h}$ be a singular general Hamiltonian which is either positive definite or indefinite from the class $\mathfrak{H}_{\frac{\pi}{2}}$ satisfying $\left(\lim _{\frac{\pi}{2}}\right)$, $\left(\lim _{\frac{\pi}{2}}^{\prime}\right)$. Moreover, let $a \in \mathbb{R}$. Then ind $-q_{\mathfrak{h}}(z+a)=$ ind $_{-} q_{\mathfrak{h}}(z)$.

Let $\tilde{\mathfrak{h}}$ be the singular general Hamiltonian with Titchmarsh-Weyl coefficient $q_{\mathfrak{h}}(z+a)$; then ind $_{-} \tilde{\mathfrak{h}}=\operatorname{ind}_{-} \mathfrak{h}$. If $\mathfrak{h}$ is indefinite, then $\tilde{\mathfrak{h}}$ belongs to $\mathfrak{H}_{\frac{\pi}{2}}$ and satisfies $\left(\lim _{\frac{\pi}{2}}\right),\left(\lim _{\frac{\pi}{2}}^{\prime}\right)$.

Proof. The fact that ind $q_{\mathfrak{h}}(z+a)=\operatorname{ind}_{-} q_{\mathfrak{h}}(z)$ is trivial. Hence, in the case that $\mathfrak{h}$ is positive definite, there is nothing to prove.

Consider the case when ind $\mathfrak{h}_{-}>0$. The multiplicity of $\infty$ as a generalized zero of non-positive type of the function $q_{\mathfrak{h}}(z+a)$ is the same as its multiplicity of $q_{\mathfrak{h}}$. Thus $\tilde{\mathfrak{h}} \in \mathfrak{H}_{\frac{\pi}{2}}$.

We claim that on the last component of its domain the maximal chain $\omega_{\tilde{h}}$ is (up to a reparameterization) given by

$$
\tilde{\omega}(t ; z):=\omega_{\mathfrak{h}}(t ; z+a) \omega_{\mathfrak{h}}(t ; a)^{-1}, \quad t \in\left(\sigma_{1}, \sigma_{2}\right) .
$$

The facts that all matrices $\tilde{\omega}(t ; \cdot)$ appear in the last component of the chain $\omega_{\tilde{\mathfrak{h}}}$ and that $\lim _{t \nearrow \sigma_{2}} \mathfrak{t}_{\tilde{\mathfrak{h}}}(t)=+\infty$ have been shown in [KW/II, Lemma 10.2]. In order to see that $\tilde{\omega}$ exhausts the last component of $\omega_{\tilde{h}}$, it is sufficient to show

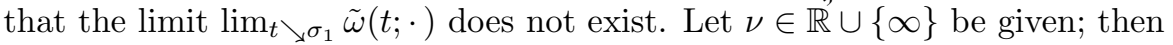

$$
\tilde{\omega}(t ; z) \star \nu=\omega_{\mathfrak{h}}(t ; z+a) \star\left(\omega_{\mathfrak{h}}(t ; a)^{-1} \star \nu\right) .
$$

Since $\omega_{\mathfrak{h}}(t ; a)^{-1} \star \nu \in \mathbb{R} \cup\{\infty\}$,

$$
\lim _{t \searrow \sigma_{1}} \tilde{\omega}(t ; z) \star \nu=0 .
$$

In particular, it does not depend on the value of $\nu$; thus $\tilde{\mathfrak{h}}$ is in the limit point case and therefore the $\operatorname{limit}_{\lim _{t} \sigma_{1}} \tilde{\omega}(t ; \cdot)$ cannot exist. We also see that the intermediate Weyl coefficient at $\sigma_{1}$ is equal to 0 , and conclude that left of $\sigma_{1}$ the chain $\omega_{\tilde{\mathfrak{h}}}$ consists of just one indivisible interval of type $\frac{\pi}{2}$.

Explicitly, $\tilde{\omega}(t ; z)$ equals

$$
\left(\begin{array}{ll}
D_{t}(a) A_{t}(z+a)-C_{t}(a) B_{t}(z+a) & -B_{t}(a) A_{t}(z+a)+A_{t}(a) B_{t}(z+a) \\
D_{t}(a) C_{t}(z+a)-C_{t}(a) D_{t}(z+a) & -B_{t}(a) C_{t}(z+a)+A_{t}(a) D_{t}(z+a)
\end{array}\right)
$$

for $t \in\left(\sigma_{1}, \sigma_{2}\right)$. Our assumption that $\mathfrak{h}$ satisfies $\left(\lim _{\frac{\pi}{2}}\right)$ immediately implies that $\lim _{t \searrow \sigma_{1}} \tilde{B}_{t}=0$. By Lemma 4.14 applied to $\mathfrak{h}$, we have

$$
\lim _{t \searrow \sigma_{1}} C_{t}(a) B_{t}(z+a)=0, \quad \lim _{t \searrow \sigma_{1}} B_{t}(a) C_{t}(z+a)=0,
$$

and hence

$$
\lim _{t \searrow \sigma_{1}} \tilde{A}_{t}(z)=\lim _{t \searrow \sigma_{1}} \tilde{D}_{t}(z)=1
$$

We need to consider $\tilde{C}_{t}(z)$. First note that $\lim _{t \searrow \sigma_{1}} \frac{C_{t}(z)}{z \gamma(t)}=-1$ (which his true by the assumption $\left.\left(\lim _{\frac{\pi}{2}}^{\prime}\right)\right)$ implies that $\lim _{t \searrow \sigma_{1}} \frac{C_{t}(z)}{\gamma(t)}=-z$, and hence $\lim _{t \searrow \sigma_{1}} \frac{C_{t}^{\prime}(z)}{\gamma(t)}=-1$. Moreover, $\frac{C_{t}(a)}{\gamma(t)}$ is bounded by Lemma 4.14, and $\lim _{t \searrow \sigma_{1}} D_{t}^{\prime}(t)=0$ because of the assumption $\left(\lim _{\frac{\pi}{2}}\right)$. Hence

$$
\lim _{t \searrow \sigma_{1}} \frac{\tilde{\gamma}(t)}{\gamma(t)}=\lim _{t \searrow \sigma_{1}} \frac{-C_{t}^{\prime}(0)}{\gamma(t)}=\lim _{t \searrow \sigma_{1}}\left[-D_{t}(a) \frac{C_{t}^{\prime}(a)}{\gamma(t)}+\frac{C_{t}(a)}{\gamma(t)} D_{t}^{\prime}(a)\right]=1 .
$$


It follows that

$$
\begin{aligned}
\lim _{t \searrow \sigma_{1}} \frac{-\tilde{C}_{t}(z)}{\left|\mathfrak{t}_{\tilde{\mathfrak{h}}}(t)\right|} & =\lim _{t \searrow \sigma_{1}} \frac{\tilde{C}_{t}(z)}{\tilde{\gamma}(t)}=\lim _{t \searrow \sigma_{1}} \frac{\tilde{C}_{t}(z)}{\gamma(t)} \\
& =\lim _{t \searrow \sigma_{1}}\left[D_{t}(a) \frac{C_{t}(z+a)}{\gamma(t)}-\frac{C_{t}(a)}{\gamma(t)} D_{t}(z+a)\right] \\
& =-(z+a)+a=-z,
\end{aligned}
$$

which implies $\left(\lim _{\frac{\pi}{2}}^{\prime}\right)$ for $\tilde{\mathfrak{h}}$.

4.18 Lemma. Let $\mathfrak{h}$ be a singular general Hamiltonian which is either positive definite or indefinite from the class $\mathfrak{H}_{\frac{\pi}{2}}$ satisfying $\left(\lim _{\frac{\pi}{2}}\right)$, $\left(\lim _{\frac{\pi}{2}}^{\prime}\right)$. Moreover, let $r>0$. Then ind $_{-} q_{\mathfrak{h}}(r z)=\operatorname{ind}_{-} q_{\mathfrak{h}}(z)$.

Let $\tilde{\mathfrak{h}}$ be the singular general Hamiltonian with Titchmarsh-Weyl coefficient $q_{\mathfrak{h}}(r z)$; then $\operatorname{ind}_{-} \tilde{\mathfrak{h}}=\operatorname{ind}_{-} \mathfrak{h}$. If $\mathfrak{h}$ is indefinite, then $\tilde{\mathfrak{h}}$ belongs to $\mathfrak{H}_{\frac{\pi}{2}}$ and satisfies $\left(\lim _{\frac{\pi}{2}}\right),\left(\lim _{\frac{\pi}{2}}^{\prime}\right)$.

Proof. Again the fact that ind $q_{\mathfrak{h}}(r z)=\operatorname{ind}_{-} q_{\mathfrak{h}}(z)$ is trivial, and therefore we have nothing to prove if ind $_{-} \mathfrak{h}=0$.

Assume that ind_ $\mathfrak{h}>0$. The multiplicity of $\infty$ as a generalized zero of non-positive type of the function $q_{\mathfrak{h}}(r z)$ is the same as its multiplicity of $q_{\mathfrak{h}}$. Thus $\tilde{\mathfrak{h}} \in \mathfrak{H}_{\frac{\pi}{2}}$.

The same arguments as in Lemma 4.17 show that $\omega_{\tilde{\mathfrak{h}}}$ is, on the last component of its domain, given by

$$
\tilde{\omega}(t ; z):=\omega_{\mathfrak{h}}(t ; r z), \quad t \in\left(\sigma_{1}, \sigma_{2}\right) ;
$$

see also [KW/II, Lemma 10.1]. The required limit relations of $\omega_{\tilde{\mathfrak{h}}}$ are immediate from this formula.

Now we are ready to finish the proof of Theorem 4.1 in the case $\alpha=\frac{\pi}{2}$.

Proof (of Theorem 4.1, case $\alpha=\frac{\pi}{2}$ ). By prolonging $\mathfrak{h}$ if necessary, we may assume, without loss of generality, that $\mathfrak{h}$ is singular. Assume that it is from the class $\mathfrak{H}_{\frac{\pi}{2}}$. Then, by Theorem 3.1, $q_{\mathfrak{h}}$ has a generalized zero of non-positive type of multiplicity $\kappa:=$ ind $_{-} q_{\mathfrak{h}}$ at $\infty$. Thus, it cannot have any zeros in $\mathbb{C} \backslash \mathbb{R}$ or generalized zeros of non-positive type on $\mathbb{R}$. By the corollary in [DLLS] we have

$$
q_{\mathfrak{h}}(z)=\left[\prod_{l=1}^{\kappa}\left(z-a_{l}\right)\left(z-\overline{a_{l}}\right)\right]^{-1} \cdot q_{0}(z),
$$

where $a_{1}, \ldots, a_{\kappa}$ denote the poles of $q_{\mathfrak{h}}$ in $\mathbb{C}^{+}$and the generalized poles of nonpositive type on $\mathbb{R}$ (according to their multiplicities) and where $q_{0}$ is from $\mathcal{N}_{0}$.

We see that the function $q_{\mathfrak{h}}$ can be produced starting from $q_{0}$ (multiplied by a positive constant) and applying a finite sequence of the transformations studied above. The Hamiltonian corresponding to $q_{0}$ is positive definite, i.e. it is in limit circle case at its left endpoint and hence satisfies $\left(\lim _{\frac{\pi}{2}}\right)$ trivially. By Lemmas $4.15-4.18$ the properties $\left(\mathrm{gH}_{\text {o.s. }}\right),\left(\mathrm{gH}_{\frac{\pi}{2}}\right)$ and $\left(\lim _{\frac{\pi}{2}}\right),\left(\lim _{\frac{\pi}{2}}^{\prime}\right)$ are inherited in each step of the induction process. Thus they hold for the maximal chain associated with $q_{\mathfrak{h}}$. 
4.19 Remark. If $H$ does not start with an indivisible interval at $\sigma_{1}$, then it follows from $(4.21)$ and $\left(\lim _{\frac{\pi}{2}}^{\prime}\right)$ that

$$
\lim _{t \searrow \sigma_{1}} \frac{B_{t}(z) C_{t}(z)}{z^{2} \alpha(t) \gamma(t)}=1
$$

locally uniformly on $\mathbb{C}$.

Let us return to the fact already announced in Remark $4.12(i)$.

4.20 Corollary. Let $H$ be a Hamiltonian defined on an interval $(a, b)$ which satisfies the conditions $\left(\mathrm{I}_{0}^{-}\right),\left(\mathrm{HS}_{0}^{-}\right)$and $\Delta_{-}(H)<\infty$. Then $\left(\mathrm{G}_{2}\right)$ holds.

Proof. Set

$$
H_{0}(x):=(x-a)^{-2} \xi_{\frac{\pi}{2}} \xi_{\frac{\pi}{2}}^{T}, \quad x \in(a-1, a) ; \quad \ddot{o}:=0, b_{1}:=0, d_{j}:=0 .
$$

Since $H$ satisfies $\left(\mathrm{I}_{0}^{-}\right),\left(\mathrm{HS}_{0}^{-}\right)$and $\Delta_{-}(H)<\infty$, the data

$$
a-1, a, b, \quad H_{0}, H, \quad \ddot{o}, b_{j}, d_{j}, \quad E:=\{a-1, b\}
$$

constitutes a general Hamiltonian $\mathfrak{h} \in \mathfrak{H}_{\frac{\pi}{2}}$. By Theorem 4.1 we have $\lim _{t \searrow a} \xi_{0}^{T} \omega_{\mathfrak{h}}(t ; z) \xi_{0}=1$ locally uniformly on $\mathbb{C}$. Now $\left(\mathrm{G}_{2}\right)$ follows from Remark 4.12 (iii).

\section{e. General values of $\alpha \in[0, \pi)$.}

Let $\alpha \in[0, \pi)$ and a general Hamiltonian $\mathfrak{h} \in \mathfrak{H}_{\alpha}$ be given. It follows from Lemma 2.26 that the general Hamiltonian $\circlearrowleft_{\alpha-\frac{\pi}{2}} \mathfrak{h}$ belongs to $\mathfrak{H}_{\frac{\pi}{2}}$. By the already proved case ' $\alpha=\frac{\pi}{2}$ ' of Theorem 4.1 we have

$$
\begin{aligned}
& \lim _{t \sigma_{1}} \xi_{0}^{T} \omega_{\circlearrowleft_{\alpha-\frac{\pi}{2}}} \mathfrak{h}(t) \xi_{0}=\lim _{t \sigma_{1}} \xi_{\frac{\pi}{2}}^{T} \omega_{\circlearrowleft_{\alpha-\frac{\pi}{2}}} \mathfrak{h}(t) \xi_{\frac{\pi}{2}}=1, \\
& \lim _{t \sigma_{1}} \xi_{0}^{T} \omega_{\circlearrowleft_{\alpha-\frac{\pi}{2}} \mathfrak{h}}(t) \xi_{\frac{\pi}{2}}=0 .
\end{aligned}
$$

However,

$$
\omega_{\circlearrowleft_{\alpha-\frac{\pi}{2}} \mathfrak{h}}=\circlearrowleft_{\alpha-\frac{\pi}{2}} \omega_{\mathfrak{h}}=N_{\alpha-\frac{\pi}{2}} \omega_{\mathfrak{h}} N_{\alpha-\frac{\pi}{2}}^{*}
$$

and

$$
N_{\alpha-\frac{\pi}{2}}^{*} \xi_{0}=\xi_{\alpha-\frac{\pi}{2}}, \quad N_{\alpha-\frac{\pi}{2}}^{*} \xi_{\frac{\pi}{2}}=\xi_{\alpha}
$$

Substituting in (4.27), gives

$$
\lim _{t \searrow \sigma_{1}} \xi_{\alpha-\frac{\pi}{2}}^{T} \omega_{\mathfrak{h}}(t) \xi_{\alpha-\frac{\pi}{2}}=\lim _{t \searrow \sigma_{1}} \xi_{\alpha}^{T} \omega_{\mathfrak{h}}(t) \xi_{\alpha}=1, \quad \lim _{t \searrow \sigma_{1}} \xi_{\alpha-\frac{\pi}{2}}^{T} \omega_{\mathfrak{h}}(t) \xi_{\alpha}=0,
$$

and this is $\left(\lim _{\alpha}\right)$. The relation $\left(\lim _{\alpha}^{\prime}\right)$ follows in the same way. This concludes the proof of Theorem 4.1 also for arbitrary values of $\alpha$.

\section{f. A continuity result for general Hamiltonians of arbitrary form.}

We can deduce an interesting continuity result for the fundamental solution of an arbitrary general Hamiltonian. 
4.21 Theorem. Let $\mathfrak{h}$ be a general Hamiltonian with ind $\mathfrak{h}_{-}>0$ given by the data

$$
\sigma_{0}, \ldots, \sigma_{n+1}, \quad H_{i}, \quad \ddot{o}_{i}, b_{i j}, d_{i j}, \quad E .
$$

Moreover, let $\omega_{\mathfrak{h}}(x ; z)$ be the (finite) maximal chain associated with $\mathfrak{h}$ and set

$$
\mathfrak{t}_{\mathfrak{h}}(x):=\mathfrak{t}\left(\omega_{\mathfrak{h}}(x ; \cdot)\right), \quad \phi_{i}:=\phi_{-}\left(H_{i}\right), i=1, \ldots, n .
$$

For each $i=1, \ldots, n$, there exist real polynomials $p_{i}, q_{i}$ and real and entire functions $e_{i}, f_{i}$ such that the following statements hold.

(i) For each $i=1, \ldots, n$, the polynomials $p_{i}$ and $q_{i}$ satisfy $p_{i}(0)=q_{i}(0)=1$, have no common zeros and no real zeros. Each $p_{i}$ and $q_{i}$ has even degree which does not exceed 2 ind $-\mathfrak{h}_{\uparrow \sigma_{i}}$.

(ii) For each $i=1, \ldots, n$, the functions $e_{i}$ and $f_{i}$ satisfy $e_{i}(0)=1$ and $f_{i}(0)=$ 0 and have no common zeros. They are of bounded type in $\mathbb{C}^{+}$and belong to the Pólya class (in particular, they have no non-real zeros).

(iii) The intermediate Weyl coefficient $q_{\mathfrak{h}, \sigma_{i}}$ of $\mathfrak{h}$ at the singularity $\sigma_{i}$ is represented as the quotient

$$
q_{\mathfrak{h}, \sigma_{i}}(z)=\frac{q_{i}(z) f_{i}(z)}{p_{i}(z) e_{i}(z)}
$$

(iv) The following limit relations hold locally uniformly on $\mathbb{C}$ :

$$
\begin{aligned}
& \lim _{x \rightarrow \sigma_{i}} \xi_{\phi_{i}+\frac{\pi}{2}}^{T} \omega_{\mathfrak{h}}(x ; z) \xi_{\phi_{i}+\frac{\pi}{2}}=p_{i}(z) e_{i}(z), \\
& \lim _{x \rightarrow \sigma_{i}} \xi_{\phi_{i}}^{T} \omega_{\mathfrak{h}}(x ; z) \xi_{\phi_{i}+\frac{\pi}{2}}=q_{i}(z) f_{i}(z), \\
& \lim _{x \rightarrow \sigma_{i}} \frac{\xi_{\phi_{i}+\frac{\pi}{2}}^{T} \omega_{\mathfrak{h}}(x ; z) \xi_{\phi_{i}}}{-\mathfrak{t}_{\mathfrak{h}}(x)}=z p_{i}(z) e_{i}(z), \\
& \lim _{x \rightarrow \sigma_{i}} \frac{\xi_{\phi_{i}}^{T} \omega_{\mathfrak{h}}(x ; z) \xi_{\phi_{i}}}{-\mathfrak{t}_{\mathfrak{h}}(x)}=z q_{i}(z) f_{i}(z) .
\end{aligned}
$$

Proof. Let $i \in\{1, \ldots, n\}$ be fixed. By appropriately prolonging $\mathfrak{h}$ if necessary, we can achieve that $\mathfrak{h}$ is singular. Of course, prolonging $\mathfrak{h}$ does not influence the limits under consideration. Moreover, using rotation isomorphisms in the routine way we see that it is enough to consider the case that $\phi_{i}=0$. If $i=1$ and the interval $\left(\sigma_{0}, \sigma_{1}\right)$ is indivisible, then the assertions follow immediately from Theorem 4.1 with $p_{1}(z)=q_{1}(z)=e_{1}(z)=1, f_{1}(z)=0$; note that in this case $q_{\mathfrak{h}, \sigma_{1}}(z)=0$. Hence in the following we assume that $\mathfrak{h}_{\mathfrak{}_{\sigma_{i}}}$ is not just one indivisible interval.

Step 1: existence of limits from above.

Set

$$
\begin{array}{ll}
\tilde{H}_{0}(x):=\left(x-\sigma_{i}\right)^{-2} \xi_{\frac{\pi}{2}} \xi_{\frac{\pi}{2}}^{T}, & x \in\left(\sigma_{i}-1, \sigma_{i}\right), \\
\tilde{H}_{1}(x):=H_{i}(x), & x \in\left(\sigma_{i}, \sigma_{i+1}\right), \\
\tilde{\ddot{o}}:=0, \tilde{b}_{1}:=0, \tilde{d}_{j}:=0, &
\end{array}
$$


and choose a point $s_{0} \in\left(\sigma_{i}, \sigma_{i+1}\right)$ which is not inner point of an indivisible interval. Then the data

$$
\sigma_{i}-1, \sigma_{i}, \sigma_{i+1}, \quad \tilde{H}_{0}, \tilde{H}_{1}, \quad \tilde{\ddot{o}}, \tilde{b}_{1}, \tilde{d}_{j}, \quad E:=\left\{\sigma_{i}-1, s_{0}, \sigma_{i+1}\right\}
$$

constitutes a general Hamiltonian $\tilde{\mathfrak{h}} \in \mathfrak{H}_{\frac{\pi}{2}}$.

Choose $x_{0} \in\left(\sigma_{i}, \sigma_{i+1}\right)$, and set

$$
M(z):=\omega_{\mathfrak{h}}\left(x_{0} ; z\right) \omega_{\tilde{\mathfrak{h}}}\left(x_{0} ; z\right)^{-1},
$$

so that $\omega_{\mathfrak{h}}\left(x_{0} ; z\right)=M(z) \omega_{\tilde{\mathfrak{h}}}\left(x_{0} ; z\right)$. Since $\omega_{\mathfrak{h}}$ and $\omega_{\tilde{\mathfrak{h}}}$ both satisfy the canonical differential equation with Hamiltonian $H_{1}$ on the interval $\left(\sigma_{i}, \sigma_{i+1}\right)$, we have

$$
\omega_{\mathfrak{h}}(y ; z)^{-1} \omega_{\mathfrak{h}}(x ; z)=\omega_{\mathfrak{h}}(y ; z)^{-1} \omega_{\tilde{\mathfrak{h}}}(x ; z), \quad x, y \in\left(\sigma_{i}, \sigma_{i+1}\right) .
$$

It follows that

$$
\omega_{\mathfrak{h}}(x ; z)=M(z) \omega_{\tilde{\mathfrak{h}}}(x ; z), \quad x \in\left(\sigma_{i}, \sigma_{i+1}\right) .
$$

Explicitly, $\omega_{\mathfrak{h}}(x ; z)$ has the form

$$
\left(\begin{array}{ll}
m_{11}(z) \omega_{\tilde{\mathfrak{h}}}(x ; z)_{11}+m_{12}(z) \omega_{\tilde{\mathfrak{h}}}(x ; z)_{21} & m_{11}(z) \omega_{\tilde{\mathfrak{h}}}(x ; z)_{12}+m_{12}(z) \omega_{\tilde{\mathfrak{h}}}(x ; z)_{22} \\
m_{21}(z) \omega_{\tilde{\mathfrak{h}}}(x ; z)_{11}+m_{22}(z) \omega_{\tilde{\mathfrak{h}}}(x ; z)_{21} & m_{21}(z) \omega_{\tilde{\mathfrak{h}}}(x ; z)_{12}+m_{22}(z) \omega_{\tilde{\mathfrak{h}}}(x ; z)_{22}
\end{array}\right)
$$

where $M(z)=\left(m_{i j}(z)\right)_{i j=1}^{2}$. By Theorem 4.1 we have (locally uniformly on $\mathbb{C}$ )

$$
\begin{aligned}
\lim _{x \searrow \sigma_{i}} \omega_{\tilde{\mathfrak{h}}}(x ; z)_{11} & =\lim _{x \searrow \sigma_{i}} \omega_{\tilde{\mathfrak{h}}}(x ; z)_{22}=\lim _{x \searrow \sigma_{i}} \frac{\omega_{\tilde{\mathfrak{h}}}(x ; z)_{21}}{-\mathfrak{t}_{\tilde{\mathfrak{h}}}(x) z}=1, \\
\lim _{x \searrow \sigma_{i}} \omega_{\tilde{\mathfrak{h}}}(x ; z)_{12} & =0,
\end{aligned}
$$

and hence (again locally uniformly on $\mathbb{C}$ )

$$
\begin{array}{rlrl}
\lim _{x \searrow \sigma_{i}} \omega_{\mathfrak{h}}(x ; z)_{12}=m_{12}(z), & & \lim _{x \searrow \sigma_{i}} \omega_{\mathfrak{h}}(x ; z)_{22}=m_{22}(z), \\
\lim _{x \searrow \sigma_{i}} \frac{\omega_{\mathfrak{h}}(x ; z)_{11}}{-\mathfrak{t}_{\tilde{\mathfrak{h}}}(x)}=z m_{12}(z), & \lim _{x \searrow \sigma_{i}} \frac{\omega_{\mathfrak{h}}(x ; z)_{21}}{-\mathfrak{t}_{\tilde{\mathfrak{h}}}(x)}=z m_{22}(z) .
\end{array}
$$

Since $\mathfrak{t}_{\mathfrak{h}}$ and $\mathfrak{t}_{\mathfrak{h}}$ are both anti-derivatives of $\operatorname{tr} H$, they differ only by a constant, and hence

$$
\lim _{x \searrow \sigma_{i}} \frac{\mathfrak{t}_{\tilde{\mathfrak{h}}}(x)}{\mathfrak{t}_{\mathfrak{h}}(x)}=1 .
$$

This shows the existence of the limits in (iv) from above. It follows that the intermediate Weyl coefficient $q_{\mathfrak{h}, \sigma_{i}}$ can be computed as follows:

$$
q_{\mathfrak{h}, \sigma_{i}}(z)=\lim _{x \searrow \sigma_{i}} \omega_{\mathfrak{h}}(x ; z) \star 0=\frac{m_{12}(z)}{m_{22}(z)} .
$$

Step 2: the functions $p_{i}, q_{i}$ and $e_{i}, f_{i}$.

Since the entries of $\omega_{\mathfrak{h}}\left(x_{0} ; z\right)$ and $\omega_{\tilde{\mathfrak{h}}}(x ; z)$ are real, entire and of bounded type in $\mathbb{C}^{+}$, also the functions $m_{12}$ and $m_{22}$ possess these properties. Moreover, since 
$\operatorname{det} M(z)=1$, they cannot have common zeros. Finally, since $M(0)=I$, we have $m_{12}(0)=0$ and $m_{22}(0)=1$.

The function $q_{\mathfrak{h}, \sigma_{i}}$ belongs to the generalized Nevanlinna class $\mathcal{N}_{<\infty}$, and ind $q_{\mathfrak{h}, \sigma_{i}}=$ ind $\mathfrak{h}_{\mathfrak{h} \sigma_{i}}$. Hence the total multiplicity of poles (or zeros) of $q_{\mathfrak{h}, \sigma_{i}}$ in $\mathbb{C}^{+}$does not exceed ind $\mathfrak{h}_{\uparrow \sigma_{i}}$. Denote by $\mathfrak{d}_{m_{12}}(w)$ the multiplicity of $w$ as a zero of $m_{12}$, let $\mathfrak{d}_{m_{22}}(w)$ be defined correspondingly, and set

$$
\begin{aligned}
& p_{i}(z):=\prod_{\substack{w \in \mathbb{C}^{+} \\
m_{22}(w)=0}}\left[\left(1-\frac{z}{w}\right)\left(1-\frac{z}{\bar{w}}\right)\right]^{\mathfrak{d}_{m_{22}}(w)}, \quad e_{i}(z):=\frac{m_{22}(z)}{p_{i}(z)}, \\
& q_{i}(z):=\prod_{\substack{w \in \mathbb{C}^{+} \\
m_{12}(w)=0}}\left[\left(1-\frac{z}{w}\right)\left(1-\frac{z}{\bar{w}}\right)\right]^{\mathfrak{d}_{m_{12}}(w)}, \quad f_{i}(z):=\frac{m_{12}(z)}{q_{i}(z)} .
\end{aligned}
$$

Clearly, the polynomials $p_{i}$ and $q_{i}$ have all properties stated in $(i)$, and the functions $e_{i}$ and $f_{i}$ have the properties (ii) (Pólya class is a consequence of bounded type). The assertion (iii) is just (4.31). The limits in (iv), when $x$ approaches $\sigma_{i}$ from above, are just (4.30).

Step 3: existence of limits from below.

Set $V:=\left(\begin{array}{cc}1 & 0 \\ 0 & -1\end{array}\right)$, and

$$
\begin{array}{ll}
\check{H}_{0}(x):=\left(x+\sigma_{i}\right)^{-2} \xi_{\frac{\pi}{2}} \xi_{\frac{\pi}{2}}^{T}, & x \in\left(-\sigma_{i}-1,-\sigma_{i}\right), \\
\check{H}_{1}(x):=V H_{i-1}(-x) V, & x \in\left(-\sigma_{i},-\sigma_{i-1}\right), \\
\check{o}:=0, \check{b}_{1}:=0, \check{d}_{j}:=0 . &
\end{array}
$$

If $i>1$, choose a point $s_{0} \in\left(\sigma_{i-1}, \sigma_{i}\right)$ which is not inner point of an indivisible interval. For $i=1$, set $s_{0}:=\sigma_{0}$. Then the data

$$
-\sigma_{i}-1,-\sigma_{i},-\sigma_{i-1}, \quad \check{H}_{0}, \check{H}_{1}, \quad \check{o}, \check{b}_{1}, \check{d}_{j}, \quad E:=\left\{-\sigma_{i}-1,-s_{0},-\sigma_{i-1}\right\}
$$

constitutes a general Hamiltonian $\check{\mathfrak{h}} \in \mathfrak{H}_{\frac{\pi}{2}}$.

The functions $\omega_{\mathfrak{h}}(x ; z)$ and $V \omega_{\mathfrak{h}}(-x ; z)^{-1} V$ are both solutions of the canonical differential equation with Hamiltonian $H_{1}$. Hence, setting (with some fixed $\left.x_{0} \in\left(\sigma_{i-1}, \sigma_{i}\right)\right)$

$$
N(z):=\omega_{\mathfrak{h}}\left(x_{0} ; z\right)\left[V \omega_{\breve{\mathfrak{h}}}\left(-x_{0} ; z\right)^{-1} V\right]^{-1}
$$

we have

$$
\omega_{\mathfrak{h}}(x ; z)=N(z)\left[V \omega_{\breve{\mathfrak{h}}}(-x ; z)^{-1} V\right], \quad x \in\left(\sigma_{i-1}, \sigma_{i}\right) .
$$

Explicitly, this relation reads as

$$
\omega_{\mathfrak{h}}(x ; z)=\left(\begin{array}{ll}
n_{11}(z) \omega_{\tilde{\mathfrak{h}}}(-x ; z)_{22}+n_{12}(z) \omega_{\tilde{\mathfrak{h}}}(-x ; z)_{21} & \left.n_{11}(z) \omega_{\tilde{\mathfrak{h}}}(-x ; z)_{12}+n_{12}(z) \omega_{\tilde{\mathfrak{h}}}(-x ; z)\right)_{11} \\
n_{21}(z) \omega_{\tilde{\mathfrak{h}}}(-x ; z)_{22}+n_{22}(z) \omega_{\tilde{\mathfrak{h}}}(-x ; z)_{21} & n_{21}(z) \omega_{\tilde{\mathfrak{h}}}(-x ; z)_{12}+n_{22}(z) \omega_{\tilde{\mathfrak{h}}}(-x ; z)_{11}
\end{array}\right) .
$$

Theorem 4.1 gives

$$
\lim _{x \searrow-\sigma_{i}} \omega_{\breve{h}}(x ; z)_{11}=\lim _{x \searrow-\sigma_{i}} \omega_{\breve{\mathfrak{h}}}(x ; z)_{22}=\lim _{x \searrow-\sigma_{i}} \frac{\omega_{\breve{\mathfrak{h}}}(x ; z)_{21}}{-\mathfrak{t}_{\mathfrak{h}}(x) z}=1, \quad \lim _{x \searrow-\sigma_{i}} \omega_{\breve{\mathfrak{h}}}(x ; z)_{12}=0,
$$


and hence (note that $\lim _{x \searrow \sigma_{i}} \frac{\mathfrak{t}_{\mathfrak{\mathfrak { h }}}(x)}{\mathfrak{t}_{\mathfrak{h}}(-x)}=1$ ),

$$
\begin{aligned}
\lim _{x \nearrow \sigma_{i}} \omega_{\mathfrak{h}}(x ; z)_{12}=n_{12}(z), & \lim _{x \nearrow \sigma_{i}} \omega_{\mathfrak{h}}(x ; z)_{22}=n_{22}(z), \\
\lim _{x \nearrow \sigma_{i}} \frac{\omega_{\mathfrak{h}}(x ; z)_{11}}{-\mathfrak{t}_{\mathfrak{h}}(x)}=z n_{12}(z), & \lim _{x \nearrow \sigma_{i}} \frac{\omega_{\mathfrak{h}}(x ; z)_{21}}{-\mathfrak{t}_{\mathfrak{h}}(x)}=z n_{22}(z) .
\end{aligned}
$$

Step 4: equality of limits from above and below.

For the same reason as in Steps 1 and 2 above, the functions $n_{12}$ and $n_{22}$ are real, entire, of bounded type, satisfy $n_{12}(0)=0, n_{22}(0)=1$, have no common zeros, and represent the intermediate Weyl coefficient as the quotient

$$
q_{\mathfrak{h}, \sigma_{i}}(z)=\frac{n_{12}(z)}{n_{22}(z)} .
$$

Hence we may factorize

$$
n_{22}(z)=p_{i}(z) \check{e}_{i}(z), \quad n_{12}(z)=q_{i}(z) \check{f}_{i}(z),
$$

where $p_{i}$ and $q_{i}$ are the polynomials defined in Step 2, and where the functions $\check{e}_{i}$ and $\check{f}_{i}$ also have the properties formulated in $(i i)$ and $(i i i)$ for $e_{i}$ and $f_{i}$.

Because of the assumption that $\mathfrak{h}_{{ } \sigma_{i}}$ is not just an indivisible interval, the functions $e_{i}, \check{e}_{i}, f_{i}, \check{f}_{i}$ are not identically equal to zero. Since they have only real zeros, belong to the Pólya class, are real, and are of bounded type, they can be represented as the products

$$
\begin{aligned}
& e_{i}(z)=\lim _{R \rightarrow \infty} \prod_{\substack{e_{i}(x)=0 \\
|x| \leq R}}\left(1-\frac{z}{x}\right)^{\mathfrak{d}_{e_{i}}(x)}, \quad \check{e}_{i}(z)=\lim _{R \rightarrow \infty} \prod_{\substack{\check{e}_{i}(x)=0 \\
|x| \leq R}}\left(1-\frac{z}{x}\right)^{\mathfrak{d}_{\check{e}_{i}}(x)}, \\
& f_{i}(z)=z \lim _{R \rightarrow \infty} \prod_{\substack{f_{i}(x)=0 \\
|x| \leq R}}\left(1-\frac{z}{x}\right)^{\mathfrak{d}_{f_{i}}(x)}, \quad \check{f}_{i}(z)=z \lim _{R \rightarrow \infty} \prod_{\substack{\check{f}_{i}(x)=0 \\
|x| \leq R}}\left(1-\frac{z}{x}\right)^{\mathfrak{d}_{\check{f}_{i}}(x)}
\end{aligned}
$$

cf. [Le, Theorem V.11]. However, $\frac{m_{12}(z)}{m_{22}(z)}=\frac{n_{12}(z)}{n_{22}(z)}$, and hence the absence of common zeros implies that the functions $e_{i}$ and $\check{e}_{i}$, or $f_{i}$ and $\check{f}_{i}$, respectively, have the same zeros including multiplicities. Thus

$$
e_{i}=\check{e}_{i}, \quad f_{i}=\check{f}_{i},
$$

i.e. the limits in $(i v)$ from above and below coincide.

In (2.14) the intermediate Weyl coefficient $q_{\mathfrak{h}, \sigma_{i}}$ at a singularity $\sigma_{i}$ was defined as a limit for non-real $z$. In the next corollary it is shown that this limit exists also for certain real $z$ and coincides with the analytic continuation of $q_{\mathfrak{h}, \sigma_{i}}(z)$.

4.22 Corollary. Let $\mathfrak{h}$ be a general Hamiltonian and $\sigma_{i}$ be one of its singularities. Further, let $q_{\mathfrak{h}, \sigma_{i}}$ be the intermediate Weyl coefficient of $\mathfrak{h}$ at $\sigma_{i}$ and let $P$ be the set of all poles of $q_{\mathfrak{h}, \sigma_{i}}$. Then, for each $\tau \in \mathbb{R} \cup\{\infty\}$, the relation

$$
q_{\mathfrak{h}, \sigma_{i}}(z)=\lim _{x \rightarrow \sigma_{i}} \omega_{\mathfrak{h}}(x ; z) \star \tau
$$


holds on $\mathbb{C} \backslash(P \cup\{0\})$ where the limit exists locally uniformly on this set (for $\tau=\cot \left(\phi_{i}+\frac{\pi}{2}\right)$ even on $\mathbb{C} \backslash P$ where $\phi_{i}$ is as in Theorem 4.21).

Proof. For $z \neq 0$ this is an immediate consequence of the fact that the limits in Theorem $4.21(i v)$ exist locally uniformly on all of $\mathbb{C}$. Next we consider the case $z=0$. For $z=0$ the right-hand side of (4.33) is equal to

$$
\lim _{x \rightarrow \sigma_{i}} \omega_{\mathfrak{h}}(x ; 0) \star \tau=\lim _{x \rightarrow \sigma_{i}} I \star \tau=\tau .
$$

To calculate $q_{\mathfrak{h}, \sigma_{i}}(0)$, replace the part of $\mathfrak{h}$ to the right of $\sigma_{i}$ by an indivisible interval $\left(\sigma_{i}, x_{0}\right)$ of type $\phi_{i}+\frac{\pi}{2}$ which is regular at $x_{0}$. Let us call this new general Hamiltonian $\tilde{\mathfrak{h}}$. For $x \in\left(\sigma_{i}, x_{0}\right)$ we have

$$
\omega_{\tilde{\mathfrak{h}}}(x ; z)=\omega_{\tilde{\mathfrak{h}}}\left(x_{0} ; z\right) W_{\left(l(x), \phi_{i}+\frac{\pi}{2}\right)}(z)
$$

where $l(x)$ is some positive function of $x$ and $W_{(l, \phi)}(z)$ is defined in (2.10). Set $\phi^{\prime}=\phi_{i}+\frac{\pi}{2}$. Then

$$
\begin{aligned}
& \omega_{\tilde{\mathfrak{h}}}(x ; z) \star \cot \phi^{\prime}=\omega_{\tilde{\mathfrak{h}}}\left(x_{0} ; z\right) W_{\left(l(x), \phi^{\prime}\right)}(z) \star \cot \phi^{\prime} \\
& =\omega_{\tilde{\mathfrak{h}}}\left(x_{0} ; z\right) \star \frac{\left(1-l(x) z \sin \phi^{\prime} \cos \phi^{\prime}\right) \cot \phi^{\prime}+l(x) z \cos ^{2} \phi^{\prime}}{-l(x) z \sin ^{2} \phi^{\prime} \cot \phi^{\prime}+1+l(x) z \sin \phi^{\prime} \cos \phi^{\prime}} \\
& =\omega_{\tilde{\mathfrak{h}}}\left(x_{0} ; z\right) \star \cot \phi^{\prime} .
\end{aligned}
$$

Hence

$$
\begin{aligned}
q_{\mathfrak{h}, \sigma_{i}}(z) & =\lim _{x \nearrow \sigma_{i}} \omega_{\mathfrak{h}}(x ; z) \star \cot \phi^{\prime}=\lim _{x \nearrow \sigma_{i}} \omega_{\tilde{\mathfrak{h}}}(x ; z) \star \cot \phi^{\prime} \\
& =\lim _{x \searrow \sigma_{i}} \omega_{\tilde{\mathfrak{h}}}(x ; z) \star \cot \phi^{\prime}=\omega_{\tilde{\mathfrak{h}}}\left(x_{0} ; z\right) \star \cot \phi^{\prime}
\end{aligned}
$$

For $z=0$ we obtain

$$
q_{\mathfrak{h}, \sigma_{i}}(0)=\omega_{\tilde{\mathfrak{h}}}\left(x_{0} ; 0\right) \star \cot \phi^{\prime}=I \star \cot \phi^{\prime}=\cot \left(\phi_{i}+\frac{\pi}{2}\right) .
$$

Hence the relation in (4.33) with $z=0$ is valid if and only if $\tau=\cot \left(\phi_{i}+\frac{\pi}{2}\right)$.

Note that the exception of the point 0 for $\tau \neq \cot \left(\phi_{i}+\frac{\pi}{2}\right)$ in Corollary 4.22 is actually necessary as is seen from the proof.

\section{Regularized boundary values}

In this section we prove the existence of regularized boundary values and the existence of a distinguished solution for which the limit towards the singularity exists. These results are then used to calculate the fundamental solution for a given Hamiltonian in the class $\mathfrak{H}_{\alpha}$, and to determine how the Titchmarsh-Weyl coefficient changes when the parameters $\ddot{o}, b_{j}$ and $d_{j}$ are changed. For $\mathcal{P}(\mathfrak{h})$, $\Gamma(\mathfrak{h})$ and $\psi(\mathfrak{h})$ see $\S 2$.e.

5.1 Theorem (Existence of regularized boundary values). Let $\mathfrak{h} \in \mathfrak{H}_{\alpha}$ be given by the data

$$
\sigma_{0}, \sigma_{1}, \sigma_{2}, \quad H_{0}, H_{1}, \quad \ddot{o}, b_{j}, d_{j}, \quad E=\left\{s_{0}, \ldots, s_{n}\right\},
$$


with $\min \left(E \cap\left(\sigma_{1}, \sigma_{2}\right)\right)=s_{1}$. Assume that $b_{\ddot{o}+1}=0$ in the case when $\sigma_{1}$ is not left endpoint of an indivisible interval. Moreover, set $\Delta:=\Delta_{-}\left(H_{1}\right)$, and denote by $\mathfrak{w}_{l}, l \in \mathbb{N}_{0}$, the unique absolutely continuous functions on $\left(\sigma_{1}, \sigma_{2}\right)$ with

$$
\begin{aligned}
& \mathfrak{w}_{0}=\xi_{\alpha}, \quad \mathfrak{w}_{l+1}^{\prime}=J H_{1} \mathfrak{w}_{l}, l \in \mathbb{N}_{0} \\
& \mathfrak{w}_{l}\left(s_{1}\right) \in \operatorname{span}\left\{\xi_{\alpha}\right\} \\
& \left.\mathfrak{w}_{\Delta}\right|_{\left(\sigma_{1}, s_{1}\right)} \in L^{2}\left(\left.H\right|_{\left(\sigma_{1}, s_{1}\right)}\right) .
\end{aligned}
$$

Let $z \in \mathbb{C}$ and $\psi$ be a solution of the Hamiltonian system

$$
y^{\prime}(x)=z J H_{1}(x) y(x), \quad x \in\left(\sigma_{1}, \sigma_{2}\right) .
$$

Then the boundary value

$$
\operatorname{rbv}_{\mathrm{r}} \psi:=\lim _{x \searrow \sigma_{1}} \xi_{\alpha}^{T} \psi(x),
$$

and the regularized boundary value

$$
\begin{aligned}
\operatorname{rbv}_{\mathrm{s}}(z) \psi:= & \lim _{x \searrow \sigma_{1}}\left[\sum_{l=0}^{\Delta} z^{l}\left(\mathfrak{w}_{l}(x)\right)^{*} J\left(\psi(x)-\operatorname{rbv}_{\mathrm{r}} \psi \cdot \sum_{k=\Delta+1}^{2 \Delta-l} z^{k} \mathfrak{w}_{k}(x)\right)\right] \\
& +\operatorname{rbv}_{\mathrm{r}} \psi\left(\sum_{l=1}^{2 \Delta} z^{l} d_{l-1}-\sum_{l=0}^{\ddot{o}} z^{2 \Delta+l} b_{\ddot{o}+1-l}\right)
\end{aligned}
$$

exist. Set

$$
\operatorname{rbv}(z) \psi:=\operatorname{rbv}_{\mathrm{s}}(z) \psi \cdot \xi_{\alpha-\frac{\pi}{2}}+\operatorname{rbv}_{\mathrm{r}} \psi \cdot \xi_{\alpha} .
$$

For each $z \in \mathbb{C}$ and $a \in \mathbb{C}^{2}$ there exists a unique solution $\psi$ of (5.1) with $\operatorname{rbv}(z) \psi=a$.

Moreover, for given $\psi$ as above let $F$ be the unique element in $\mathcal{P}(\mathfrak{h})$ such that $(F ; z F) \in T_{\max }(\mathfrak{h})$ and $\psi(\mathfrak{h}) F=\psi$. Then

$$
\operatorname{rbv}(z) \psi=\pi_{l} \circ \Gamma(\mathfrak{h})(F ; z F) .
$$

5.2 Theorem (Existence of a distinguished solution). Let $\mathfrak{h}$ be a general Hamiltonian as in Theorem 5.1. Let $z \in \mathbb{C}$ and $\psi$ be a solution of (5.1). Then

$$
\lim _{x \searrow \sigma_{1}} \xi_{\alpha}^{T} \psi(x)=\xi_{\alpha}^{T} \operatorname{rbv}(z) \psi .
$$

For $z \neq 0$ the following are equivalent:

(i) $\lim _{x \searrow \sigma_{1}} \xi_{\alpha-\frac{\pi}{2}}^{T} \psi(x)$ exists;

(ii) $\operatorname{rbv}(z) \psi \in \operatorname{span}\left\{\xi_{\alpha-\frac{\pi}{2}}\right\}$;

(iii) $\operatorname{rbv}_{\mathrm{r}} \psi=0$;

(iv) $\left.\psi\right|_{\left(\sigma_{1}, s_{1}\right)} \in L^{2}\left(\left.H\right|_{\left(\sigma_{1}, s_{1}\right)}\right)$.

In this case,

$$
\lim _{x \searrow \sigma_{1}} \psi(x)=\operatorname{rbv}(z) \psi
$$


5.3 Remark. The case ' $z=0$ ' is indeed exceptional. For $z=0$ the right-hand side of (5.3) reduces to $\operatorname{rbv}_{\mathrm{S}}(0) \psi=\lim _{x \searrow \sigma_{1}} \xi_{\alpha-\frac{\pi}{2}}^{T} \psi(x)$ and hence

$$
\operatorname{rbv}(0) \psi=\lim _{x \searrow \sigma_{1}} \psi(x) .
$$

Therefore $(i)$ is satisfied for all solutions of (5.1) but (ii)-(iv) are satisfied only for multiples of the constant function $\xi_{\alpha-\frac{\pi}{2}}$. Note that all solutions of (5.1) with $z=0$ are constant functions.

According to condition (E2) in Definition 2.18 the interval $\left(\sigma_{1}, s_{1}\right)$ is either a maximal indivisible interval of type $\alpha$ or $\sigma_{1}$ is not left endpoint of an indivisible interval. We first settle the case when $\alpha=\frac{\pi}{2}$ and $\left(\sigma_{1}, s_{1}\right)$ is an indivisible interval.

Proof (of Theorems 5.1 and 5.2 when $\alpha=\frac{\pi}{2}$ and $\left(\sigma_{1}, s_{1}\right)$ is indivisible).

In this case we have to consider the elementary Hamiltonian on $\left(\sigma_{0}, \sigma_{1}\right) \cup\left(\sigma_{1}, s_{1}\right)$, which is of kind (B) or (C); see [KW/IV, Definition 4.1]. By this definition we have $d_{1}=0$. Since $\alpha=\frac{\pi}{2}$, we can write

$$
H_{0}(x)=\left(\begin{array}{cc}
0 & 0 \\
0 & h_{0}(x)
\end{array}\right), x \in\left(\sigma_{0}, \sigma_{1}\right) ; \quad H_{1}(x)=\left(\begin{array}{cc}
0 & 0 \\
0 & h_{1}(x)
\end{array}\right), x \in\left(\sigma_{1}, s_{1}\right),
$$

with real-valued functions $h_{0}, h_{1}$ which are locally integrable on $\left[\sigma_{0}, \sigma_{1}\right)$ and $\left(\sigma_{1}, s_{1}\right]$, respectively, but not integrable at $\sigma_{1}$. According to $[\mathrm{KW} / \mathrm{V}$, Corollary 4.32] we have

$$
\omega_{\mathfrak{h}}\left(s_{1} ; z\right)=\left(\begin{array}{cc}
1 & 0 \\
-z d_{0}+z^{2} b_{\ddot{o}+1}+\ldots+z^{\ddot{o}+2} b_{1} & 1
\end{array}\right),
$$

and with

$$
\ell_{-}(x):=\int_{\sigma_{0}}^{x} h_{0}(t) d t, \quad x \in\left[\sigma_{0}, \sigma_{1}\right), \quad \text { and } \quad \ell_{+}(x):=\int_{x}^{s_{1}} h_{1}(t) d t, \quad x \in\left(\sigma_{1}, s_{1}\right],
$$

we therefore obtain

$$
\omega_{\mathfrak{h}}(x ; z)=\left\{\begin{array}{cc}
\left(\begin{array}{cc}
1 & 0 \\
-\ell_{-}(x) & 1
\end{array}\right), & x \in\left[\sigma_{0}, \sigma_{1}\right), \\
\left(\begin{array}{cc}
1 & 0 \\
z\left(\ell_{+}(x)-d_{0}\right)+z^{2} b_{\ddot{o}+1}+\ldots+z^{\ddot{o}+2} b_{1} & 1
\end{array}\right), & x \in\left(\sigma_{1}, s_{1}\right] .
\end{array}\right.
$$

An arbitrary solution $\psi$ of (5.1) is a linear combination of the rows of $\omega_{\mathfrak{h}}$, i.e., with arbitrary $a=\left(a_{1}, a_{2}\right)^{T} \in \mathbb{C}^{2}$,

$$
\begin{aligned}
\psi(x) & =a_{1}\left(\begin{array}{l}
\omega_{\mathfrak{h}}(x ; z)_{11} \\
\omega_{\mathfrak{h}}(x ; z)_{12}
\end{array}\right)+a_{2}\left(\begin{array}{c}
\omega_{\mathfrak{h}}(x ; z)_{21} \\
\omega_{\mathfrak{h}}(x ; z)_{22}
\end{array}\right) \\
& =\left\{\begin{array}{cc}
\left(\begin{array}{c}
a_{1}-a_{2} \ell_{-}(x) \\
a_{2}
\end{array}\right), & x \in\left[\sigma_{0}, \sigma_{1}\right), \\
\left(\begin{array}{cc}
a_{1}+a_{2}\left(z\left(\ell_{+}(x)-d_{0}\right)+\sum_{l=0}^{\ddot{o}} z^{l+2} b_{\ddot{o}+1-l}\right) \\
a_{2}
\end{array}\right), & x \in\left(\sigma_{1}, s_{1}\right] .
\end{array}\right.
\end{aligned}
$$


For the calculation of the regularized boundary values we need $\mathfrak{w}_{0}, \mathfrak{w}_{1}$ and $\mathfrak{w}_{2}$; note that $\Delta=1$ since we have indivisible intervals on both sides of $\sigma_{1}$. On the interval $\left(\sigma_{1}, s_{1}\right]$ we have

$$
\mathfrak{w}_{0}(x)=\left(\begin{array}{l}
0 \\
1
\end{array}\right), \quad \mathfrak{w}_{1}(x)=\left(\begin{array}{c}
\ell_{+}(x) \\
0
\end{array}\right), \quad \mathfrak{w}_{2}(x)=0 .
$$

For $\operatorname{rbv}_{\mathrm{r}} \psi$ we obtain

$$
\operatorname{rbv}_{\mathrm{r}} \psi=\lim _{x \searrow \sigma_{1}} \psi_{2}(x)=a_{2} .
$$

The expression in (5.3) yields

$$
\begin{aligned}
\operatorname{rbv}_{\mathbf{s}}(z) \psi= & \lim _{x \searrow \sigma_{1}}\left[\mathfrak{w}_{0}(x)^{*} J\left(\psi(x)-a_{2} z^{2} \mathfrak{w}_{2}(x)\right)+z \mathfrak{w}_{1}(x)^{*} J \psi(x)\right] \\
& +a_{2}\left(z d_{0}-\sum_{l=0}^{\ddot{o}} z^{l+2} b_{\ddot{o}+1-l}\right) \\
= & \lim _{x \searrow \sigma_{1}}\left[\left(\mathfrak{w}_{0}(x)+z \mathfrak{w}_{1}(x)\right)^{*} J \psi(x)\right]+a_{2}\left(z d_{0}-\sum_{l=0}^{\ddot{o}} z^{l+2} b_{\ddot{o}+1-l}\right) \\
= & \lim _{x \searrow \sigma_{1}}\left(\psi_{1}(x)-z \ell_{+}(x) \psi_{2}(x)\right)+a_{2}\left(z d_{0}-\sum_{l=0}^{\ddot{o}} z^{l+2} b_{\ddot{o}+1-l}\right) \\
= & a_{1}-a_{2} z d_{0}+a_{2} \sum_{l=0}^{\ddot{o}} z^{l+2} b_{\ddot{o}+1-l}+a_{2}\left(z d_{0}-\sum_{l=0}^{\ddot{o}} z^{l+2} b_{\ddot{o}+1-l}\right) \\
= & a_{1} .
\end{aligned}
$$

Hence $\operatorname{rbv}(z) \psi=\left(a_{1}, a_{2}\right)^{T}$, which shows that for every $a \in \mathbb{C}^{2}$ we have a unique solution $\psi$ of $(5.1)$ with $\operatorname{rbv}(z) \psi=a$. So all assertions of Theorem 5.1 are proved (relation (5.4) follows from the very definition of $\omega_{\mathfrak{h}}$ in $[\mathrm{KW} / \mathrm{V}$, Lemma 4.1, Definition 4.3]).

Equation (5.5) in Theorem 5.2 is valid since both sides are equal to $a_{2}$; note that $\xi_{\alpha}=(0,1)^{T}$. It is easy to see that the statements $(i)$ to $(i v)$ are all equivalent to the fact that $a_{2}=0$. Finally, if $a_{2}=0$, then both sides of (5.6) are equal to $a_{1}$.

Now we turn to the proof of Theorem 5.1 in the case when $\sigma_{1}$ is not left endpoint of an indivisible interval. The core of the proof is the following lemma, where we rewrite formula (2.21) for a defect element.

5.4 Lemma. Let $\mathfrak{h}$ be a regular general Hamiltonian of the form 2.20. Let $z \in \mathbb{C}, F=(f ; \xi, \alpha) \in \stackrel{\infty}{\mathcal{P}}(\mathfrak{h})$ and assume that $(F ; z F) \in \stackrel{\infty}{T}(\mathfrak{h})$. Moreover, let $\mathfrak{w}_{l}$ be as in (2.15)-(2.17), and let $\lambda_{l}$ be the unique scalars such that

$$
\tilde{f}:=f-\sum_{l=0}^{\Delta-1} \lambda_{l} \mathfrak{w}_{l} \in L^{2}\left(\left.H\right|_{\left(\sigma_{1}, \sigma_{2}\right)}\right) .
$$


Then the limit

$$
L:=\lim _{x \searrow \sigma_{1}}\left[\sum_{l=0}^{\Delta} z^{l}\left(\mathfrak{w}_{l}(x)\right)^{*} J\left(f(x)-\lambda_{0} \sum_{k=\Delta+1}^{2 \Delta-l} z^{k} \mathfrak{w}_{k}(x)\right)\right]
$$

exists, and

$$
\pi_{l} \circ \stackrel{\infty}{\Gamma}(F ; z F)=\left(\begin{array}{c}
L+\lambda_{0}\left(\sum_{l=1}^{2 \Delta} z^{l} d_{l-1}-\sum_{l=1}^{\ddot{o}} z^{2 \Delta+l} b_{\ddot{o}+1-l}\right) \\
\lambda_{0}
\end{array}\right) .
$$

Proof. We use Proposition 2.22 and Definition 2.21 with the element $(F ; z F)$, i.e. $g=z f, \eta_{l}=z \xi_{l}, \beta_{l}=z \alpha_{l}$, and, consequently, $\mu_{l}=z \lambda_{l}$.

Step 1: computation of $\beta_{1}$.

It follows from Definition $2.21(i)$ that $\lambda_{k+1}=\mu_{k}=z \lambda_{k}, k=0, \ldots, \Delta-2$, and hence

$$
\lambda_{k}=z^{k} \lambda_{0}, \quad \mu_{k}=z^{k+1} \lambda_{0}, \quad k=0, \ldots, \Delta-1 .
$$

If $\ddot{o}>0$, we obtain from Definition $2.21(i v)$ that

$$
\begin{aligned}
& \alpha_{\ddot{o}}=z^{\Delta} \lambda_{0} b_{1}, \\
& \alpha_{j}=z^{\Delta} \lambda_{0} b_{\ddot{o}-j+1}+z \alpha_{j+1}, \quad k=1, \ldots, \ddot{o}-1 .
\end{aligned}
$$

By induction,

$$
\alpha_{k}=\lambda_{0}\left(z^{\Delta} b_{\ddot{o}-k+1}+z^{\Delta+1} b_{\ddot{o}-k}+\cdots+z^{\Delta+\ddot{o}-k} b_{1}\right), \quad k=1, \ldots, \ddot{o},
$$

and hence

$$
\beta_{1}=z \alpha_{1}=\lambda_{0} \sum_{l=1}^{\ddot{o}} z^{\Delta+l} b_{\ddot{o}+1-l} .
$$

In order to unify notation, we set $\beta_{1}:=0$ when $\ddot{o}=0$.

Step 2: computation of $\eta_{0}$.

We shall show by induction that, for $k=0, \ldots, \Delta-1$,

$$
\begin{aligned}
\xi_{k}= & z^{\Delta-k} \int_{\sigma_{1}}^{\sigma_{2}}\left(\mathfrak{w}_{\Delta}\right)^{*} H \tilde{f}+\sum_{l=0}^{\Delta-k-1} z^{l}\left(\mathfrak{w}_{k+l+1}\left(\sigma_{2}\right)\right)_{2} f\left(\sigma_{2}\right)_{1} \\
& +\lambda_{0}\left(\frac{1}{2} \sum_{l=0}^{\Delta-1} z^{l} d_{k+l}+\sum_{l=0}^{\Delta-k-1} z^{\Delta+l} d_{\Delta+l+k}-\sum_{l=1}^{\ddot{o}} z^{2 \Delta+l-k-1} b_{\ddot{o}+1-l}\right) .
\end{aligned}
$$


For $k=\Delta-1$ we obtain from Definition $2.21($ iii $)$ that

$$
\begin{aligned}
\xi_{\Delta-1}= & \int_{\sigma_{1}}^{\sigma_{2}}\left(\mathfrak{w}_{\Delta}\right)^{*} H z \tilde{f}+\frac{1}{2} \sum_{l=0}^{\Delta-1} z^{l} \lambda_{0} d_{l+\Delta-1}+z^{\Delta} \lambda_{0} d_{2 \Delta-1} \\
& +\left(\mathfrak{w}_{\Delta}\left(\sigma_{2}\right)\right)_{2} f\left(\sigma_{2}\right)_{1}-\beta_{1} \\
= & z \int_{\sigma_{1}}^{\sigma_{2}}\left(\mathfrak{w}_{\Delta}\right)^{*} H \tilde{f}+\left(\mathfrak{w}_{\Delta}\left(\sigma_{2}\right)\right)_{2} f\left(\sigma_{2}\right)_{1} \\
& +\lambda_{0}\left(\frac{1}{2} \sum_{l=0}^{\Delta-1} z^{l} d_{l+\Delta-1}+z^{\Delta} d_{2 \Delta-1}-\sum_{l=1}^{\ddot{o}} z^{\Delta+l} b_{\ddot{o}+1-l}\right)
\end{aligned}
$$

which is (5.8) for $k=\Delta-1$. Let $k \in\{0, \ldots, \Delta-2\}$ and assume that (5.8) is true for $k$ replaced by $k+1$. Then, by Definition $2.21(i i)$,

$$
\begin{aligned}
& \xi_{k}=z \xi_{k+1}+\frac{1}{2} z^{\Delta} \lambda_{0} d_{\Delta+k}+\frac{1}{2} \lambda_{0} d_{k}+\left(\mathfrak{w}_{k+1}\left(\sigma_{2}\right)\right)_{2} f\left(\sigma_{2}\right)_{1} \\
& =z^{\Delta-k} \int_{\sigma_{1}}^{\sigma_{2}}\left(\mathfrak{w}_{\Delta}\right)^{*} H \tilde{f}+\sum_{l=0}^{\Delta-k-2} z^{l+1}\left(\mathfrak{w}_{k+l+2}\left(\sigma_{2}\right)\right)_{2} f\left(\sigma_{2}\right)_{1} \\
& +\lambda_{0}\left(\frac{1}{2} \sum_{l=0}^{\Delta-1} z^{l+1} d_{k+l+1}+\sum_{l=0}^{\Delta-k-2} z^{\Delta+l+1} d_{\Delta+l+k+1}-\sum_{l=1}^{\ddot{o}} z^{2 \Delta+l-k-1} b_{\ddot{o}+1-l}\right) \\
& +\frac{1}{2} z^{\Delta} \lambda_{0} d_{\Delta+k}+\frac{1}{2} \lambda_{0} d_{k}+\left(\mathfrak{w}_{k+1}\left(\sigma_{2}\right)\right)_{2} f\left(\sigma_{2}\right)_{1} \\
& =z^{\Delta-k} \int_{\sigma_{1}}^{\sigma_{2}}\left(\mathfrak{w}_{\Delta}\right)^{*} H \tilde{f}+\sum_{l=1}^{\Delta-k-1} z^{l}\left(\mathfrak{w}_{k+l+1}\left(\sigma_{2}\right)\right)_{2} f\left(\sigma_{2}\right)_{1}+\left(\mathfrak{w}_{k+1}\left(\sigma_{2}\right)\right)_{2} f\left(\sigma_{2}\right)_{1} \\
& +\lambda_{0}\left(\frac{1}{2} \sum_{l=1}^{\Delta} z^{l} d_{k+l}+\frac{1}{2} d_{k}+\frac{1}{2} z^{\Delta} d_{\Delta+k}+\sum_{l=1}^{\Delta-k-1} z^{\Delta+l} d_{\Delta+l+k}\right. \\
& \left.-\sum_{l=1}^{\ddot{o}} z^{2 \Delta+l-k-1} b_{\ddot{o}+1-l}\right) \\
& =z^{\Delta-k} \int_{\sigma_{1}}^{\sigma_{2}}\left(\mathfrak{w}_{\Delta}\right)^{*} H \tilde{f}+\sum_{l=0}^{\Delta-k-1} z^{l}\left(\mathfrak{w}_{k+l+1}\left(\sigma_{2}\right)\right)_{2} f\left(\sigma_{2}\right)_{1} \\
& +\lambda_{0}\left(\frac{1}{2} \sum_{l=0}^{\Delta-1} z^{l} d_{k+l}+z^{\Delta} d_{\Delta+k}+\sum_{l=1}^{\Delta-k-1} z^{\Delta+l} d_{\Delta+l+k}\right. \\
& \left.-\sum_{l=1}^{\ddot{o}} z^{2 \Delta+l-k-1} b_{\ddot{o}+1-l}\right) \text {, }
\end{aligned}
$$

which is equal to the right-hand side of (5.8). Thus (5.8) holds for all $k \in$ 
$\{0, \ldots, \Delta-1\}$, and it follows that

$$
\begin{aligned}
\eta_{0}= & z \xi_{0} \\
= & z^{\Delta+1} \int_{\sigma_{1}}^{\sigma_{2}}\left(\mathfrak{w}_{\Delta}\right)^{*} H \tilde{f}+\sum_{l=0}^{\Delta-1} z^{l+1}\left(\mathfrak{w}_{l+1}\left(\sigma_{2}\right)\right)_{2} f\left(\sigma_{2}\right)_{1} \\
& +\lambda_{0}\left(\frac{1}{2} \sum_{l=0}^{\Delta-1} z^{l+1} d_{l}+\sum_{l=0}^{\Delta-1} z^{\Delta+l+1} d_{\Delta+l}-\sum_{l=1}^{\ddot{o}} z^{2 \Delta+l} b_{\ddot{o}+1-l}\right) .
\end{aligned}
$$

Step 3: first component of the left boundary value.

By (2.21) the first component of the boundary value at the left endpoint $\sigma_{0}$ is equal to

$$
\begin{aligned}
\left(\pi_{l} \circ \tilde{\Phi}(F ; z F)\right)_{1}= & \eta_{0}+f\left(\sigma_{2}\right)_{1}+\frac{1}{2} \sum_{l=0}^{\Delta-1} z^{l+1} \lambda_{0} d_{l} \\
= & z^{\Delta+1} \int_{\sigma_{1}}^{\sigma_{2}}\left(\mathfrak{w}_{\Delta}\right)^{*} H \tilde{f}+\sum_{l=0}^{\Delta} z^{l}\left(\mathfrak{w}_{l}\left(\sigma_{2}\right)\right)_{2} f\left(\sigma_{2}\right)_{1} \\
& +\lambda_{0}\left(\sum_{l=0}^{\Delta-1} z^{l+1} d_{l}+\sum_{l=0}^{\Delta-1} z^{\Delta+l+1} d_{\Delta+l}-\sum_{l=1}^{\ddot{o}} z^{2 \Delta+l} b_{\ddot{o}+1-l}\right) \\
= & z^{\Delta+1} \int_{\sigma_{1}}^{\sigma_{2}}\left(\mathfrak{w}_{\Delta}\right)^{*} H \tilde{f}+\sum_{l=0}^{\Delta} z^{l}\left(\mathfrak{w}_{l}\left(\sigma_{2}\right)\right)_{2} f\left(\sigma_{2}\right)_{1} \\
& +\lambda_{0}\left(\sum_{l=1}^{2 \Delta} z^{l} d_{l-1}-\sum_{l=1}^{\ddot{o}} z^{2 \Delta+l} b_{\ddot{o}+1-l}\right)
\end{aligned}
$$

Step 4: removing the integral term.

Take an arbitrary $x \in\left(\sigma_{1}, \sigma_{2}\right)$ and apply Green's identity (2.11) to the interval $\left(x, \sigma_{2}\right)$ :

$$
\begin{aligned}
& z^{\Delta+1} \int_{x}^{\sigma_{2}}\left(\mathfrak{w}_{\Delta}\right)^{*} H \tilde{f}+\sum_{l=0}^{\Delta} z^{l}\left(\mathfrak{w}_{l}\left(\sigma_{2}\right)\right)_{2} f\left(\sigma_{2}\right)_{1} \\
& =z^{\Delta} \int_{x}^{\sigma_{2}}\left(\mathfrak{w}_{\Delta}\right)^{*} H\left(z f-\lambda_{0} \sum_{k=0}^{\Delta-1} z^{k+1} \mathfrak{w}_{k}\right)+\sum_{l=0}^{\Delta} z^{l}\left(\mathfrak{w}_{l}\left(\sigma_{2}\right)\right)_{2} f\left(\sigma_{2}\right)_{1}
\end{aligned}
$$




$$
\begin{aligned}
= & z^{\Delta}\left[\int_{x}^{\sigma_{2}}\left(\mathfrak{w}_{\Delta-1}\right)^{*} H\left(f-\lambda_{0} \sum_{k=0}^{\Delta-1} z^{k+1} \mathfrak{w}_{k+1}\right)\right. \\
& +\left(\mathfrak{w}_{\Delta}(x)\right)^{*} J\left(f(x)-\lambda_{0} \sum_{k=0}^{\Delta-1} z^{k+1} \mathfrak{w}_{k+1}(x)\right) \\
& \left.-\left(\mathfrak{w}_{\Delta}\left(x_{0}\right)\right)^{*} J\left(f\left(\sigma_{2}\right)-\lambda_{0} \sum_{k=0}^{\Delta-1} z^{k+1} \mathfrak{w}_{k+1}\left(\sigma_{2}\right)\right)\right]+\sum_{l=0}^{\Delta} z^{l}\left(\mathfrak{w}_{l}\left(\sigma_{2}\right)\right)_{2} f\left(\sigma_{2}\right)_{1} \\
= & z^{\Delta-1} \int_{x}^{\sigma_{2}}\left(\mathfrak{w}_{\Delta-1}\right)^{*} H\left(z f-\lambda_{0} \sum_{k=0}^{\Delta-1} z^{k+2} \mathfrak{w}_{k+1}\right) \\
& +z^{\Delta}\left(\mathfrak{w}_{\Delta}(x)\right)^{*} J\left(f(x)-\lambda_{0} \sum_{k=0}^{\Delta-1} z^{k+1} \mathfrak{w}_{k+1}(x)\right)+\sum_{l=0}^{\Delta-1} z^{l}\left(\mathfrak{w}_{l}\left(\sigma_{2}\right)\right)_{2} f\left(\sigma_{2}\right)_{1} \\
= & z^{\Delta-1}\left[\int_{x}^{\sigma_{2}}\left(\mathfrak{w}_{\Delta-2}\right)^{*} H\left(f-\lambda_{0} \sum_{k=0}^{\Delta-1} z^{k+2} \mathfrak{w}_{k+2}\right)\right. \\
& +\left(\mathfrak{w}_{\Delta-1}(x)\right)^{*} J\left(f(x)-\lambda_{0} \sum_{k=0}^{\Delta-1} z^{k+2} \mathfrak{w}_{k+2}(x)\right) \\
& +z^{\Delta-1}\left(\mathfrak{w}_{\Delta-1}(x)\right)^{*} J\left(f(x)-\lambda_{0} \sum_{k=0}^{\Delta-1} z^{k+2} \mathfrak{w}_{k+2}(x)\right) \\
& \left.-\left(\mathfrak{w}_{\Delta-1}\left(\sigma_{2}\right)\right)^{*} J\left(f\left(\sigma_{2}\right)-\lambda_{0} \sum_{k=0}^{\Delta-1} z^{k+2} \mathfrak{w}_{k+2}\left(\sigma_{2}\right)\right)\right] \\
& +z^{\Delta}\left(\mathfrak{w}_{\Delta}(x)\right)^{*} J\left(f(x)-\lambda_{0} \sum_{k=0}^{\Delta-1} z^{k+1} \mathfrak{w}_{k+1}(x)\right)+\sum_{l=0}^{\Delta-1} z^{l}\left(\mathfrak{w}_{l}\left(\sigma_{2}\right)\right)_{2} f\left(\sigma_{2}\right)_{1} \\
\sigma_{2} & \left.z_{\Delta-2}\right)^{*} H\left(z f-\lambda_{0} \sum_{k=0}^{\Delta-1} z^{k+2} \mathfrak{w}_{k+2}\right) \\
& \\
& \\
& \\
& \\
&
\end{aligned}
$$

Proceeding inductively, we obtain that the expression in (5.11) equals

$$
\sum_{l=0}^{\Delta} z^{l}\left(\mathfrak{w}_{l}(x)\right)^{*} J\left(f(x)-\lambda_{0} \sum_{k=\Delta+1-l}^{2 \Delta-l} z^{k} \mathfrak{w}_{k}(x)\right) .
$$

Step 5: finishing the proof.

Since $J^{*}=-J$, we have

$$
\left(\mathfrak{w}_{l}(x)\right)^{*} J \mathfrak{w}_{k}(x)+\left(\mathfrak{w}_{k}(x)\right)^{*} J \mathfrak{w}_{l}(x)=0
$$


for $k, l \in \mathbb{N}_{0}$. Hence

$$
\begin{aligned}
& \sum_{l=0}^{\Delta} \sum_{\substack{k=\Delta+1-l \\
\Delta}}^{\Delta}\left(\mathfrak{w}_{l}(x)\right)^{*} J \mathfrak{w}_{k}(x)=\sum_{\substack{1 \leq k, l \leq \Delta \\
\Delta+1 \leq k+l \leq 2 \Delta}}\left(\mathfrak{w}_{l}(x)\right)^{*} J \mathfrak{w}_{k}(x) \\
& =\frac{1}{2} \sum_{\substack{1 \leq k, l \leq \Delta \\
\Delta+1 \leq k+l \leq 2 \Delta}}\left(\left(\mathfrak{w}_{l}(x)\right)^{*} J \mathfrak{w}_{k}(x)+\left(\mathfrak{w}_{k}(x)\right)^{*} J \mathfrak{w}_{l}(x)\right)=0,
\end{aligned}
$$

which, together with (5.12), implies that the expression in (5.11) is equal to

$$
\sum_{l=0}^{\Delta} z^{l}\left(\mathfrak{w}_{l}(x)\right)^{*} J\left(f(x)-\lambda_{0} \sum_{k=\Delta+1}^{2 \Delta-l} z^{k} \mathfrak{w}_{k}(x)\right) .
$$

Since the integral in (5.9) exists, we can take the limit as $x \searrow \sigma_{1}$, which shows that

$$
\begin{aligned}
\left(\pi_{l} \circ \stackrel{\infty}{\Gamma}(F ; z F)\right)_{1}= & \lim _{x \searrow \sigma_{1}} \sum_{l=0}^{\Delta} z^{l}\left(\mathfrak{w}_{l}(x)\right)^{*} J\left(f(x)-\lambda_{0} \sum_{k=\Delta+1}^{2 \Delta-l} z^{k} \mathfrak{w}_{k}(x)\right) \\
& +\lambda_{0}\left(\sum_{l=1}^{2 \Delta} z^{l} d_{l-1}-\sum_{l=1}^{\ddot{o}} z^{2 \Delta+l} b_{\ddot{o}+1-l}\right) .
\end{aligned}
$$

Together with (2.21), the assertion of the lemma follows.

We also use the following consequence of Theorem 4.1.

5.5 Lemma. Let $\mathfrak{h}$ be a regular general Hamiltonian of the form 2.20. Let $z \in \mathbb{C}, F=(f ; \xi, \alpha) \in \stackrel{\infty}{\mathcal{P}}(\mathfrak{h})$, and assume that $(F ; z F) \in \stackrel{\infty}{T}(\mathfrak{h})$. Let $\mathfrak{w}_{l}$ and $\lambda_{l}$ be as in Lemma 5.4. Then

$$
\lambda_{0}=\lim _{x \searrow \sigma_{1}} f(x)_{2} .
$$

Proof. Let $G_{1}=\left(g_{1} ; \xi_{1}, \alpha_{1}\right), G_{2}=\left(g_{2} ; \xi_{2}, \alpha_{2}\right)$ be the unique elements with

$$
\begin{aligned}
& \left(G_{1} ; z G_{1}\right),\left(G_{2} ; z G_{2}\right) \in \stackrel{\infty}{T}(\mathfrak{h}), \\
& \pi_{l} \circ \stackrel{\infty}{\Gamma}\left(G_{1} ; z G_{1}\right)=\left(\begin{array}{l}
1 \\
0
\end{array}\right), \quad \pi_{l} \circ \stackrel{\infty}{\Gamma}\left(G_{2} ; z G_{2}\right)=\left(\begin{array}{l}
0 \\
1
\end{array}\right) .
\end{aligned}
$$

Then, by the definition of $\omega_{\mathfrak{h}}$ in $[\mathrm{KW} / \mathrm{V}]$, we have

$$
\omega_{\mathfrak{h}}(x ; z)=\left(\begin{array}{ll}
g_{1}(x)_{1} & g_{1}(x)_{2} \\
g_{2}(x)_{1} & g_{2}(x)_{2}
\end{array}\right) .
$$

By Theorem 4.1, thus

$$
\lim _{x \searrow \sigma_{1}} g_{1}(x)_{2}=0, \quad \lim _{x \searrow \sigma_{1}} g_{2}(x)_{2}=1 .
$$


If $F$ is any defect element, then $F$ can be written as a linear combination $F=a_{1} G_{1}+a_{2} G_{2}$. The numbers $a_{1}, a_{2}$ can be obtained by means of boundary values; in fact, we have

$$
a_{1}=\pi_{l, 1} \circ \stackrel{\infty}{\Gamma}(F ; z F), \quad a_{2}=\pi_{l, 2} \circ \stackrel{\infty}{\Gamma}(F ; z F) .
$$

Remembering (5.7) we obtain

$$
\lambda_{0}=\pi_{l, 2} \circ \stackrel{\infty}{\Gamma}(F ; z F)=a_{2}=\lim _{x \searrow_{\sigma_{1}}} f(x)_{2} .
$$

Proof (of Theorem 5.1 when $\alpha=\frac{\pi}{2}$ and $\left(\sigma_{1}, s_{1}\right)$ is not indivisible).

Let the general Hamiltonian $\mathfrak{h}$ be given according to the formulation of the theorem and let $z \in \mathbb{C}$ and $\psi$ be a solution of (5.1). Consider the general Hamiltonian $\mathfrak{h}_{\uparrow_{s}}$, i.e. the general Hamiltonian given by the data

$$
\sigma_{0}, \sigma_{1}, s_{1}, \quad H_{0},\left.H_{1}\right|_{\left(\sigma_{1}, s_{1}\right)}, \quad \ddot{o}, b_{j}, d_{j}, \quad E=\left\{\sigma_{0}, s_{1}\right\} .
$$

Then $\mathfrak{h}_{\text {} s_{1}}$ is of the form 2.20. Let $F=(f ; \xi, \alpha) \in \stackrel{\infty}{\mathcal{P}}\left(\mathfrak{h}_{\text {} s_{1}}\right)$ be the unique element such that $(F ; z F) \in \stackrel{\infty}{T}\left(\mathfrak{h}_{\uparrow s_{1}}\right)$ and $\pi_{r} \circ \stackrel{\infty}{\Gamma}\left(\mathfrak{h}_{\uparrow_{s}}\right)(F ; z F)=\psi\left(s_{1}\right)$. Denote the unique absolutely continuous representative of $f$ again by $f$. Then $f$ and $\psi$ are both absolutely continuous functions on $\left(\sigma_{1}, s_{1}\right]$ which satisfy the differential equation (5.1) and take the same boundary value at $s_{1}$. Thus

$$
\left.f\right|_{\left(\sigma_{1}, s_{1}\right]}=\left.\psi\right|_{\left(\sigma_{1}, s_{1}\right]}
$$

and hence $\stackrel{\infty}{\psi}(\mathfrak{h})(F)=\psi$, where $\stackrel{\infty}{\psi}(\mathfrak{h})$ is as in Remark 2.23. Lemmas 5.5 and 5.4 imply that the limits (5.2) and (5.3) exist and that

$$
\operatorname{rbv}(z) \psi=\pi_{l} \circ \stackrel{\infty}{\Gamma}\left(\mathfrak{h}_{\text {}_{1}}\right)(F ; z F),
$$

which shows (5.4).

Next let $a \in \mathbb{C}^{2}$ be given. There exists $(F ; z F) \in \stackrel{\infty}{T}\left(\mathfrak{h}_{\text {\} s _ { 1 } }) \text { with } \pi_{l} \text { 。 }\right.$ $\stackrel{\infty}{\Gamma}\left(\mathfrak{h}_{\uparrow_{1}}\right)(F ; z F)=a$. Write $F=(f ; \xi, \alpha)$; then $f$ is a solution of $(5.1)$ which has the required regularized boundary value. For uniqueness, let $z \in \mathbb{C}$ and assume that $\psi$ and $\tilde{\psi}$ are two solutions of (5.1) with

$$
\operatorname{rbv}(z) \psi=\operatorname{rbv}(z) \tilde{\psi}=: a .
$$

Since the defect element $(F ; z F) \in \stackrel{\infty}{T}\left(\mathfrak{h}_{\uparrow_{s}}\right)$ whose boundary value at $s_{1}$ equals $a$ is unique, it follows from (5.13) that $\psi=\tilde{\psi}$.

As usual, the proof for general values of $\alpha$ is carried out by applying rotation isomorphisms.

Proof (of Theorem 5.1, general values of $\alpha \in[0, \pi)$ ). Let $\mathfrak{h} \in \mathfrak{H}_{\alpha}, z \in \mathbb{C}$ and let $\psi$ be a solution of (5.1). The general Hamiltonian $\tilde{\mathfrak{h}}:=\circlearrowleft_{\alpha-\frac{\pi}{2}} \mathfrak{h}$ is in $\mathfrak{H}_{\frac{\pi}{2}}$. The function

$$
\tilde{\psi}(x):=N_{\alpha-\frac{\pi}{2}} \psi(x)
$$


is a solution of (5.1) with $H_{1}$ replaced by $\tilde{H}_{1}:=\circlearrowleft_{\alpha-\frac{\pi}{2}} H_{1}$.

We have

$$
\xi_{\alpha}^{T} \psi(x)=\xi_{\alpha}^{T} N_{\alpha-\frac{\pi}{2}}^{T} \cdot N_{\alpha-\frac{\pi}{2}} \psi(x)=\xi_{\frac{\pi}{2}}^{T} \tilde{\psi}(x),
$$

and hence $\operatorname{rbv}_{\mathrm{r}} \tilde{\psi}=\operatorname{rbv}_{\mathrm{r}} \psi$. Since $\tilde{\mathfrak{w}}_{l}=N_{\alpha-\frac{\pi}{2}} \mathfrak{w}_{l}$ and $N_{\alpha-\frac{\pi}{2}}^{*} J N_{\alpha-\frac{\pi}{2}}=J$, we also have $\operatorname{rbv}_{\mathrm{s}}(z) \tilde{\psi}=\operatorname{rbv}_{\mathrm{s}}(z) \psi$.

The fact that $\xi_{\alpha}$ and $\xi_{\alpha-\frac{\pi}{2}}$ are linearly independent implies that, for each given $a \in \mathbb{C}^{2}$, there exists a unique solution $\psi$ with $\operatorname{rbv}(z) \psi=a$.

Finally, it follows from (5.14) that

$$
\pi_{l} \circ \tilde{\tilde{\Phi}}(\tilde{F} ; z \tilde{F})=\operatorname{rbv}(z) \tilde{\psi}=\operatorname{rbv}_{\mathrm{s}}(z) \tilde{\psi} \cdot \xi_{0}+\operatorname{rbv}_{\mathrm{r}} \tilde{\psi} \cdot \xi_{\frac{\pi}{2}} .
$$

Moreover,

$$
\pi_{l} \circ \tilde{\tilde{\Phi}}(F ; z F)=N_{\frac{\pi}{2}-\alpha}\left[\pi_{l} \circ \tilde{\tilde{\Phi}}(\tilde{F} ; z \tilde{F})\right]=\operatorname{rbv}_{\mathrm{s}}(z) \tilde{\psi} \cdot \xi_{\alpha-\frac{\pi}{2}}+\operatorname{rbv}_{\mathrm{r}} \tilde{\psi} \cdot \xi_{\alpha}
$$

and hence

$$
\pi_{l} \circ \stackrel{\infty}{\Gamma}(F ; z F)=\operatorname{rbv}(z) \psi .
$$

Observing Remark 2.23 we obtain (5.4).

We come to the proof of Theorem 5.2. It relies mainly on Theorem 4.1.

Proof (of Theorem 5.2). By the usual rotation argument, we can restrict the explicit proof to the case when $\alpha=\frac{\pi}{2}$.

Let $z \in \mathbb{C}$ and let a solution $\psi$ be given. Set

$$
\psi_{\mathrm{r}}(x ; z):=\left[(1,0) \omega_{\mathfrak{h}}(x ; z)\right]^{T}, \quad \psi_{\mathrm{s}}(x ; z):=\left[(0,1) \omega_{\mathfrak{h}}(x ; z)\right]^{T}, \quad x \in\left(\sigma_{1}, \sigma_{2}\right) .
$$

Then $\psi_{\mathrm{r}}(\cdot ; z)$ and $\psi_{\mathrm{s}}(\cdot ; z)$ are linearly independent solutions of $(5.1)$, and thus each given solution $\psi$ can be written as a linear combination $\psi=a_{1} \psi_{\mathrm{r}}(\cdot ; z)+$ $a_{2} \psi_{\mathrm{s}}(\cdot ; z)$. As we have already noted in the proof of Lemma 5.5,

$$
\operatorname{rbv}(z) \psi=\left(\begin{array}{c}
a_{1} \\
a_{2}
\end{array}\right) .
$$

By Theorem 4.1 we have

$$
\lim _{x \searrow \sigma_{1}} \psi_{\mathrm{r}}(x ; z)=\left(\begin{array}{l}
1 \\
0
\end{array}\right), \quad \lim _{x \searrow \sigma_{1}} \psi_{\mathrm{s}}(x ; z)_{2}=1
$$

and

$$
\lim _{x \searrow \sigma_{1}} \psi_{\mathrm{s}}(x ; z)_{1}=\infty \quad \text { if } z \neq 0,
$$

which implies (5.5). Moreover, we see that the equivalence '( $i) \Leftrightarrow(i i)$ ' holds true since both of $(i)$ and $(i i)$ are equivalent to ' $a_{2}=0$ '. In this case we also have $\left(\operatorname{rbv}(z) \psi_{1}=\lim _{x \searrow \sigma_{1}}(\psi(x))_{1}\right.$, which, together with (5.5), is (5.6). The equivalence $(i i) \Leftrightarrow(i i i)$ is trivial. 
In order to establish the equivalence with $(i v)$, we have to assume that $z \neq 0$ and we distinguish the cases when $z$ is real and when it is non-real. Assume first that $z \notin \mathbb{R}$. Green's identity (2.11) applied on the interval $\left(x, s_{1}\right)$ gives

$$
2 i \operatorname{Im} z \int_{x}^{s_{1}} \psi(t)^{*} H(t) \psi(t) d t=\psi(x)^{*} J \psi(x)-\psi\left(s_{1}\right)^{*} J \psi\left(s_{1}\right) .
$$

Hence the implication ' $(i) \Rightarrow(i v)$ ' holds, and we conclude that $\psi_{\mathrm{r}}(\cdot ; z)$ satisfies (iv). However, any two solutions of (5.1) that satisfy (iv) are linearly dependent. Thus each solution with $(i v)$ must be a scalar multiple of $\psi_{\mathrm{r}}(\cdot ; z)$, and hence satisfies $(i)$. We therefore see that also the converse implication ' $(i v) \Rightarrow(i)$ ' holds.

Consider now the case that $z \in \mathbb{R}$. Assume that $\psi \in L^{2}\left(\left.H\right|_{\left(\sigma_{1}, s_{1}\right)}\right)$. Since $\left.H\right|_{\left(\sigma_{1}, s_{1}\right)}$ satisfies (HS-), the minimal operator in $L^{2}\left(\left.H\right|_{\left(\sigma_{1}, s_{1}\right)}\right)$ is entire. Hence we can choose a family $\psi_{w} \in L^{2}\left(\left.H\right|_{\left(\sigma_{1}, s_{1}\right)}\right)$ which is defined and analytic in some open neighbourhood $U$ of $z$ and solves the equation (5.1) with $z$ replaced by $w$. Let $a_{1}(w)$ and $a_{2}(w)$ be the unique functions such that

$$
\psi_{w}=a_{1}(w) \psi_{\mathrm{r}}(\cdot ; w)+a_{2}(w) \psi_{\mathrm{s}}(\cdot ; w) .
$$

Comparing boundary values at $s_{1}$ we obtain that

$$
\psi_{w}\left(s_{1}\right)=a_{1}(w) \psi_{\mathrm{r}}\left(s_{1} ; w\right)+a_{2}(w) \psi_{\mathrm{s}}\left(s_{1} ; w\right) .
$$

Since the boundary map is continuous, $\psi_{w}\left(s_{1}\right)$ depends analytically on $w$. As $\psi_{\mathrm{r}}$ and $\psi_{\mathrm{s}}$ are linearly independent, this implies that also $a_{1}(w)$ and $a_{2}(w)$ are analytic functions. By the already proved case ' $z \notin \mathbb{R}$ ', we have $a_{2}(w)=0$ for $w \in U \backslash \mathbb{R}$. Thus also $a_{2}(w)=0$ for $w \in U \cap \mathbb{R}$ and therefore $\psi$ is a scalar multiple of $\psi_{\mathrm{r}}(\cdot ; z)$, which implies that $\psi$ satisfies $(i)$. Hence the implication $(i v) \Rightarrow(i)$ holds. For the converse remember that the minimal operator in $L^{2}\left(\left.H\right|_{\left(\sigma_{1}, s_{1}\right)}\right)$ has deficiency index $(1,1)$ and is an entire operator. Therefore we know that there exists a non-trivial solution in $L^{2}\left(\left.H\right|_{\left(\sigma_{1}, s_{1}\right)}\right)$. By the above considerations, we know that such a solution must be a scalar multiple of $\psi_{\mathrm{r}}(\cdot ; z)$, which implies that $\psi_{\mathrm{r}}(\cdot ; z) \in L^{2}\left(\left.H\right|_{\left(\sigma_{1}, s_{1}\right)}\right)$. This proves the implication $(i) \Rightarrow(i v)$ also in the case when $z \in \mathbb{R}$.

5.6 Remark. Let $\mathfrak{h}$ be as in Theorem 5.1 and $\tilde{\mathfrak{h}}$ a reparameterization of $\mathfrak{h}$ in the sense of [KW/V, Remark 3.38]. Then $\tilde{\mathfrak{h}}$ again belongs to $\mathfrak{H}_{\alpha}$ by Theorem 3.1, and there exists an isomorphism of the form $(\varpi, \mathrm{id} \times \mathrm{id})$ of the corresponding boundary triples by $[\mathrm{KW} / \mathrm{V}$, Remark 3.39]. Denote by $\widetilde{\operatorname{rbv}}(z)$ the generalized boundary value as in Theorem 5.1 for $\tilde{\mathfrak{h}}$. Then $\widetilde{\operatorname{rbv}}(z) \tilde{\psi}=\operatorname{rbv}(z) \psi$ whenever $\psi=\psi(\mathfrak{h}) F, \tilde{\psi}=\psi(\tilde{\mathfrak{h}}) \tilde{F}$ and $\varpi F=\tilde{F}$.

As a corollary we obtain a construction of the fundamental solution and the Titchmarsh-Weyl coefficient that is exactly analogous to the classical (positive definite) case.

5.7 Corollary (Computation of the fundamental solution). Let $\mathfrak{h}$ be a singular general Hamiltonian as in Theorem 5.1 and let

$$
\theta(x ; z)=\left(\theta_{1}(x ; z), \theta_{2}(x ; z)\right)^{T}, \quad \varphi(x ; z)=\left(\varphi_{1}(x ; z), \varphi_{2}(x ; z)\right)^{T}
$$


be the unique solutions of (5.1) with

$$
\operatorname{rbv}(z) \theta(\cdot ; z)=\left(\begin{array}{l}
1 \\
0
\end{array}\right), \quad \operatorname{rbv}(z) \varphi(\cdot ; z)=\left(\begin{array}{l}
0 \\
1
\end{array}\right)
$$

Then

$$
\omega_{\mathfrak{h}}(x ; z)=\left(\begin{array}{ll}
\theta_{1}(x ; z) & \theta_{2}(x ; z) \\
\varphi_{1}(x ; z) & \varphi_{2}(x ; z)
\end{array}\right), \quad x \in\left(\sigma_{1}, \sigma_{2}\right) .
$$

The Titchmarsh-Weyl coefficient $q_{\mathfrak{h}}$ can be obtained as the limit (which is independent of $\tau \in \mathbb{R} \cup\{\infty\}$ )

$$
q_{\mathfrak{h}}(z)=\lim _{x \nearrow \sigma_{2}} \frac{\theta_{1}(x ; z) \tau+\theta_{2}(x ; z)}{\varphi_{1}(x ; z) \tau+\varphi_{2}(x ; z)}
$$

or as the unique function with

$$
\theta(\cdot ; z)-\left.q_{\mathfrak{h}}(z) \varphi(\cdot ; z)\right|_{\left(s_{1}, \sigma_{2}\right)} \in L^{2}\left(\left.H_{1}\right|_{\left(s_{1}, \sigma_{2}\right)}\right),
$$

where $s_{1} \in\left(\sigma_{1}, \sigma_{2}\right)$.

Proof. For a solution $\psi$ of $(5.1)$ the vector $\operatorname{rbv}(z) \psi$ is exactly the boundary value at $\sigma_{0}$ of the defect element $(F ; z F)$ with $\left.f\right|_{\left(\sigma_{1}, \sigma_{2}\right)}=\psi($ where $F=(f ; \xi, \alpha))$. Hence the asserted formula for $\omega_{\mathfrak{h}}$ is merely its definition. The statements about the Titchmarsh-Weyl coefficient are immediate.

5.8 Remark. Viewing the above formulae from a slightly different point, leads to the following way to compute $\omega_{\mathfrak{h}}$ which may sometimes be more practical. Let $x \in\left(\sigma_{1}, \sigma_{2}\right)$, and let $\hat{\theta}(\cdot ; z)$ and $\hat{\varphi}(\cdot ; z)$ be the solutions of $(5.1)$ with

$$
\hat{\theta}(x ; z)=\left(\begin{array}{l}
1 \\
0
\end{array}\right), \quad \hat{\varphi}(x ; z)=\left(\begin{array}{l}
0 \\
1
\end{array}\right) .
$$

Then

$$
\omega_{\mathfrak{h}}(x ; z)^{T}=(\operatorname{rbv}(z) \hat{\theta} ; \operatorname{rbv}(z) \hat{\varphi})^{-1}
$$

We close this section with answering the question how the Titchmarsh-Weyl coefficient of a general Hamiltonian $\mathfrak{h} \in \mathfrak{H}_{\alpha}$ transforms when the data part ' $\ddot{o}, b_{j}, d_{j}$ ' of $\mathfrak{h}$ is altered but the Hamiltonian function $H_{1}$ is kept fixed. This generalizes the case ' $\left(\sigma_{0}, \sigma_{1}\right)$ indivisible' of a previous result in [LW1] to higher negative indices. In [LW1, Theorem 5.4] we have answered the corresponding question for general Hamiltonians with ind $\mathfrak{h}=1$ (not necessarily satisfying $\left.\left(\mathrm{gH}_{\alpha}\right)\right)$. However, the case when $\left(\sigma_{0}, \sigma_{1}\right)$ is indivisible already there played a special role, cf. [LW1, Corollary 5.5].

For simplicity, we restrict our attention to the case that $\alpha=0$. As usual, the corresponding versions for other values of $\alpha \in[0, \pi)$ can be deduced by applying rotation isomorphisms. Note that in [LW1, Corollary 5.5] the case $\alpha=\frac{\pi}{2}$ was considered; in this situation one has to replace $q_{\mathfrak{h}}$ by $-\frac{1}{q_{\mathfrak{h}}}$ and $q_{\mathfrak{h}_{0}}$ by $-\frac{1}{q_{\mathfrak{h}_{0}}}$ in the corollary below. 
5.9 Corollary. Let $\mathfrak{h}$ be a general Hamiltonian $\mathfrak{h} \in \mathfrak{H}_{0}$ which is given by the data

$$
\sigma_{0}, \sigma_{1}, \sigma_{2}, \quad H_{0}, H_{1}, \quad \ddot{o}, b_{j}, d_{j}, \quad E,
$$

where $b_{\ddot{o}+1}=0$ when $\sigma_{1}$ is not left endpoint of an indivisible interval, and denote by $\mathfrak{h}_{0}$ the general Hamiltonian given by

$$
\sigma_{0}, \sigma_{1}, \sigma_{2}, \quad H_{0}, H_{1}, \quad \ddot{o}_{0}:=0, b_{0,1}:=0, d_{0, j}:=0, \quad E .
$$

Then

$$
q_{\mathfrak{h}}(z)=q_{\mathfrak{h}_{0}}(z)+\sum_{l=1}^{2 \Delta} z^{l} d_{l-1}-\sum_{l=1}^{\ddot{o}} z^{2 \Delta+l} b_{\ddot{o}+1-l} .
$$

Proof. We use Corollary 5.7 to compute $\omega_{\mathfrak{h}}$ and $\omega_{\mathfrak{h}_{0}}$. Let $\varphi(\cdot ; z)$ and $\theta(\cdot ; z)$ be the solutions of (5.1) whose regularized boundary values with respect to the general Hamiltonian $\mathfrak{h}$ are equal to

$$
\operatorname{rbv}^{\mathfrak{h}}(z) \theta(\cdot ; z)=\left(\begin{array}{l}
1 \\
0
\end{array}\right), \quad \operatorname{rbv}^{\mathfrak{h}}(z) \varphi(\cdot ; z)=\left(\begin{array}{l}
0 \\
1
\end{array}\right)
$$

i.e.

$$
\begin{aligned}
& \operatorname{rbv}_{\mathrm{r}}^{\mathfrak{h}} \theta(\cdot ; z)=1, \operatorname{rbv}_{\mathrm{s}}^{\mathfrak{h}}(z) \theta(\cdot ; z)=0, \\
& \operatorname{rbv}_{\mathrm{r}}^{\mathfrak{h}} \varphi(\cdot ; z)=0, \quad \operatorname{rbv}_{\mathrm{s}}^{\mathfrak{h}}(z) \varphi(\cdot ; z)=-1 .
\end{aligned}
$$

Since $\mathrm{rbv}_{\mathrm{r}}$ is given as a limit of the function itself (the data $\ddot{o}, b_{j}, d_{j}$ do not enter the formula), we have

$$
\operatorname{rbv}_{\mathrm{r}}^{\mathfrak{h}_{0}} \varphi(\cdot ; z)=\operatorname{rbv}_{\mathrm{r}}^{\mathfrak{h}} \varphi(\cdot ; z)=0, \quad \operatorname{rbv}_{\mathrm{r}}^{\mathfrak{h}_{0}} \theta(\cdot ; z)=\operatorname{rbv}_{\mathrm{r}}^{\mathfrak{h}} \theta(\cdot ; z)=1 .
$$

The functions $\mathfrak{w}_{l}$ also do not depend on the data $\ddot{o}, b_{j}, d_{j}$, which implies that

$$
\operatorname{rbv}_{\mathrm{S}}^{\mathfrak{h}}(z) \varphi(\cdot ; z)=\lim _{x \searrow \sigma_{1}}\left[\sum_{l=0}^{\Delta} z^{l}\left(\mathfrak{w}_{l}(x)\right)^{*} J \varphi(x ; z)\right]=\operatorname{rbv}_{\mathrm{S}}^{\mathfrak{h}_{0}}(z) \varphi(\cdot ; z) .
$$

Set

$$
p(x):=\sum_{l=1}^{2 \Delta} z^{l} d_{l-1}-\sum_{l=1}^{\ddot{o}} z^{2 \Delta+l} b_{\ddot{o}+1-l}
$$

and

$$
\varphi_{0}(\cdot ; z):=\varphi(\cdot ; z), \quad \theta_{0}(\cdot ; z):=\theta(\cdot ; z)-p(z) \varphi(\cdot ; z)
$$

Then

$$
\begin{aligned}
\operatorname{rbv}^{\mathfrak{h}_{0}}(z) \varphi_{0}(\cdot ; z) & =\operatorname{rbv}^{\mathfrak{h}_{0}}(z) \varphi(\cdot ; z)=\operatorname{rbv}^{\mathfrak{h}}(z) \varphi(\cdot ; z)=\left(\begin{array}{l}
0 \\
1
\end{array}\right), \\
\operatorname{rbv}_{\mathrm{r}}^{\mathfrak{h}_{0}} \theta_{0}(\cdot ; z) & =\operatorname{rbv}_{\mathrm{r}}^{\mathfrak{h}} \theta_{0}(\cdot ; z)=\operatorname{rbv}_{\mathrm{r}}^{\mathfrak{h}} \theta(\cdot ; z)-p(z) \operatorname{rbv}_{\mathrm{r}}^{\mathfrak{h}} \varphi(\cdot ; z)=1
\end{aligned}
$$

and

$$
\begin{aligned}
\operatorname{rbv}_{\mathbf{s}}^{\mathfrak{h}_{0}}(z) \theta_{0}(\cdot ; z) & =\operatorname{rbv}_{\mathbf{s}}^{\mathfrak{h}_{0}}(z) \theta(\cdot ; z)-p(z) \operatorname{rbv}_{\mathbf{s}}^{\mathfrak{h}_{0}}(z) \varphi(\cdot ; z) \\
& =\lim _{x \searrow \sigma_{1}}\left[\sum_{l=0}^{\Delta} z^{l}\left(\mathfrak{w}_{l}(x)\right)^{*} J\left(\theta(x ; z)-\sum_{k=\Delta+1}^{2 \Delta-l} z^{k} \mathfrak{w}_{k}(x)\right)\right]+p(z) \\
& =\operatorname{rbv}_{\mathbf{S}}^{\mathfrak{h}}(z) \theta(\cdot ; z)=0
\end{aligned}
$$


i.e. $\operatorname{rbv}^{\mathfrak{h}_{0}}(z) \theta_{0}(\cdot ; z)=\left(\begin{array}{l}1 \\ 0\end{array}\right)$. Hence the fundamental solutions $\omega_{\mathfrak{h}}$ and $\omega_{\mathfrak{h}_{0}}$ are given by

$$
\begin{aligned}
\omega_{\mathfrak{h}}(x ; z) & =\left(\begin{array}{cc}
\theta_{1}(x ; z) & \theta_{2}(x ; z) \\
\varphi_{1}(x ; z) & \varphi_{2}(x ; z)
\end{array}\right), \\
\omega_{\mathfrak{h}_{0}}(x ; z) & =\left(\begin{array}{cc}
\theta_{1}(x ; z)-p(z) \varphi_{1}(x ; z) & \theta_{2}(x ; z)-p(z) \varphi_{2}(x ; z) \\
\varphi_{1}(x ; z) & \varphi_{2}(x ; z)
\end{array}\right) .
\end{aligned}
$$

It follows that

$$
\begin{aligned}
q_{\mathfrak{h}_{0}}(z) & =\lim _{x \nearrow \sigma_{2}} \omega_{\mathfrak{h}_{0}}(x ; z) \star \infty=\lim _{x \nearrow \sigma_{2}} \frac{\theta_{1}(x ; z)-p(z) \varphi_{1}(x ; z)}{\varphi_{1}(x ; z)} \\
& =\lim _{x \nearrow \sigma_{2}} \frac{\theta_{1}(x ; z)}{\varphi_{1}(x ; z)}-p(z)=q_{\mathfrak{h}}(z)-p(z),
\end{aligned}
$$

which implies the asserted formula.

\section{Acknowledgements}

The first author (M.L.) gratefully acknowledges the support of the Engineering and Physical Sciences Research Council (EPSRC), grant no. EP/E037844/1. Both authors thank the Isaac Newton Institute for Mathematical Sciences, Cambridge, for its hospitality.

\section{References}

[ADSR] D. Alpay, A. DiJksma, H.S.V. DE Snoo, J. Rovnyak: Schur Functions, Operator Colligations, and Reproducing Kernel Pontryagin Spaces, Oper. Theory Adv. Appl. 96, Birkhäuser Verlag, Basel 1997.

[dB] L. DE Branges: Hilbert Spaces of Entire Functions, Prentice-Hall, London 1968.

[DHS] V.Derkach, S. Hassi, H. DE SNoo: Asymptotic expansions of generalized Nevanlinna functions and their spectral properties, Oper. Theory Adv. Appl. 175 (2007), 51-88.

[DKuS] A. Dijksma, P. Kurasov, Yu. Shondin: High order singular rank one perturbations of a positive operator, Integral Equations Operator Theory 53 (2005), 209-245.

[DLlS] A. Dijksma, H. Langer, A. Luger, Yu. Shondin: A factorization result for generalized Nevanlinna functions of the class $\mathcal{N}_{\kappa}$, Integral Equations Operator Theory 36 (2000), 121-125.

[DLS] A. Dijksma, H. LAnger, Yu. Shondin: Rank one perturbations at infinite coupling in Pontryagin spaces, J. Funct. Anal. 209 (2004), 206-246.

[DLSZ] A. Dijksma, H. Langer, Yu. Shondin, C. Zeinstra: Self-adjoint operators with inner singularities and Pontryagin spaces, Oper. Theory Adv. Appl. 118 (2000), 105-175.

[DLuS1] A. Dijksma, A. Luger, Yu. Shondin: Minimal models for $\mathcal{N}_{\kappa}^{\infty}$-functions, Oper. Theory Adv. Appl. 163 (2006), 97-134. 
[DLuS2] A. Dijksma, A. Luger, Yu. Shondin: Approximation of $\mathcal{N}_{\kappa}^{\infty}$-functions. $I$. Models and regularization, Oper. Theory Adv. Appl. 188 (2009), 87-112.

[DLuS3] A. DiJksma, A. Luger, Yu. Shondin: Approximation of $\mathcal{N}_{\kappa}^{\infty}$-functions. II. Convergence of models, Oper. Theory Adv. Appl. 198 (2010), 125-169.

[HSW] S. HASSI, H. DE SNOO, H. WINKLER: Boundary-value problems for two-dimensional canonical systems, Integral Equations Operator Theory 36 (2000), 445479.

[HL] S. HAssi, A. Luger: Generalized zeros and poles of $\mathcal{N}_{\kappa}$ functions: on the underlying spectral structure, Methods Funct. Anal. Topology 12 (2006), 131150 .

[H] G. Herglotz: Über Potenzreihen mit positivem, reellen Teil im Einheitskreis, Berichte ü.d. Verhandlungen d. Königlich Sächsischen Gesellschaft d. Wiss. zu Leipzig. Math.-phys. Klasse 63 (1911), 501-511.

[K] I. S. KAC: On the Hilbert spaces generated by monotone Hermitian matrix functions (Russian), Kharkov, Zap. Mat.o-va, 22 (1950), 95-113.

[KW/I] M. Kaltenbäck, H. Woracek: Pontryagin spaces of entire functions I, Integral Equations Operator Theory 33 (1999), 34-97.

[KW/II] M. Kaltenbäck, H. WoraceK: Pontryagin spaces of entire functions II, Integral Equations Operator Theory 33 (1999), 305-380.

[KW/III] M. Kaltenbëck, H. WoraceK: Pontryagin spaces of entire functions III, Acta Sci.Math. (Szeged) 69 (2003), 241-310.

[KW/IV] M. Kaltenbëck, H. WoraceK: Pontryagin spaces of entire functions $I V$, Acta Sci. Math. (Szeged) 72 (2006), 709-835.

[KW/V] M. Kaltenbëck, H. Woracek: Pontryagin spaces of entire functions $V$, Acta Sci. Math. (Szeged) 77 (2011), 223-336.

[KW/VI] M. Kaltenbëck, H. WoraceK: Pontryagin spaces of entire functions VI, Acta Sci. Math. (Szeged) 76 (2010), 511-560.

[KL1] M. G. KRĔ̌N, H. LANGER: Über die Q-Funktion eines П-hermiteschen Operators im Raume $\Pi_{\kappa}$, Acta Sci. Math. (Szeged) 34 (1973), 191-230.

[KL2] M. G. KreǏn, H. LANGER: Über einige Fortsetzungsprobleme, die eng mit der Theorie hermitescher Operatoren im Raume $\Pi_{\kappa}$ zusammenhängen. I. Einige Funktionenklassen und ihre Darstellungen, Math. Nachr. 77 (1977), 187-236.

[KL3] M. G. KREIN, H. LANGER: Some propositions on analytic matrix functions related to the theory of operators in the space $\Pi_{\kappa}$, Acta Sci. Math. (Szeged) 43 (1981), 181-205.

[KuLu] P. Kurasov, A. Luger: An operator theoretic interpretation of the generalized Titchmarsh-Weyl coefficient for a singular Sturm-Liouville problem, Math. Phys. Anal. Geom. 14 (2011), 115-151.

[L] H. LANGER: A characterization of generalized zeros of negative type of functions of the class $\mathcal{N}_{\kappa}$, Oper. Theory Adv. Appl. 17 (1986), 201-212.

[LW1] M. LAnger, H. WoraceK: Dependence of the Weyl coefficient on singular interface conditions, Proc. Edinburgh Math. Soc. 52 (2009), 445-487.

[LW2] M. LANGER, H. WoraceK: A function space model for canonical systems with an inner singularity, Acta Sci. Math. (Szeged) 77 (2011), 101-165.

[LW3] M. LAnger, H. WoraceK: Direct and inverse spectral theorems for a class of Hamiltonian systems with two singular endpoints, in preparation.

[Le] B. JA Levin: Distribution of Zeros of Entire Functions, American Mathematical Society, Providence, R.I. 1964. 
[P] G. PICK: Über die Beschränkungen analytischer Funktionen, welche durch vorgegebene Funktionswerte bewirkt werden, Math. Ann. 77 (1916), 7-23.

[RR94] M. Rosenblum, J. RovnyaK: Topics in Hardy Classes and Univalent Functions, Birkhäuser Advanced Texts: Basler Lehrbücher, Birkhäuser Verlag, Basel, 1994.

[Wi1] H. WinkLER: The inverse spectral problem for canonical systems, Integral Equations Operator Theory 22 (1995), 360-374.

[Wi2] H. WinkLeR: Canonical systems with a semibounded spectrum, Oper. Theory Adv. Appl. 106 (1998), 397-417.

[WW] H. Winkler, H. WORACEK: On semibounded canonical systems, Lin. Alg. Appl. 429 (2008), 1082-1092.

[Wo] H. WorACEK: Existence of zerofree functions $N$-associated to a de Branges Pontryagin space, Monatsh. Math. 162 (2011), 453-506.

M. Langer

Department of Mathematics and Statistics

University of Strathclyde

26 Richmond Street

Glasgow G1 1XH

UNITED KINGDOM

email: m.langer@strath.ac.uk

H. Woracek

Institut für Analysis und Scientific Computing

Technische Universität Wien

Wiedner Hauptstr. 8-10/101

A-1040 Wien

AUSTRIA

email: harald.woracek@tuwien.ac.at 